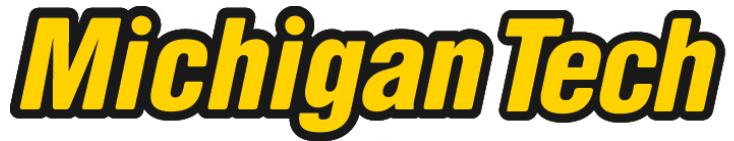 \\ Michigan Technological University Create the Future Digital Commons @ Michigan Tech
}

2011

\section{Multi-software modeling technique for field distribution propagation through an optical vertical interconnect assembly}

Matthew D. Howard

Michigan Technological University

Follow this and additional works at: https://digitalcommons.mtu.edu/etds

Part of the Electrical and Computer Engineering Commons

Copyright 2011 Matthew D. Howard

\section{Recommended Citation}

Howard, Matthew D., "Multi-software modeling technique for field distribution propagation through an optical vertical interconnect assembly", Master's Thesis, Michigan Technological University, 2011.

https://doi.org/10.37099/mtu.dc.etds/47

Follow this and additional works at: https://digitalcommons.mtu.edu/etds

Part of the Electrical and Computer Engineering Commons 
A MULTI-SOFTWARE MODELING TECHNIQUE FOR FIELD DISTRIBUTION PROPAGATION THROUGH AN OPTICAL VERTICAL INTERCONNECT ASSEMBLY

by

Matthew D. Howard

\begin{abstract}
A THESIS
Submitted in partial fulfillment of the requirements for the degree of

MASTER OF SCIENCE

(Electrical Engineering)
\end{abstract}

MICHIGAN TECHNOLOGICAL UNIVERSITY

2011

(C)2011 Matthew D. Howard 

This thesis, "A Multi-Software Modeling Technique for Field Distribution Propagation through an Optical Vertical Interconnect Assembly," is hereby approved in partial fulfillment of the requirements for the degree MASTER OF SCIENCE IN ELECTRICAL ENGINEERING.

Department of Electrical and Computer Engineering

Signatures:

Thesis Advisor

Dr. Christopher T. Middlebrook

Committee Member

Dr. Michael C. Roggemann

Committee Member

Dr. Warren F. Perger

Committee Member

Dr. Craig R. Friedrich

Department Chair

Dr. Daniel R. Fuhrmann

Date 



\section{Table of Contents}

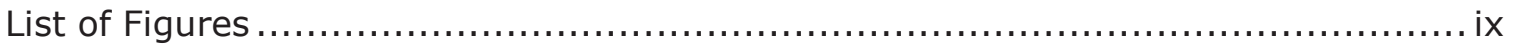

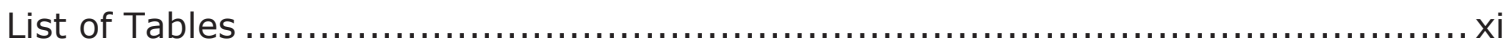

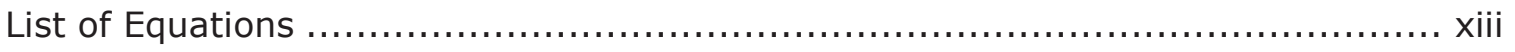

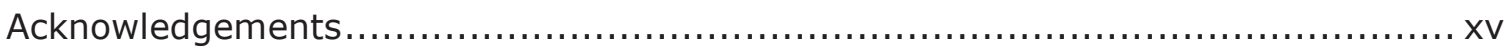

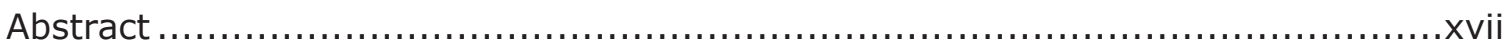

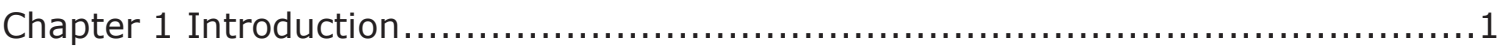

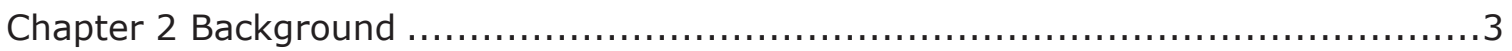

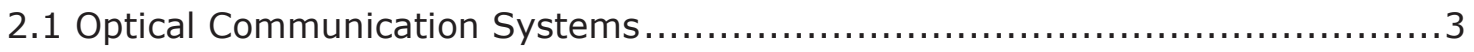

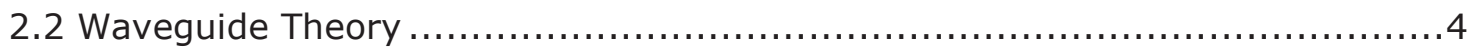

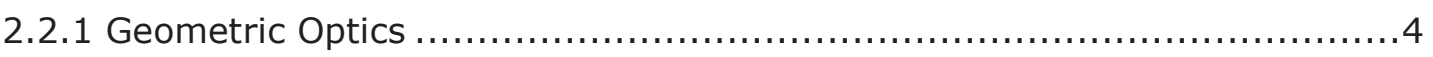

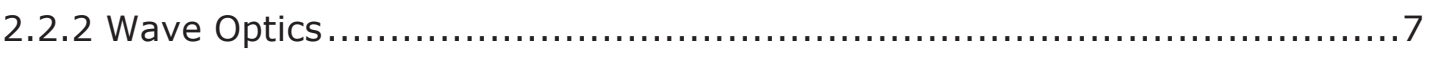

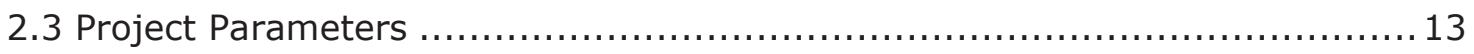

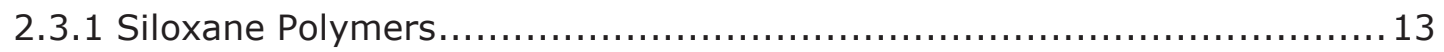

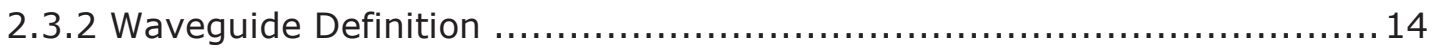

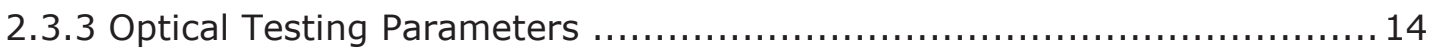

2.4 Vertical Interconnect Assembly .................................................... 15

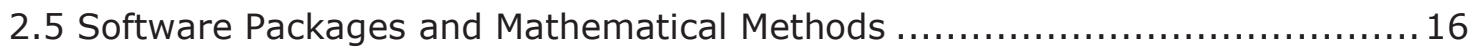

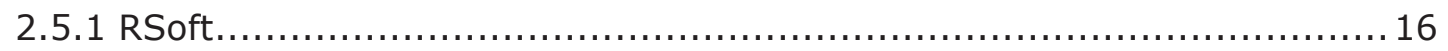

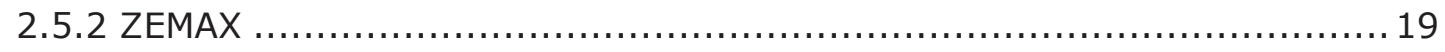

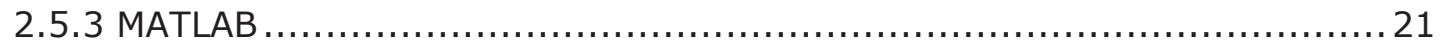

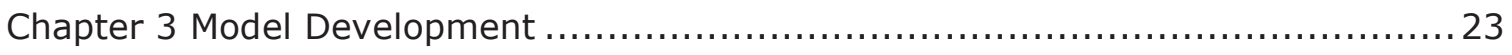

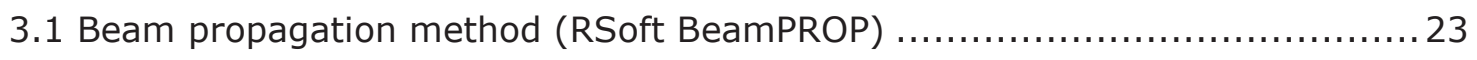


3.2 Finite difference time domain method (RSoft FullWAVE) $\ldots \ldots \ldots \ldots \ldots \ldots \ldots \ldots \ldots \ldots \ldots \ldots \ldots \ldots \ldots$

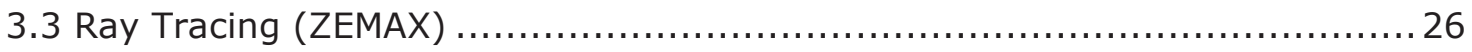

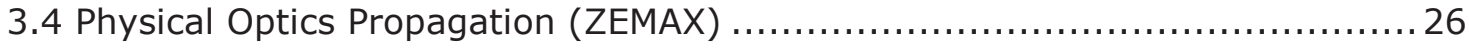

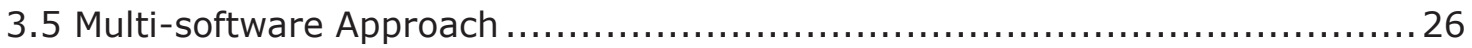

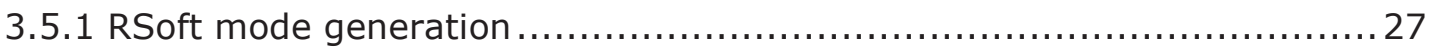

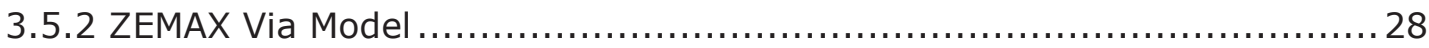

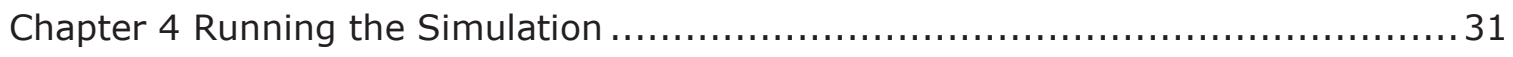

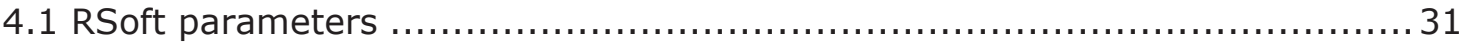

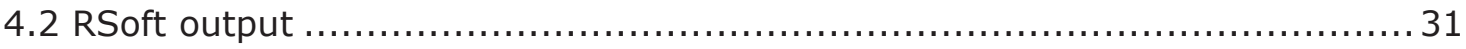

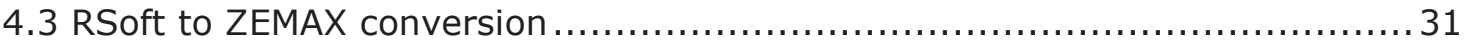

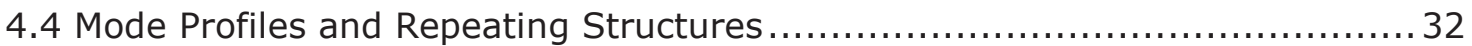

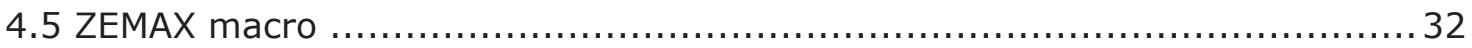

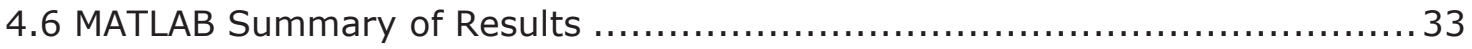

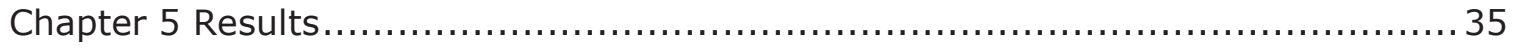

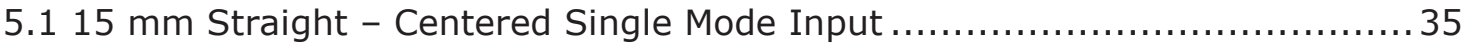

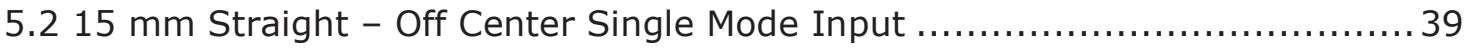

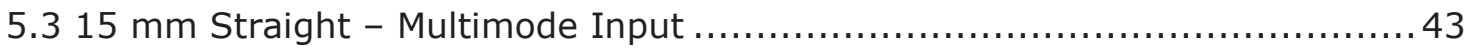

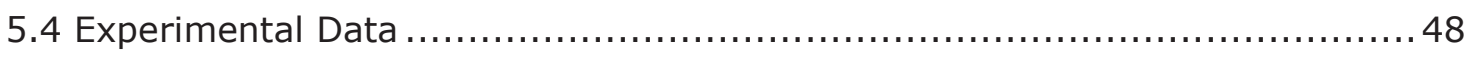

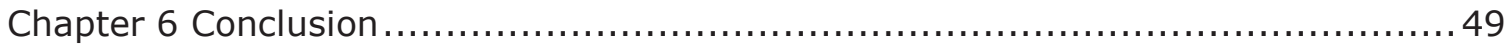

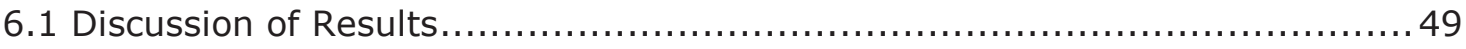

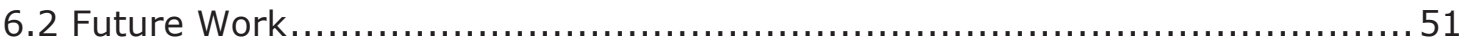

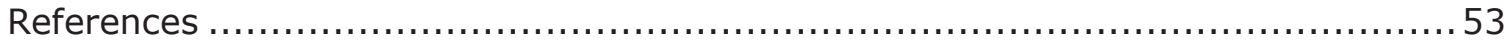

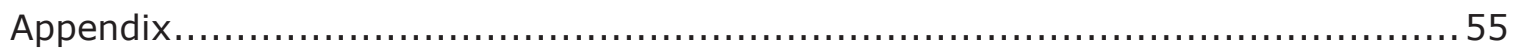

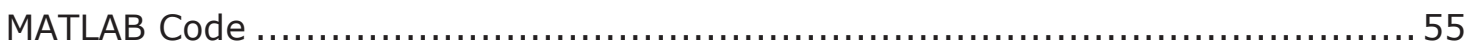




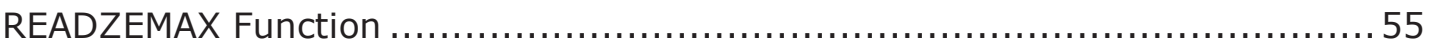

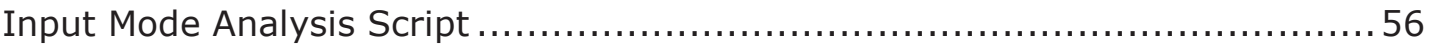

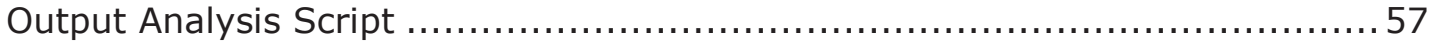

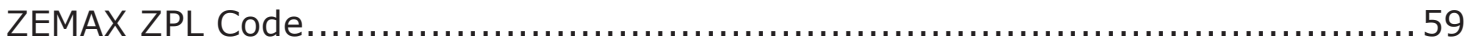

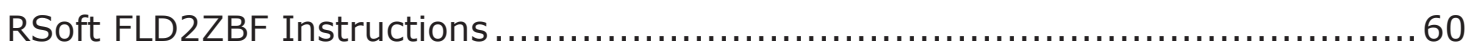





\section{List of Figures}

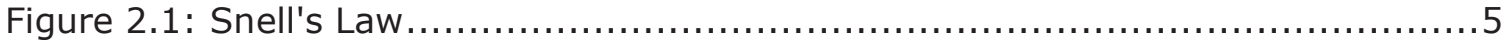

Figure 2.2: Critical angle by Snell's Law and Fresnel Coefficients....................6

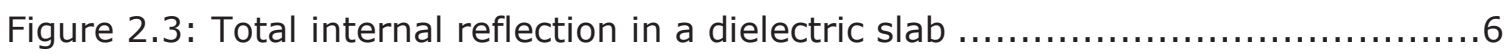

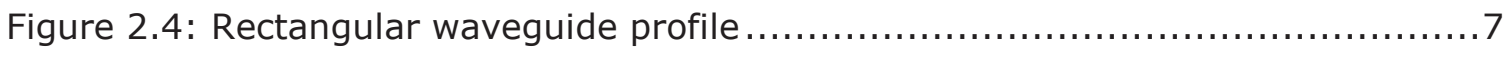

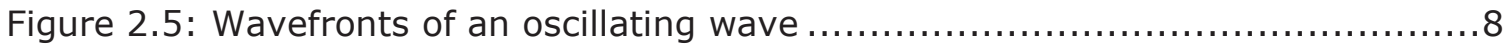

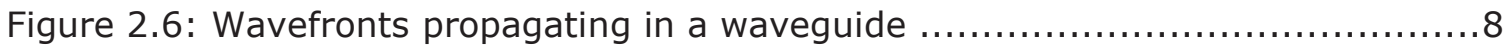

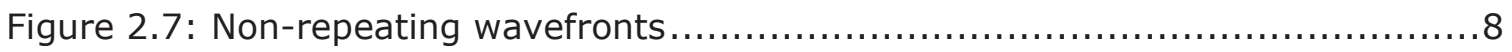

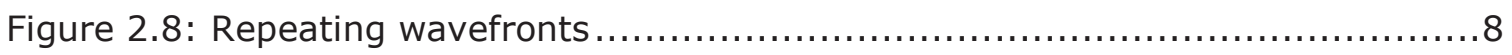

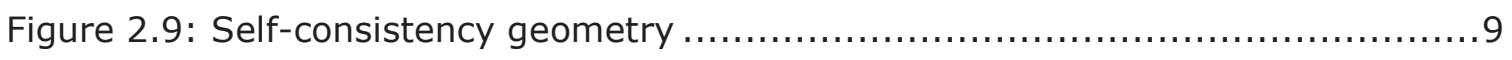

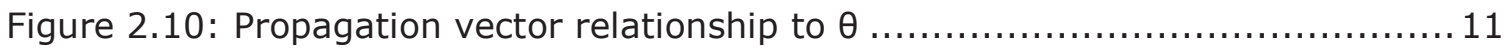

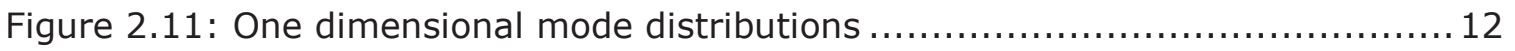

Figure 2.12: Normalized two dimensional mode field distributions $\ldots \ldots \ldots \ldots \ldots \ldots \ldots \ldots$

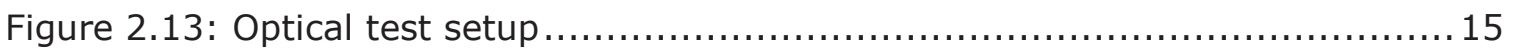

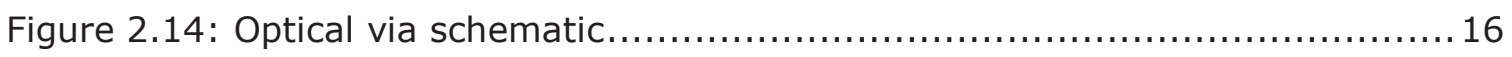

Figure 2.15: Propagation method selection based on Fresnel number ................ 20

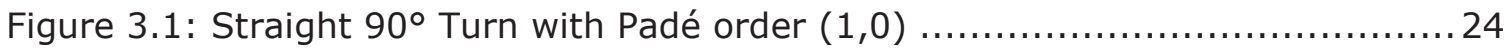

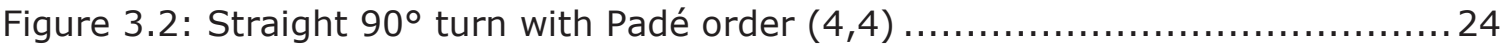

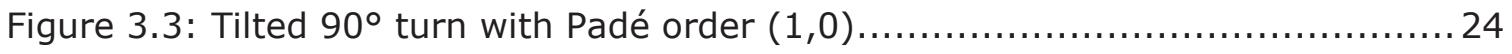

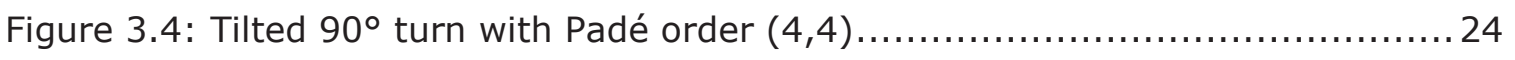

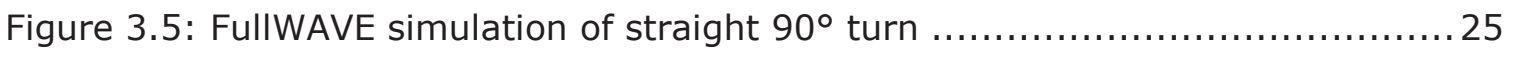

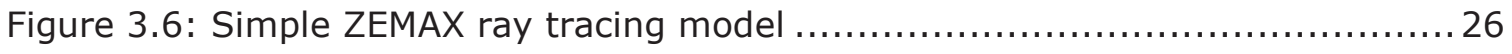

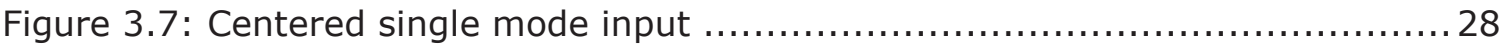




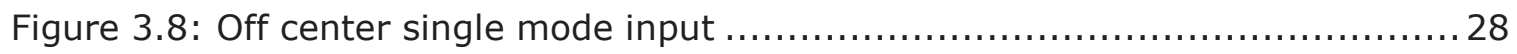

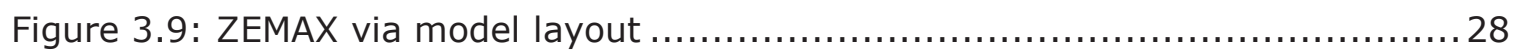

Figure 5.1: Selection of modes in $15 \mathrm{~mm}$ straight with centered single mode input 36

Figure 5.2: $15 \mathrm{~mm}$ straight with centered single mode input mode profile and cross

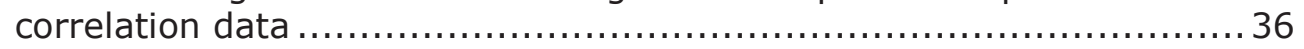

Figure 5.3: Power output after via propagation for $250 \mu \mathrm{m}$ lead-in (center SM input) 38

Figure 5.4: Power output after via propagation for $500 \mu \mathrm{m}$ lead-in (center SM input)

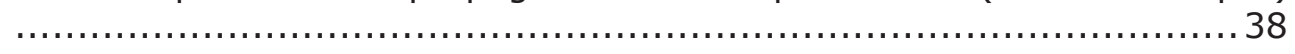

Figure 5.5: Selection of modes in $15 \mathrm{~mm}$ straight with off center single mode input39

Figure 5.6: $15 \mathrm{~mm}$ straight with off center single mode input mode profile and cross

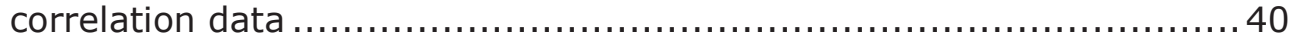

Figure 5.7: $15 \mathrm{~mm}$ straight with off center single mode input modal distributions

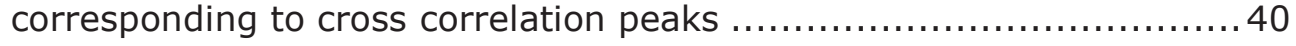

Figure 5.8: Power output after via propagation for $250 \mu \mathrm{m}$ lead-in (off center SM

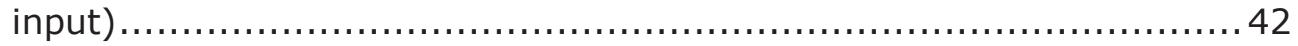

Figure 5.9: Power output after via propagation for $500 \mu \mathrm{m}$ lead-in (off center SM

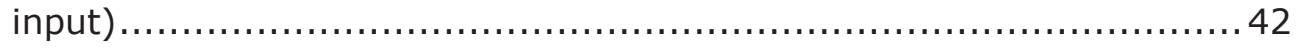

Figure 5.10: Selection of modes for $15 \mathrm{~mm}$ straight with $50 \mu \mathrm{m}$ multimode input... 43

Figure 5.11: $15 \mathrm{~mm}$ straight with multimode input mode profile and cross correlation

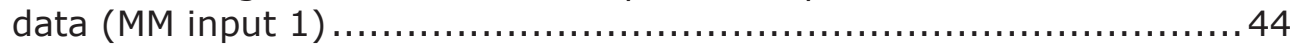

Figure 5.12: $15 \mathrm{~mm}$ straight with multimode input mode profile and cross correlation

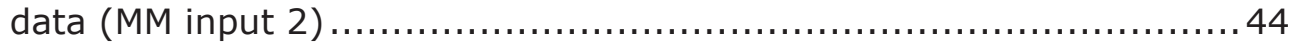

Figure 5.13: Power output after via propagation for $250 \mu \mathrm{m}$ lead-in (MM input 1) ...45

Figure 5.14: Power output after via propagation for $500 \mu \mathrm{m}$ lead-in (MM input 1) .. 45

Figure 5.15: Power output after via propagation for $250 \mu \mathrm{m}$ lead-in (MM input 2) .. 46

Figure 5.16: Power output after via propagation for $500 \mu \mathrm{m}$ lead-in (MM input 2) .. 46

Figure 5.17: Power output after via propagation for $0 \mu \mathrm{m}$ lead-in (MM input 2) .....4 47 


\section{List of Tables}

Table 3.1: Padé approximant simulation parameters................................ 23

Table 3.2: RSoft mode generating simulation parameters.......................... 27

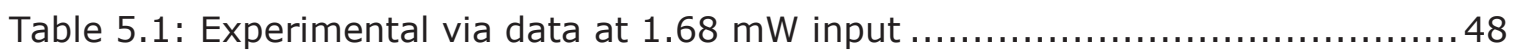





\section{List of Equations}

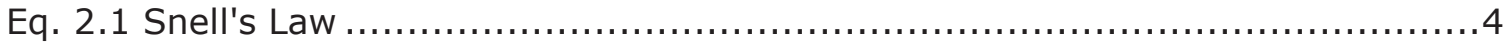

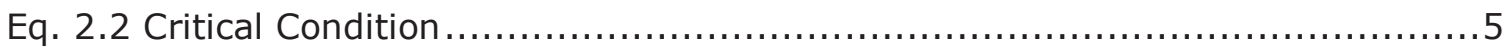

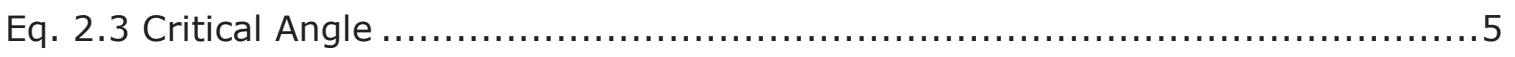

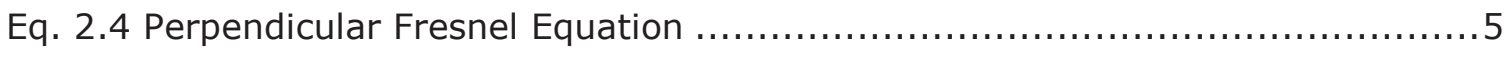

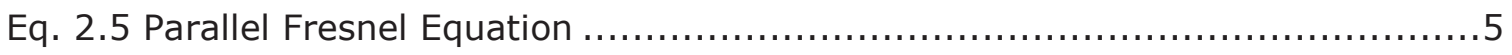

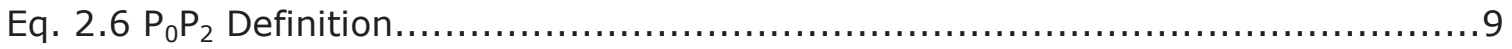

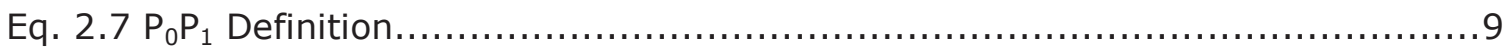

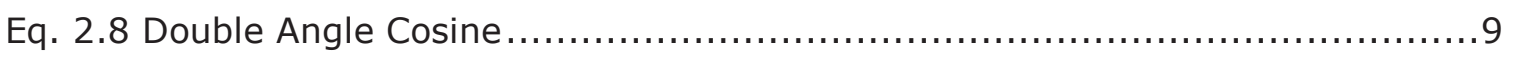

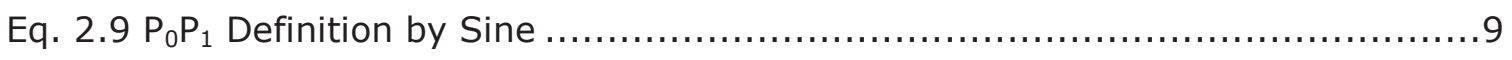

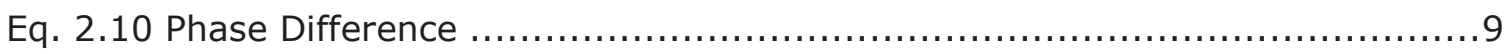

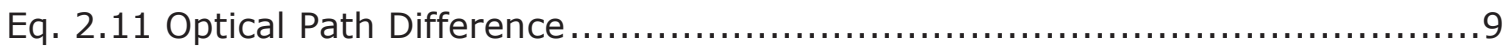

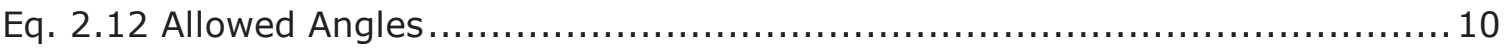

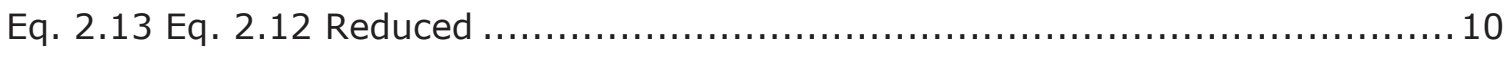

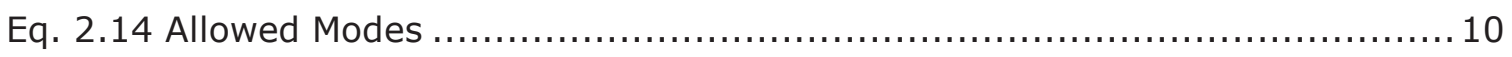

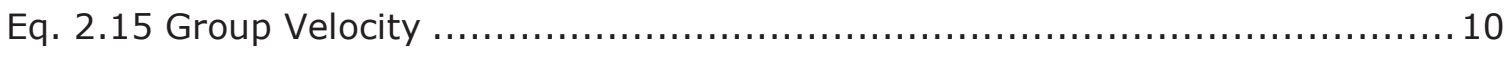

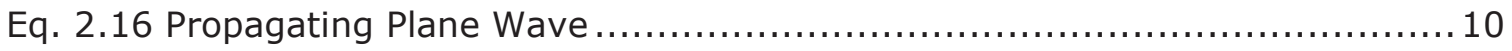

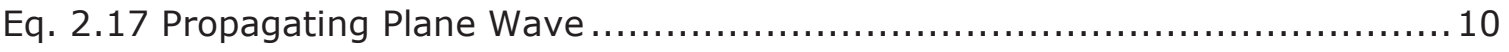

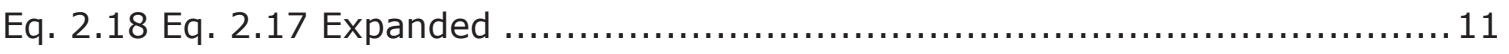

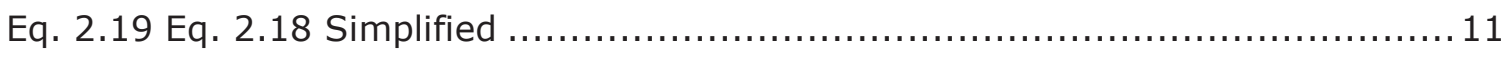

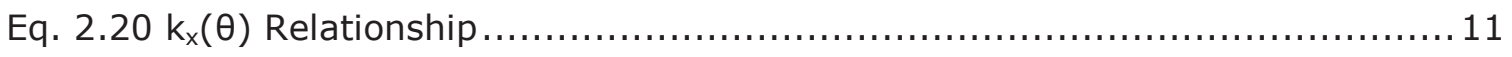

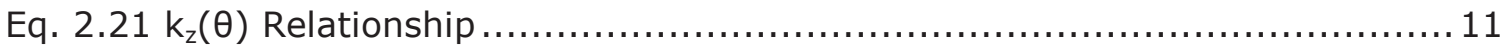

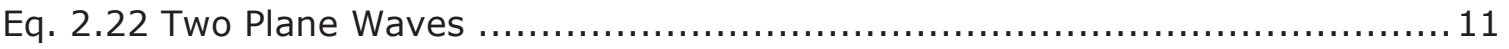




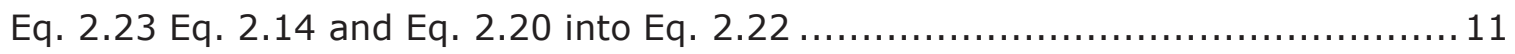

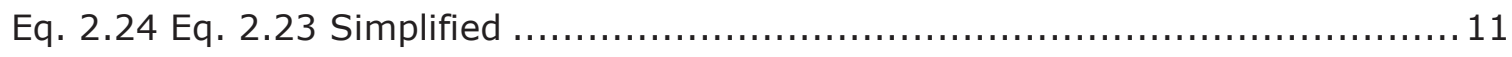

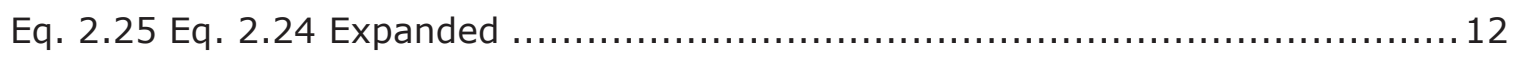

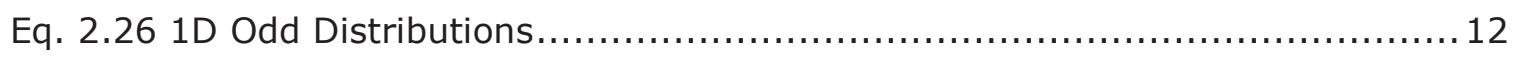

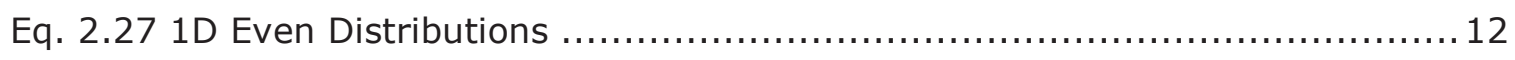

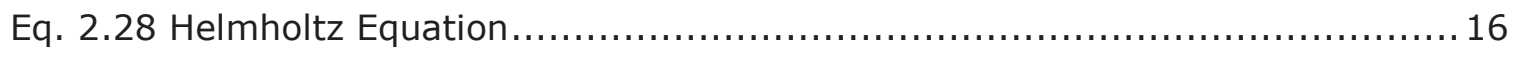

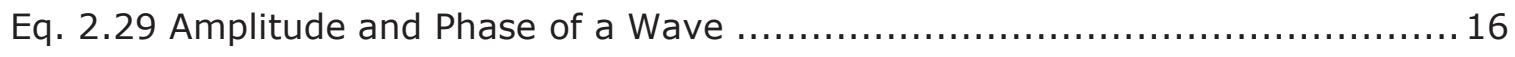

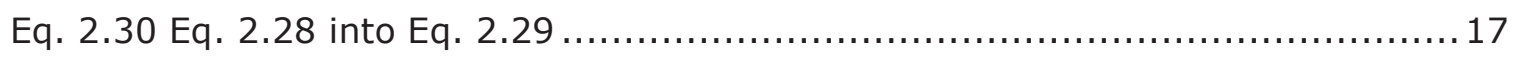

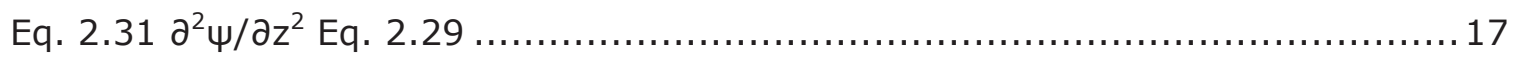

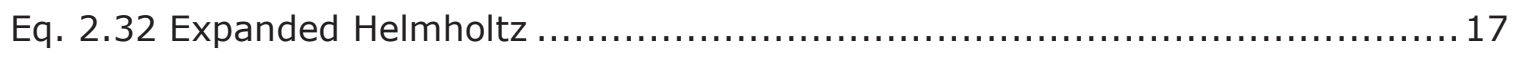

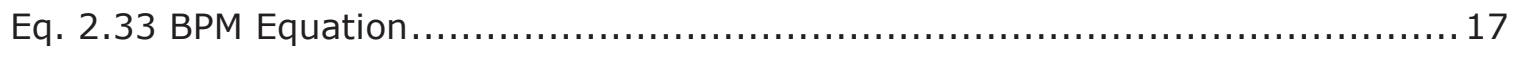

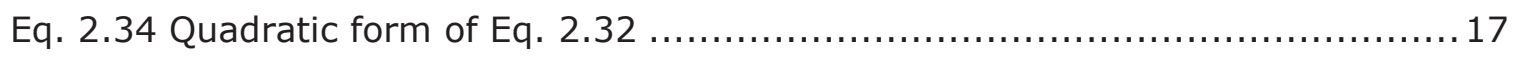

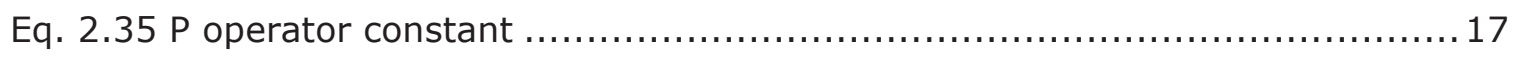

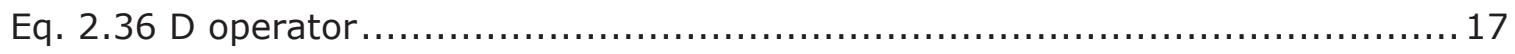

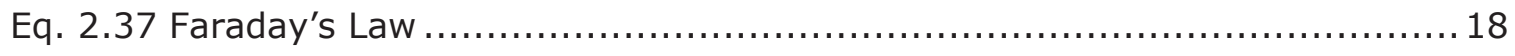

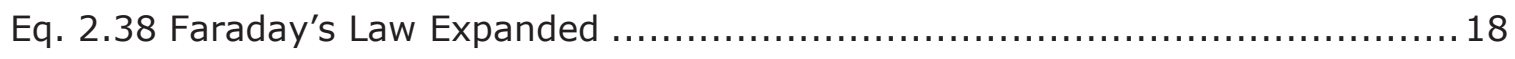

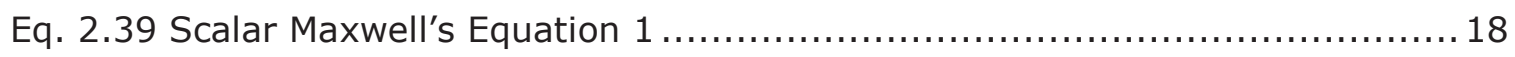

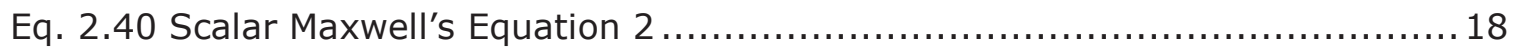

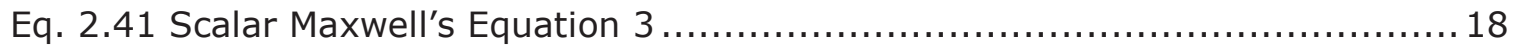

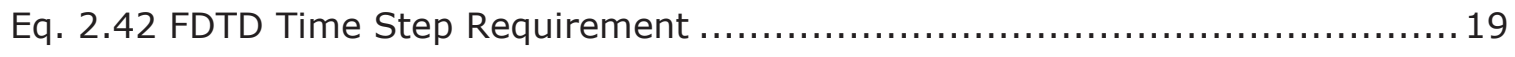

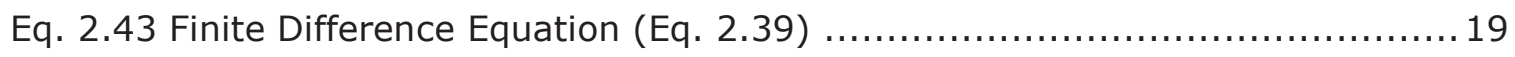

Eq. 2.44 Fresnel Number......................................................... 20 


\section{Acknowledgements}

I am incredibly indebted to my advisor Dr. Christopher Middlebrook, who provided his support through my undergraduate degree and encouraged me to continue in pursuit of this graduate degree. He pushed me when I needed pushing, taught me when I needed teaching, and was always available when I needed his support in any way. His investment in me and other students is invaluable.

I am also grateful to my committee, Dr. Michael Roggemann, Dr. Craig Friedrich, and Dr. Warren Perger for their support and advice.

I would also like to thank Dr. Anand Kulkarni and Dr. George Semouchkin for their inspiring and infectious passion for knowledge demonstrated through their graduate course instruction.

I thank Dr. Karl Walczak and my fellow graduate students, Kevin Kruse, Nick Riegel, Brandon Swatowski, and Weston Thomas for listening to my thoughts and providing valuable insights into countless ideas and topics.

I wish to thank the many friends that have been very supportive in keeping me sane and on the right track; especially Scott Austin, Pete Elenbaas, Andrew Korcal, Evan Johnson, Andy Nauta, and Jon Salinas as well as meinen Brüdern Thorsten und JensPeter Nasse.

My brothers Mitchell and Grant have always been there for me as comic relief, punching bag stand-ins, fellow adventurers, and true brothers.

My parents Scott and Pat have supported me in every endeavor for my entire life. Their constant love has helped me get this far. Their excitement for my success and enthusiasm for my future have never ceased to urge me onward. Their advice has proven itself priceless on many occasions.

Lastly, but most importantly, I joyfully give thanks to God who strengthens me with great endurance and patience and apart from whom I can do nothing. 



\section{Abstract}

Embedded siloxane polymer waveguides have shown promising results for use in optical backplanes. They exhibit high temperature stability, low optical absorption, and require common processing techniques. A challenging aspect of this technology is out-of-plane coupling of the waveguides. A multi-software approach to modeling an optical vertical interconnect (via) is proposed. This approach utilizes the beam propagation method to generate varied modal field distribution structures which are then propagated through a via model using the angular spectrum propagation technique. Simulation results show average losses between 2.5 and $4.5 \mathrm{~dB}$ for different initial input conditions. Certain configurations show losses of less than $3 \mathrm{~dB}$ and it is shown that in an input/output pair of vias, average losses per via may be lower than the targeted $3 \mathrm{~dB}$. 



\section{Chapter 1}

\section{Introduction}

Embedded polymer multimode optical waveguides show promising results for use in optical backplane. Siloxane polymers are a common choice for fabricating embedded polymer waveguides[1]-[3]. These materials are ultraviolet (UV) cured, which makes them easy to fabricate using photolithography or other UV curing techniques. They have low optical absorption and also demonstrate high thermal stability, which allows them to go through the high temperature manufacturing steps required for electrical circuit boards and to operate in high temperature environments.

The most challenging aspect of this technology before wide implementation is high performance of out-of-plane coupling of the waveguides. The coupling efficiency will be dependent upon the components used, for example, vertical cavity surface emitting laser (VCSEL) transceivers and MT-style fiber connectors.

A fabrication method has been proposed for creating an optical vertical interconnect assembly (via), which would allow surface components to gain access to the waveguides embedded in the optical printed wiring board (OPWB) using a system of 45 degree micro-mirrors and ball lenses. The efforts of this research are focused on simulation and modeling of the proposed via in order to characterize the losses and guide in the overall design for maximum coupling efficiency.

The beam propagation method implemented in RSoft is used to create a variety of modal structures which are used as inputs to a ZEMAX simulation to calculate the field after propagation through the via structure. MATLAB code is then used to calculate the total power in a desired area, which can then be used to evaluate the losses incurred through the via structure. This provides a collection of values corresponding to losses depending on the modal structure of the field inside the waveguide before the via.

By averaging the losses for the different mode structures, the expected transmitted power can be determined for a random input. This indicates the overall performance of the via and can be used in determining a final link budget for an entire system. This research indicates that an average of $3 \mathrm{~dB}$ loss per via for an input/output via pair is possible with the current via design. Performance with a single mode source input indicate the potential for very low losses and output from a multimode waveguide show less than $5 \mathrm{~dB}$ losses. This pair can then have losses of at or below $3 \mathrm{~dB}$. 



\section{Chapter 2}

\section{Background}

\subsection{Optical Communication Systems}

Optical communications offer a range of benefits including larger bandwidth, energy concentration and subsequent increase of signal to noise ratio [4], and immunity to electromagnetic interference. The higher bandwidth is a result of the increased carrier frequency. Radio frequencies cover the spectrum into the gigahertz range while optical frequencies are in the terahertz range. Radio Frequency (RF) domain communication systems can operate at carrier frequencies up to hundreds of gigahertz. Wired communication systems that employ various transmission lines are limited at these higher frequencies by the skin effect on resistance, which causes a rise in resistance with a rise in frequency. When the frequency reaches $10 \mathrm{GHz}$, the transmission losses become significant in terms of signal to noise ratio [5]. In the age of low power devices and communication link budgets, it is not applicable to simply increase transmitter power to receive the signal through the resistive losses. In order to achieve higher bandwidth data transmission rates, an alternative method to avoid the losses should be used and implementation of optical communications should be exploited.

RF communication systems suffer from the fact that their wired transmission lines also act as antennas. This provides the opportunity for interference from other RF sources. This is not present in guided optical wave communication systems because the wave is contained within the channel and is unaffected by other electromagnetic radiation sources, both in the RF domain and the optical domain. Crossing of waveguides can be done on the same plane with negligible crosstalk between the channels. This provides additional communication security as well as new board design possibilities.

Optical waveguides allow a large amount of energy (signal) to be confined within a small area. Single mode fibers are on the order of microns and multimode fibers are only tens of microns in size. Optical fibers have long been the preferred method for long distance communication due to their ability to transmit light at a loss of only around $3.5 \%$ per kilometer [6]. This energy concentration allows both higher signal to noise ratios and smaller component size.

Multimode fibers have also been put into use in short distance communications. They are larger than single mode fibers and easier to make, however they do not perform when used as long distance channels due to pulse broadening. Pulse 
broadening is due to the different group velocities of the modes present in the waveguide. This is described in more detail in section 2.2.2.2. However, when only short distances are required, multimode fibers are a cheaper option while retaining the significant benefits of a fiber system. As the demand for data and bandwidth rises, electrical communications systems need to be replaced with higher bandwidth systems. Over the relatively short distances required in computer backplanes, multimode waveguides offer an increased bandwidth and lack of electromagnetic interference - both important steps in improving a communications system.

\subsection{Waveguide Theory}

Four theories of optics have evolved to explain the complex phenomena therein. These are geometric, wave, electromagnetic, and quantum optics, in order from simplest to most complex. Each higher level theory explains all phenomena explained by the lower level theories and provides explanations for things that can't be explained with the lower level theories. This thesis will present waveguide theory using ray (geometric) and wave optics. Each step should provide the reader with a more complete understanding of the topic and facilitate understanding of the motivation and methods behind this work.

Electromagnetic theory is not described in detail here, because it is not required to understand the basic principles behind waveguides and mode structures. It is used briefly in section 2.2.1.1 as a secondary explanation of total internal reflection and also in the mathematical methods in Chapter 3.

\subsubsection{Geometric Optics}

Geometric optics is an approximation that is valid when the wavelength in use is very small. It can be validated by using a wave optics approach and allowing the wavelength to become infinitesimally small [6]. For this reason, it is a good tool to gain a basic understanding of some of the concepts presented in this thesis.

\subsubsection{Total I nternal Reflection}

One of the fundamental laws of geometric optics is the Law of Refraction. It states that light incident on a dielectric interface of two media of different refractive indices will bend at that interface. This law is governed by Snell's Law (Eq. 2.1), which is a mathematical description of the refraction geometry at an interface, illustrated in Error! Reference source not found..

$$
n_{1} \sin \theta_{1}=n_{2} \sin \theta_{2} \quad \begin{array}{r}
\text { Snell's } \\
\text { Law }
\end{array}
$$




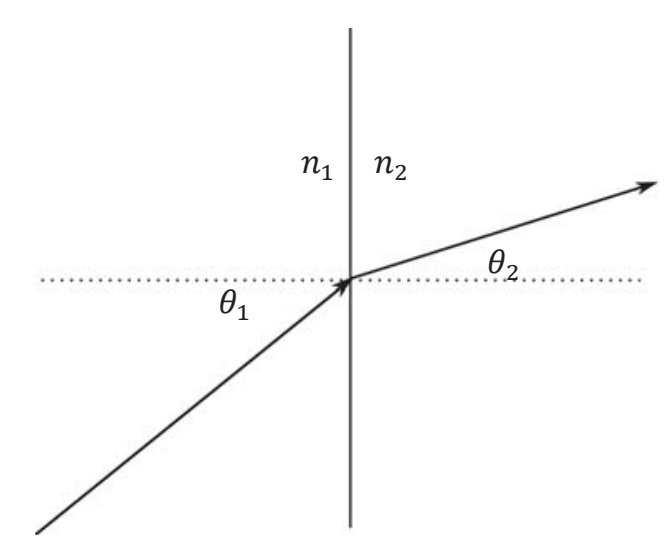

\section{Figure 2.1: Snell's Law}

When $n_{1}>n_{2}$ and $\theta_{1}$ is in a specific range, it is possible to achieve total reflection (instead of refraction) at such an interface. This range is defined by the lowest angle at which all light is reflected, known as the critical angle. The critical angle is mathematically defined as the smallest angle $\theta_{1}$ where $\theta_{2}$ begins to be undefined in Snell's law.

$$
\begin{aligned}
\sin \theta_{2} & >1=\frac{n_{1} \sin \theta_{c}}{n_{2}} \\
\theta_{c} & =\sin ^{-1} \frac{n_{2}}{n_{1}}
\end{aligned}
$$
Critical Condition

Eq. 2.3 Critical Angle

Total internal reflection can also be described in the electromagnetic approach using the Fresnel equations in Eq. 2.4 and Eq. 2.5.

$$
\begin{gathered}
r_{\perp}=\frac{n_{i} \cos \left(\theta_{i}\right)-n_{t} \cos \left(\theta_{t}\right)}{n_{i} \cos \left(\theta_{i}\right)+n_{t} \cos \left(\theta_{t}\right)} \\
r_{\|}=\frac{n_{t} \cos \left(\theta_{i}\right)-n_{i} \cos \left(\theta_{t}\right)}{n_{i} \cos \left(\theta_{t}\right)+n_{t} \cos \left(\theta_{i}\right)}
\end{gathered}
$$

At incident angles higher than the critical angle, the phenomenon known as total internal reflection occurs. At this point, $100 \%$ of the light is reflected back inside the material. The Fresnel equations along with Snell's Law are graphed in Figure 2.2 for a water $(n=1.33)$ and air $(n=1)$ interface. The critical angle $\theta_{c}$ is also shown. 


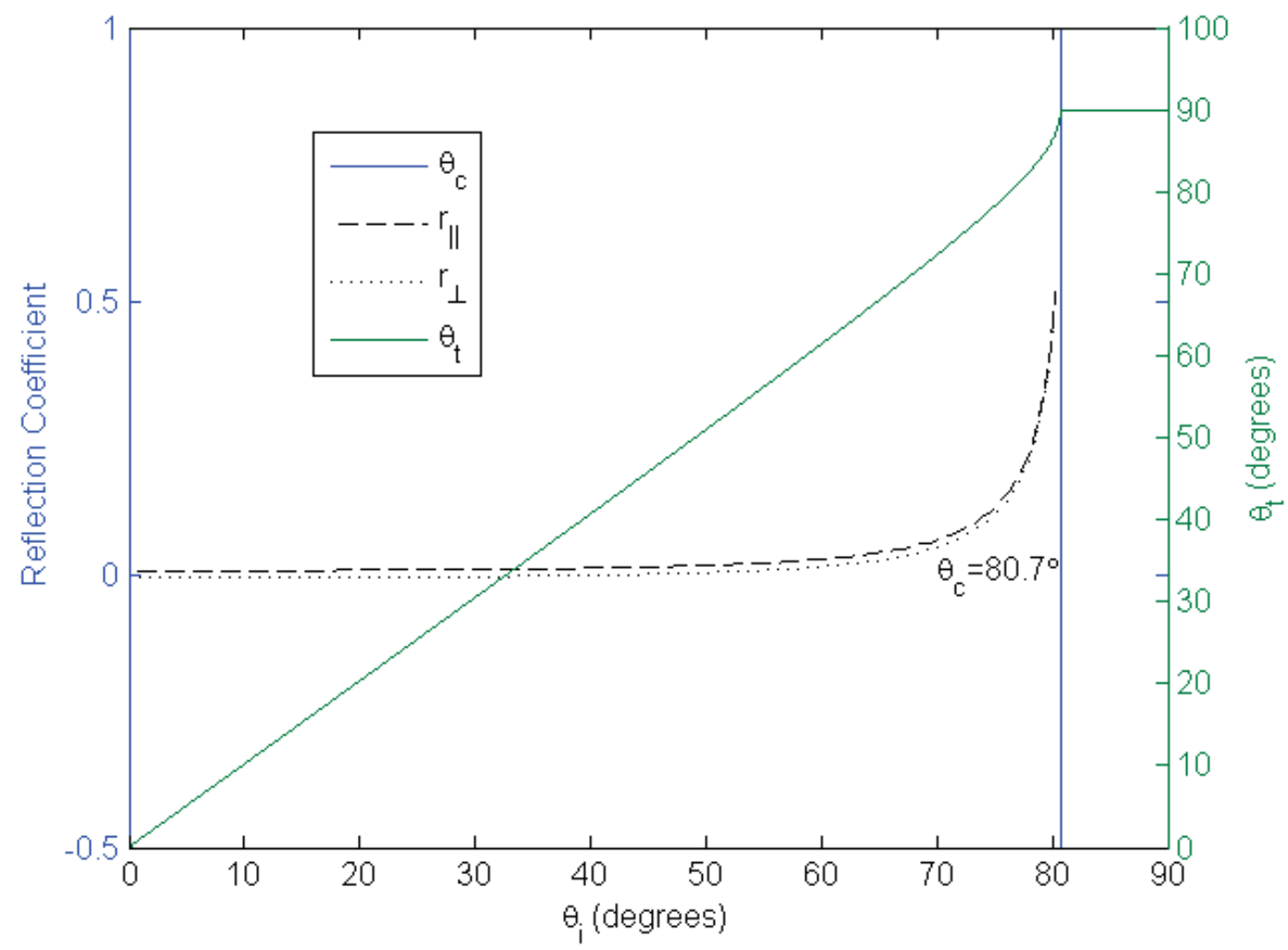

Figure 2.2: Critical angle by Snell's Law and Fresnel Coefficients

\subsubsection{Total I nternal Reflection Waveguides}

It is possible to take advantage of the total internal reflection phenomenon by creating a structure in which propagating light will be trapped within a material. This can be simply demonstrated by the material profile depicted in Figure 2.3. It can be seen that some rays will escape the dielectric slab, but some will reflect at the dielectric interface at both the top and the bottom of the slab. These rays will stay in the material for its entire length or until the slab thickness changes or a different interface is encountered.

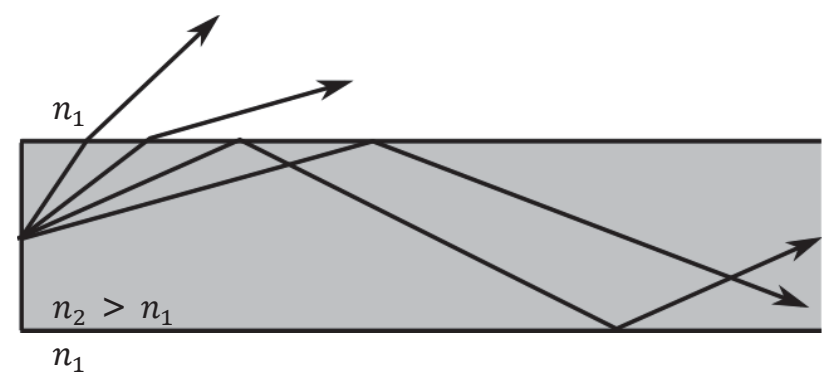

Figure 2.3: Total internal reflection in a dielectric slab 
As was seen in Figure 2.2, light incident on a total internal reflection capable interface which is incident at an angle lower than the critical angle will still have a partial reflection. While these rays do stay in the guide for some bounces, the successive bounces and partial reflections will attenuate the ray and it is not said to be guided.

This simple two-dimensional concept easily translates into three dimensions. As long as the propagating rays strike the interfaces at or above the critical angle, total internal reflection can occur and the light will propagate through the material. This can be true only when the inner material, known as the core, has a higher refractive index than the outer material, known as the cladding. An example of a three dimensional structure of this type is the rectangular waveguide cross section pictured in Figure 2.4. This type of structure confines light in both axes of the plane perpendicular to the direction of propagation.

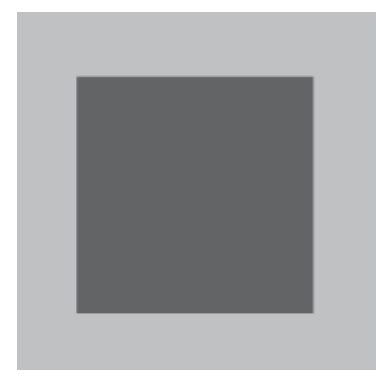

Figure 2.4: Rectangular waveguide profile

\subsubsection{Wave Optics}

The theory of wave optics describes light as a propagating wave with some finite wavelength; however it does not take into account polarization vectors that are used in the electromagnetic approach to optics. Instead of seeing light propagating as a ray, a wave propagates along the path of a ray as depicted in Figure 2.5. The wave is defined by its distance between two repeated points, such as from peak to peak or trough to trough. Planes perpendicular to the direction of propagation at these repeating points can be thought of as wavefronts. These wavefronts simply represent a repeating location of equal phase along the wave, also pictured in Figure 2.5 as a dotted line. 


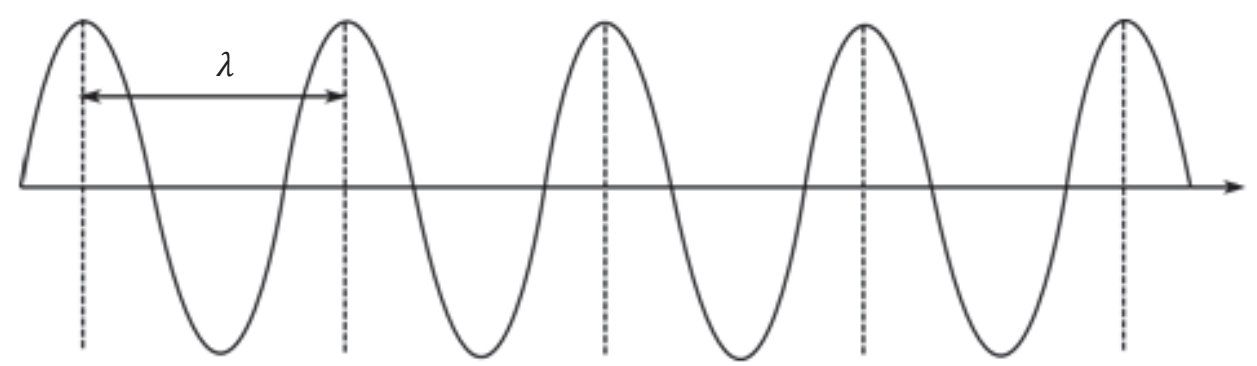

Figure 2.5: Wavefronts of an oscillating wave

It is then possible to modify the ray diagram of a total internal reflection waveguide in Figure 2.3 to show the wavefronts. This can be seen in Figure 2.6.

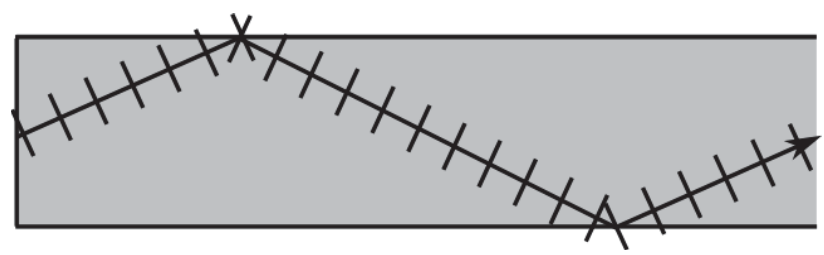

Figure 2.6: Wavefronts propagating in a waveguide

\subsubsection{Self-consistency Condition and Modes}

In order to transfer information from one end of a waveguide to the opposite end, the wave needs to constructively interfere with itself, which requires they meet a self consistency condition and repeat themselves [7]. If the wave repeats itself after every second bounce, it will do so for the length of the guide. Depending on the geometry of the waveguide, wavefronts propagating at different angles will be unable to reproduce themselves as in Figure 2.7 or be able to reproduce the wavefronts as seen in Figure 2.8.
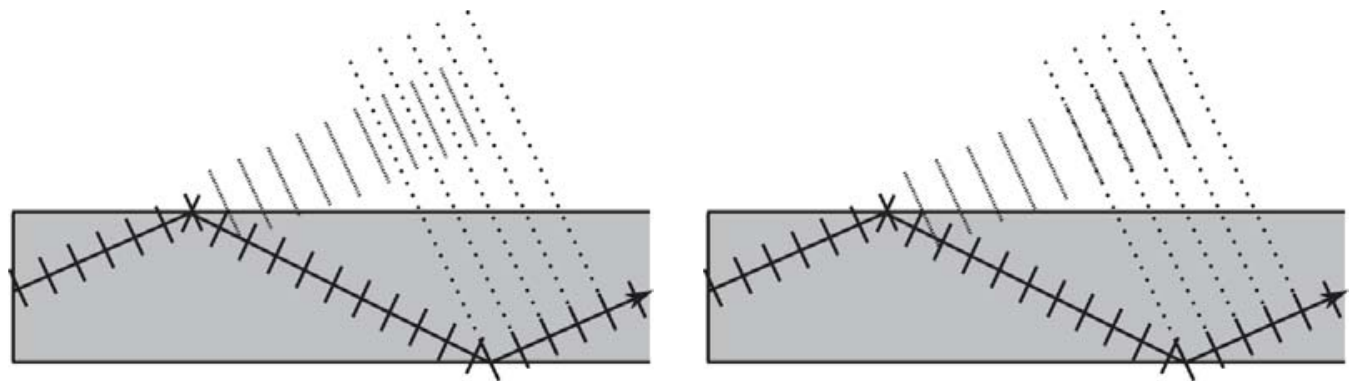

Figure 2.7: Non-repeating wavefronts Figure 2.8: Repeating wavefronts

To determine what propagation angles cause the wave to reproduce itself, the geometry in Figure 2.9 must be considered. It should be noted that this $\theta$ is not defined in the same way as was used for Snell's Law. In this case, it is in reference 
to the wave and the surface, while in Snell's Law it is defined as the angle between the wave and the surface normal.

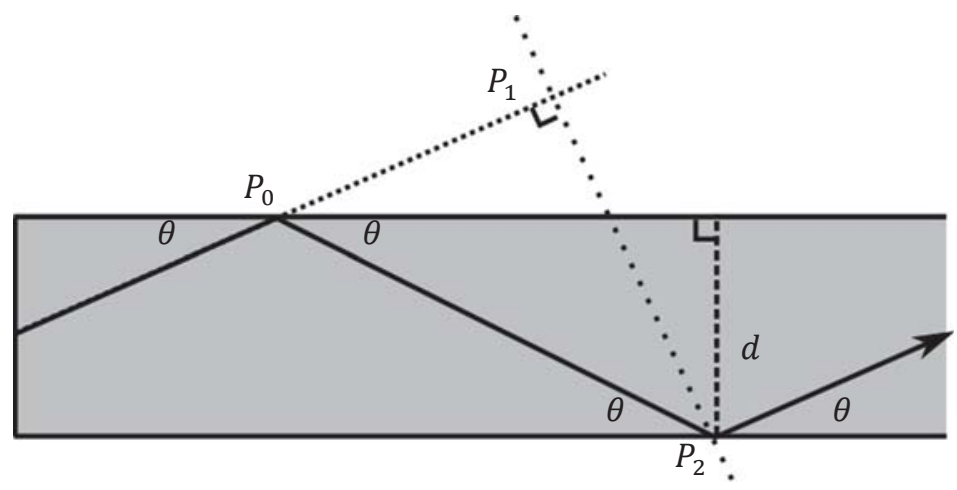

Figure 2.9: Self-consistency geometry
$P_{0}$ - Point of reflection 1

$P_{1}$ - Point of wavefront overlap

$P_{2}$ - Point of reflection 2

$\theta$ - Angle of incidence

$d$ - Guide size

$\lambda$ - Wavelength

The following trigonometric relations can be gathered from this diagram.

$$
\begin{gathered}
\sin \theta=\frac{d}{\overline{P_{0} P_{2}}} \therefore \overline{P_{0} P_{2}}=\frac{d}{\sin \theta} \\
\cos 2 \theta=\frac{\overline{P_{0} P_{1}}}{\overline{P_{0} P_{2}}} \therefore \overline{P_{0} P_{1}}=\overline{P_{0} P_{2}} \cos 2 \theta
\end{gathered}
$$

$P_{0} P_{1}$ Definition

Using the double angle cosine formula, the following expression for $\overline{P_{0} P_{1}}$ can be found.

$$
\begin{gathered}
\cos 2 \theta=1-2 \sin ^{2} \theta \\
\overline{P_{0} P_{1}}=\overline{P_{0} P_{2}}-2 \overline{P_{0} P_{2}} \sin ^{2} \theta
\end{gathered}
$$
Double Angle Cosine

Eq. 2.9 $\mathrm{P}_{0} \mathrm{P}_{1}$ Definition by Sine

During the propagation from $P_{0}$ to $P_{2}$, the wave must develop a phase difference $(\Delta \phi)$ with the propagation which would occur from $P_{0}$ to $P_{1}$ of $2 \pi n$, where $n=0,1,2, \ldots$. Additionally, each reflection will induce a $\pi$ phase shift, resulting in a total phase shift of $2 \pi$ due to the two reflections.

$$
\begin{gathered}
\Delta \phi=\frac{2 \pi}{\lambda} \overline{P_{0} P_{2}}-\frac{2 \pi}{\lambda} \overline{P_{0} P_{1}}-2 \pi=2 \pi n \\
\overline{P_{0} P_{2}}-\overline{P_{0} P_{1}}=(n+1) \lambda
\end{gathered}
$$

Phase Difference

Eq. 2.11 Optical Path Difference 
By combining Eq. 2.6, Eq. 2.9, and Eq. 2.11, the following relation and simplification can be made.

$$
\begin{array}{rr}
\frac{d}{\sin \theta}-\left(\frac{d}{\sin \theta}-2 \frac{d}{\sin \theta} \sin ^{2} \theta\right)=(n+1) \lambda & \begin{array}{r}
\text { Eq. } 2.12 \\
\text { Allowed Angles }
\end{array} \\
2 d \sin \theta=(n+1) \lambda=m \lambda & \text { Eq. } 2.13 \\
m=1,2,3, \ldots & \text { Eq. } 2.12 \text { Reduced }
\end{array}
$$

This equation indicates that there are discrete angles $\theta$ corresponding to each integer value of $m$ that are allowed to propagate as long as $\theta_{m}<\frac{\pi}{2}-\theta_{c}$. These are known as modes and can be described by Eq. 2.14.

\subsubsection{Group Velocity}

$$
\sin \theta_{m}=\frac{m \lambda}{2 d}
$$

Eq. 2.14

Allowed Modes

All light in the medium travels at the same velocity, $v=\frac{c}{n}$, but since each mode is at a different angle, it will have different absolute propagation length to travel the same distance down the waveguide as other modes. The velocity at which the mode propagates with respect to the waveguide is known as the group velocity. The group velocity is a function of the velocity of light in the medium and the mode angle, given in Eq. 2.15.

$$
v_{m}=v \cos \left(\theta_{m}\right)
$$

Eq. 2.15

Group Velocity

Since each mode is travelling at a different velocity with respect to the waveguide itself, a pulse of light composed of different modes will spread out over the propagation distance. If two pulses are sent too close together, they will begin to overlap and no longer be distinguishable. This is the reason multimode waveguides are not used for long distance communications.

\subsubsection{Distribution of Fields}

The amplitude of a propagating plane wave as described in section 2.2.2.1 may be mathematically described by the following equations [8]. The coordinates are defined that the $x-y$ plane corresponds to a cross-section of the waveguide with origin at the center of the structure and the length of the waveguide is along the zaxis.

$$
\begin{array}{lr}
E=E_{0} \exp (j(\vec{k} \cdot \vec{r}-\omega t)) & \begin{array}{r}
\text { Eq. } 2.16 \\
\text { Propagating Plane Wave }
\end{array} \\
E=E_{0} \exp (j \omega t) \exp (j \vec{k} \cdot \vec{r}) & \text { Eq. } 2.17
\end{array}
$$




$$
E=E_{0} \exp (j \omega t) \exp \left(j\left(k_{x} x+k_{y} y+k_{z} z\right)\right)
$$

Eq. 2.18

Eq. 2.17 Expanded

We are interested in looking at the field distribution inside a waveguide at a given time and position $(t, z)$. For simplicity, we will take $E_{0}=1, t=0$, and $z=0$. This simplification is shown in Eq. 2.19.

$$
E=\exp \left(j\left(k_{x} x+k_{y} y\right)\right)
$$

Eq. 2.18

Simplified

We will first look at the two-dimensional case where $k_{y}=0$. We can then define $k_{x}$ and $k_{z}$ in terms of $\theta$ using Figure 2.10, Eq. 2.20 and Eq. 2.21.

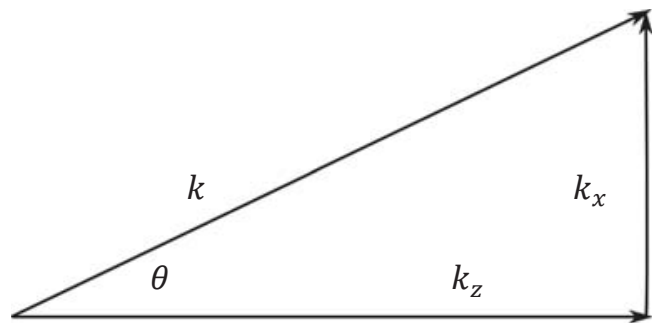

Figure 2.10: Propagation vector relationship to $\theta$

$$
\begin{aligned}
& k_{x}=k \sin \theta=\frac{2 \pi}{\lambda} \sin \theta \\
& k_{z}=k \cos \theta=\frac{2 \pi}{\lambda} \cos \theta
\end{aligned}
$$

Relationship

Eq. 2.21

Relationship

It is important to keep in mind that there are two waves propagating at once for any given $\theta$ with $\pm k_{x}$ and valid $\theta$ values are the previously defined $\theta_{m}$ values. For this reasoning, the following simplifications can be made to the field distribution $E$ in combination with Eq. 2.14.

$$
\begin{gathered}
E=\exp \left(j k_{x} x\right)+\exp \left(-j k_{x} x\right) \\
E=\frac{1}{2} \exp \left(j x \frac{2 \pi}{\lambda} \frac{m \lambda}{2 d}\right)+\frac{1}{2} \exp \left(-j x \frac{2 \pi}{\lambda} \frac{m \lambda}{2 d}\right) \\
E=\frac{1}{2} \exp \left(j x \frac{m \pi}{d}\right)+\frac{1}{2} \exp \left(-j x \frac{m \pi}{d}\right)
\end{gathered}
$$

Eq. 2.22

Two Plane Waves

Eq. 2.23

Eq. 2.14 and Eq. 2.20 into Eq. 2.22

Eq. 2.24

Eq. 2.23 Simplified 


$$
\begin{gathered}
E=\frac{1}{2} \cos \left(x \frac{m \pi}{d}\right)+j \frac{1}{2} \sin \left(x \frac{m \pi}{d}\right) \\
+\frac{1}{2} \cos \left(-x \frac{m \pi}{d}\right)+j \frac{1}{2} \sin \left(-x \frac{m \pi}{d}\right) \\
E=\cos \left(x \frac{m \pi}{d}\right), m=1,3,5, \ldots \\
E=\sin \left(x \frac{m \pi}{d}\right), m=2,4,6, \ldots
\end{gathered}
$$

Eq. 2.24 Expanded

Eq. 2.26

1D Odd

Distributions

Eq. 2.27

1D Even

Distributions

Eq. 2.26 and Eq. 2.27 are graphed in Figure 2.11 for $d=20 \mu \mathrm{m}$ and $\lambda=850 \mathrm{~nm}$.
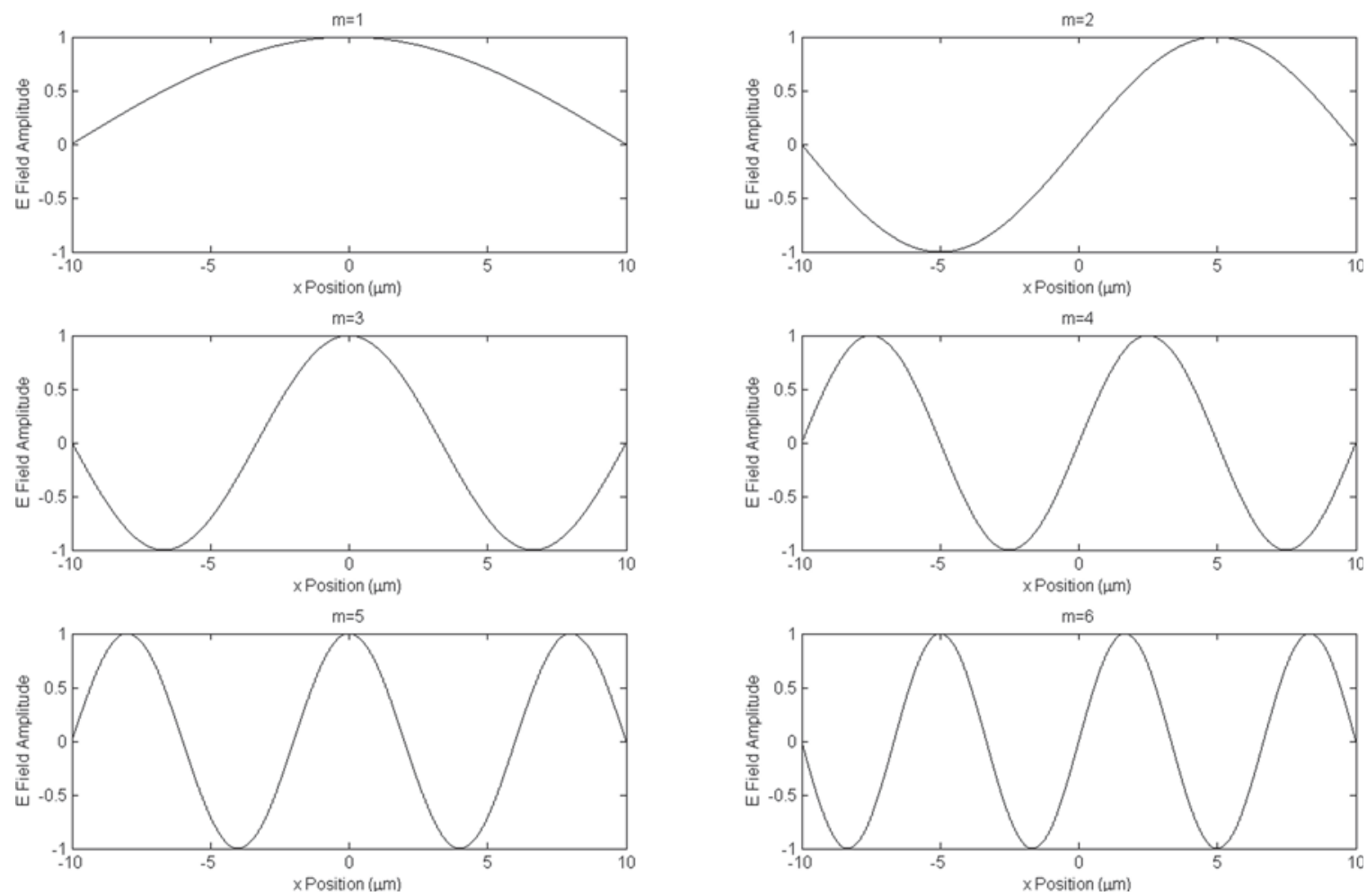

Figure 2.11: One dimensional mode distributions

Two dimensional distributions for a square waveguide can be found by leaving $k_{y}$ in Eq. 2.22 and following the same process. Essentially what happens is the one dimensional mode defined in Eq. 2.26 or Eq. 2.27 for $x$ is multiplied by the one dimensional mode for $y$. It is possible to have the mode numbers for $x$ and $y\left(m_{x}\right.$ and $m_{y}$ ) be different. A selection of these two dimensional equivalent distributions is shown in Figure 2.12. 

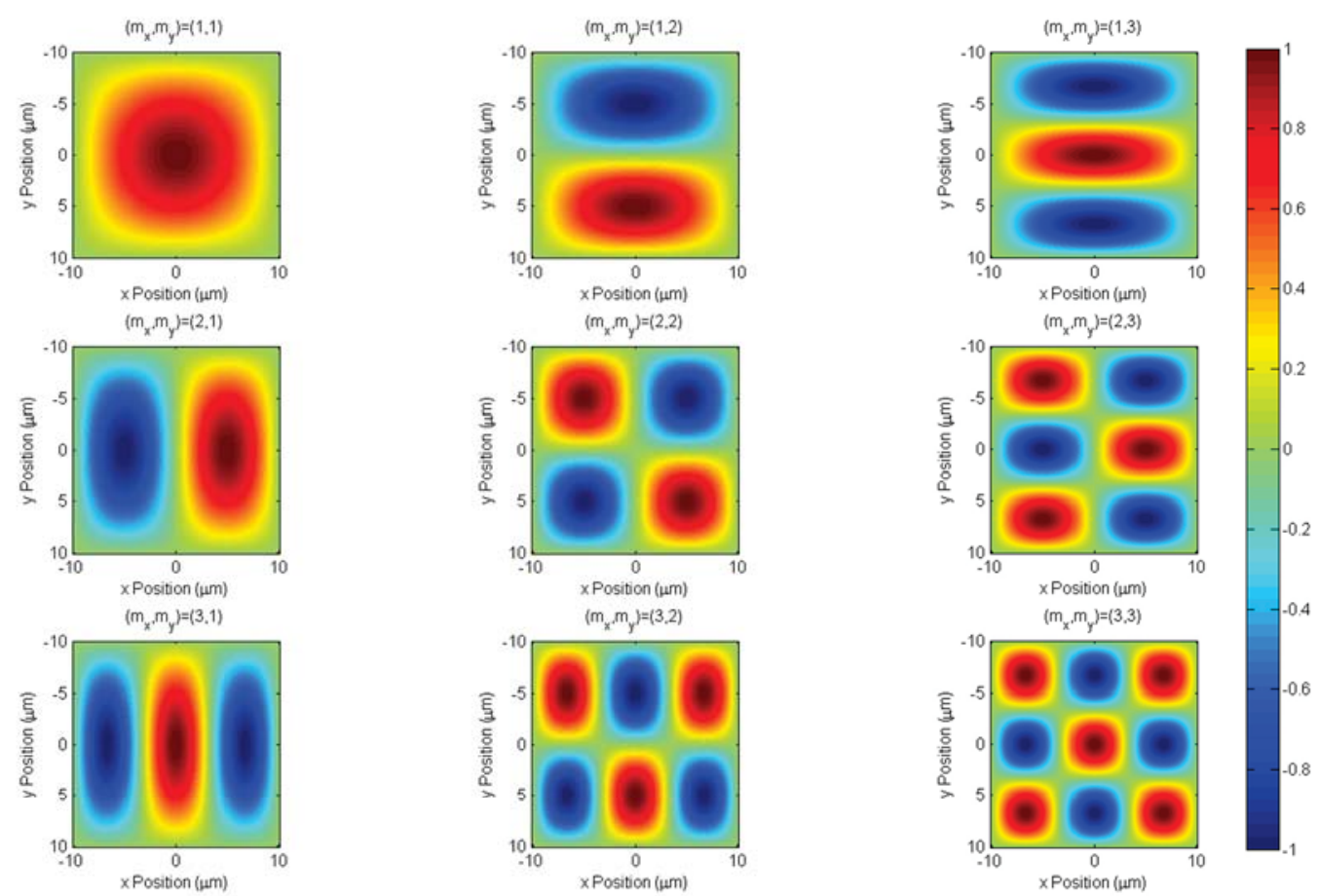

Figure 2.12: Normalized two dimensional mode field distributions

\subsection{Project Parameters}

In order for these simulations to be accurate, modeling parameters were chosen to be consistent with the work being done in fabrication. These parameters include choice of material, waveguide size, and optical testing conditions. These conditions were pre-determined and not evaluated in this research. The merits of each determination will be briefly touched on in these sections, but not discussed in full detail.

\subsubsection{Siloxane Polymers}

Siloxane polymers (also known as polysiloxanes or silicones) are formed when silicon and oxygen atoms chain together with hydrogen atoms or hydrocarbon groups (alkanes) bonded to the silicon atoms. This change in structure is responsible for the thermal stability of these types of materials are known for, withstanding degradation to above $350^{\circ} \mathrm{C}$ in some cases. By selecting certain types of hydrocarbon groups attached to the silicon, the thermal properties can be further improved as these groups may increase the strength of the Si-O bonds [9].

The materials in use for fabrication on this project are siloxane polymers OE-4140 (core) and OE-4141 (clad), produced by Dow Corning. The solutions used in fabrication are composed of the siloxane monomer and toluene solvent. The 
monomers are polymerized by UV exposure and the toluene is removed through preand post-bake procedures.

These materials were chosen for their low optical loss $(0.03-0.05 \mathrm{~dB} / \mathrm{cm}$ at $850 \mathrm{~nm})$, high temperature tolerance, and preferred processing methods. When cured, the core material has a refractive index of 1.53 and the cladding material has a refractive index of 1.51 [10].

\subsubsection{Waveguide Definition}

A layer of cladding is initially spun on the substrate and cured. This is done to provide a bottom cladding layer as well as to create a planarized surface for the waveguides. On top of this cladding layer, a thin layer of core material is spun on. This material is then selectively cured using ultraviolet definition techniques. Photolithography and a laser direct write system have both been employed to define these waveguides. Each waveguide is defined to be $50 \mu \mathrm{m}$ by $50 \mu \mathrm{m}$. Different structures have been fabricated, including straights, 90 degree turns, spirals, and various others. It has been the goal to fabricate these structures and characterize the losses associated with each of them. After the core has been defined and cured, a top cladding layer is spun on and cured. The entire system is then baked again to remove the remaining solvent [11].

Using a direct dispense method, less material can be used in manufacturing a board. Material is only placed on the substrate using a small syringe where it will be used to define a waveguide. This method allows for different waveguide profiles such as semi-circular guides. These structures are not evaluated in this work; however, if this method moves forward, the same simulation work on these different geometries should be performed, as they would support different modal structures.

\subsubsection{Optical Testing Parameters}

After fabrication, waveguides are tested for optical losses that result from material and structural properties. For example, losses are seen in a 90 degree turn in the waveguide because of the change in shape of the guiding structure and some modes are no longer confined. Experimental data has been gathered on different radius turns and other varied structures that will be useful for implementation of on-board optical waveguides in the future.

In the experimental test setup, a single mode fiber $(5.6 \mu \mathrm{m})$, transmitting light at the near infrared wavelength of $850 \mathrm{~nm}$, is used as an input to the multimode onboard waveguide. This single mode fiber is fixed to a micro-positioning stage and manually adjusted to maximize power throughput in the system. A second micropositioning stage with a multimode fiber connected to a power meter is placed at the opposite end of the on-board waveguide to collect the light passing through the board. This setup can be seen in Figure 2.13. Some of the simulations discussed 
later use such a single mode input as the simulation input, in order to replicate the experimental measurements.

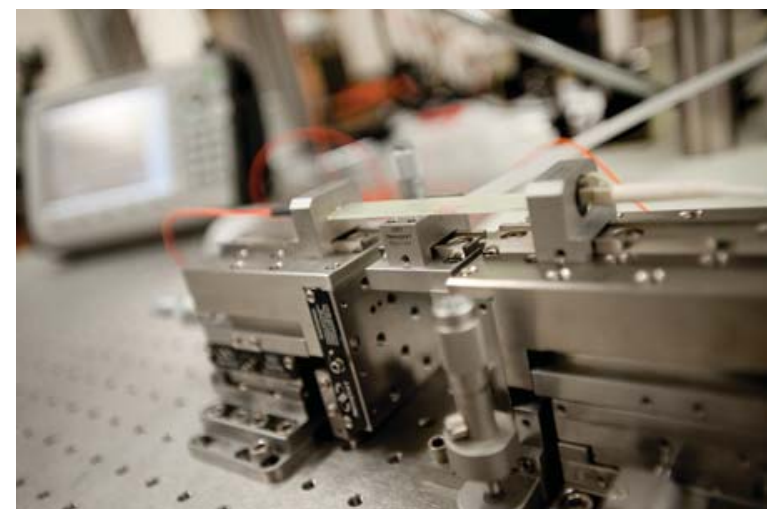

Figure 2.13: Optical test setup

\subsection{Vertical Interconnect Assembly}

In multilayer electronic circuit boards, individual layers are connected by holes in the board that are filled or electroplated with a conductor. These structures are known as vertical interconnect assemblies, or vias. This out of plane transmission is required for optical printed wiring boards as well in order to connect the board to board level devices and other boards. The target losses for the project's link budget are less than $3 \mathrm{~dB}$ per via.

The goal of this research is to characterize losses incurred in propagation through an optical via. The via was designed and fabricated by other project members. It consists of a PMMA block with a 45 degree cut made on the rear surface. Two perpendicular holes are drilled into the piece through the 45 degree cut. A temporary backing is placed on the backside of the piece while the two holes are filled with polysiloxane core material and then lens are attached to the ends of the channels. After the core material is cured and the temporary backing is removed, the 45 degree surface is coated with gold to act as a reflective surface.

In each block, twelve vias are drilled at $250 \mu \mathrm{m}$ spacing, but only six are currently used due to the lens size. Two alignment pin holes are added to the block so that it can be mated with a standard MT style connector. A schematic of the device is shown in Figure 2.14. 

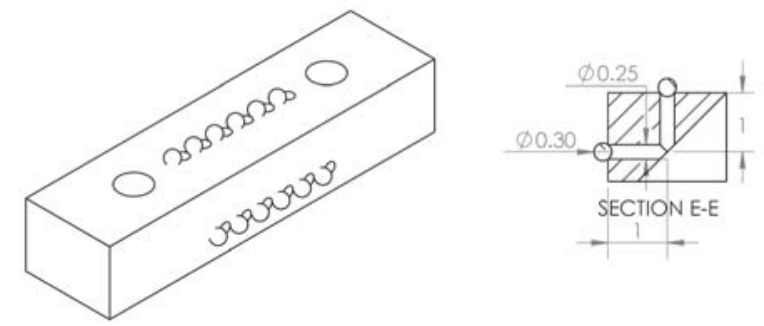

Figure 2.14: Optical via schematic

\subsection{Software Packages and Mathematical Methods}

This research deals with simulation and modeling of polymer optical waveguides and a unique optical component: the optical via. The different pieces of software and their relevant features are briefly described in the following sections.

\subsubsection{RSoft}

RSoft is an application suite that provides a variety of different tools for different types of optical modeling and simulation. Components are defined in a CAD program and different simulation modules can be applied to the structure. The two modules that will be discussed in this thesis are BeamPROP and FullWAVE.

\subsubsection{BeamPROP and BPM}

RSoft's BeamPROP is an implementation of the beam propagation method (BPM). The beam propagation method uses a finite difference solution to the Helmholtz equation, given in Eq. 2.28, to find field distributions in a waveguiding structure.

$$
\left(\nabla^{2}+k^{2}\right) \psi=\frac{\partial^{2} \psi}{\partial x^{2}}+\frac{\partial^{2} \psi}{\partial y^{2}}+\frac{\partial^{2} \psi}{\partial z^{2}}+k^{2} \psi=0
$$

Helmholtz Equation

Where, $k(x, y, z)=\frac{2 \pi}{\lambda_{0}} n(x, y, z)$ and $n(x, y, z)$ is the refractive index as a function of space. We can then define $\psi$ in terms of the field distribution and a phase factor in Eq. 2.29.

$$
\psi(x, y, z)=u(x, y, z) e^{j \bar{k} z}
$$

Eq. 2.29 Amplitude and Phase of a Wave

Where $\bar{k}$ is a constant describing the average phase change of $\psi$ in $z$. If Eq. 2.29 is plugged into Eq. 2.28, the following steps may be made. 


$$
\begin{gathered}
e^{j \bar{k} z} \frac{\partial^{2} u}{\partial x^{2}}+e^{j \bar{k} z} \frac{\partial^{2} u}{\partial y^{2}}+\frac{\partial^{2} u e^{j \bar{k} z}}{\partial z^{2}}+k^{2} u e^{j \bar{k} z}=0 \\
\frac{\partial^{2} \psi}{\partial z^{2}}=e^{j \bar{k} z}\left(2 j \bar{k} \frac{\partial u}{\partial z}+\frac{\partial^{2} u}{\partial z^{2}}-\bar{k}^{2} u\right) \\
e^{j \bar{k} z}\left(\frac{\partial^{2} u}{\partial x^{2}}+\frac{\partial^{2} u}{\partial y^{2}}+2 j \bar{k} \frac{\partial u}{\partial z}+\frac{\partial^{2} u}{\partial z^{2}}-\bar{k}^{2} u+k^{2} u\right)=0
\end{gathered}
$$

Eq. 2.30

Eq. 2.28 into Eq.

Eq. 2.32

Expanded Helmholtz

If we then make the following slow varying envelope approximation [12], the fundamental equation to be used for the beam propagation method in Eq. 2.33 comes out.

$$
\begin{gathered}
\frac{\partial^{2} u}{\partial z^{2}} \ll 2 j \bar{k} \frac{\partial u}{\partial z} \\
\frac{\partial u}{\partial z}=\frac{j}{2 \bar{k}}\left(\frac{\partial^{2} u}{\partial x^{2}}+\frac{\partial^{2} u}{\partial y^{2}}+\left(k^{2}-\bar{k}^{2}\right) u\right)
\end{gathered}
$$
BPM Equation

The result of this approximation is a paraxiality requirement. The approximation is valid only if the beam is travelling very closely to the $z$-axis. The field structure in $x, y$ is defined initially and subsequent values are calculated using discrete methods.

To remove the paraxiality requirement, a method known as wide-angle BPM is employed. This starts from Eq. 2.32 and replaces all $\frac{\partial}{\partial z}$ with $D$. The following quadratic equation results.

$$
\begin{gathered}
D^{2}+D(2 j \bar{k})+P \bar{k}^{2}=0 \\
P=\frac{1}{\bar{k}^{2}}\left(\frac{\partial^{2}}{\partial x^{2}}+\frac{\partial^{2}}{\partial y^{2}}+\left(k^{2}-\bar{k}^{2}\right)\right)
\end{gathered}
$$

Eq. 2.34

Quadratic form of Eq.

Eq. 2.35 P operator constant

Using the quadratic formula, the following expression is found for $D$,

$$
\begin{array}{cr}
D=j \bar{k}(\sqrt{1+P}-1) & \text { Eq. } 2.36 \\
\frac{\partial u}{\partial z}=D u=j \bar{k}(\sqrt{1+P}-1) u & \partial u / \partial z \text { using } D \text { operator }
\end{array}
$$

If a first order Taylor (Maclaurin) series expansion is used to evaluate $\sqrt{1+P}$, so that $\sqrt{1+P} \approx 1+\frac{P}{2}$, the original BPM equation found in Eq. 2.33 results. The higher order expansion employed in the method, the more accurate results will be [13]. More 
commonly, a Padé approximant is used for this step. Padé approximants are defined by a ratio of two power series. The different approximants are defined by indices which denote the order of the numerator and denominator series, respectively. The Padé order $(1,0)$ corresponds to the Maclaurin series previously mentioned [14]. As with the Maclaurin series expansion, the higher order approximation is made, the better the approximation is. RSoft allows use of Padé orders $(1,0),(1,1),(2,2)$, $(3,3)$, and $(4,4)$. As the quality of the approximation increases, the paraxiality requirement is loosened [15].

\subsubsection{FulIWAVE and FDTD}

FullWAVE is RSoft's implementation of the finite-difference time-domain method for solving electromagnetic problems. This method involves a full solution to Maxwell's equations over a defined grid of points. The fundamental concepts behind this method are discussed below.

Beginning with Faraday's law, the following derivation is performed.

$$
\begin{aligned}
& \nabla \times \boldsymbol{E}=-\frac{\partial \boldsymbol{B}}{\partial t} \\
& \nabla \times \boldsymbol{E}=\left|\begin{array}{ccc}
\hat{x} & \hat{y} & \hat{z} \\
\frac{\partial}{\partial x} & \frac{\partial}{\partial y} & \frac{\partial}{\partial z} \\
E_{x} & E_{y} & E_{z}
\end{array}\right|= \\
& \hat{x}\left(\frac{\partial E_{z}}{\partial y}-\frac{\partial E_{y}}{\partial z}\right)-\hat{y}\left(\frac{\partial E_{z}}{\partial x}-\frac{\partial E_{x}}{\partial z}\right)+\hat{z}\left(\frac{\partial E_{y}}{\partial x}-\frac{\partial E_{x}}{\partial y}\right)= \\
& -\left(\hat{x} \frac{\partial B_{x}}{\partial t}+\hat{y} \frac{\partial B_{y}}{\partial t}+\hat{z} \frac{\partial B_{z}}{\partial t}\right)
\end{aligned}
$$

This equation can be broken into three separate scalar equations as follows.

$$
\begin{array}{rr}
-\frac{\partial B_{x}}{\partial t}=\frac{\partial E_{z}}{\partial y}-\frac{\partial E_{y}}{\partial z} & \text { Eq. 2.39 } \\
-\frac{\partial B_{y}}{\partial t}=\frac{\partial E_{x}}{\partial z}-\frac{\partial E_{z}}{\partial x} & \text { Scalar Maxwell's Equation } 1 \\
-\frac{\partial B_{z}}{\partial t}=\frac{\partial E_{y}}{\partial x}-\frac{\partial E_{x}}{\partial y} & \text { Eq. 2.40 } \\
\text { Scalar Maxwell's Equation 2 }
\end{array}
$$

The same thing can be done starting with Ampère's Law to yield equations for the components of $\frac{\partial E}{\partial t}$, giving a total of six equations. 
A grid of spatial points must then be defined with spacing $\Delta x, \Delta y$, and $\Delta z$. The values of $\boldsymbol{E}$ and $\boldsymbol{B}$ can then be calculated across the entire grid from the initial conditions after a defined time step $\Delta t$. In order to avoid solving for both $\boldsymbol{E}$ and $\boldsymbol{B}$ simultaneously, the grids for $\boldsymbol{E}$ and $\boldsymbol{B}$ are offset by $\frac{\Delta x}{2}, \frac{\Delta y}{2}$, and $\frac{\Delta z}{2}$. This is an acceptable approximation as long as Eq. 2.42 is held where $c_{\max }$ is the maximum speed of light in the medium.

$$
\sqrt{\Delta x^{2}+\Delta y^{2}+\Delta z^{2}}>c_{\max } \Delta t
$$

FDTD Time Step Requirement

The actual calculations for $\boldsymbol{E}$ and $\boldsymbol{B}$ components are made using Eq. 2.43, where $n$ is the time step index and $i, j, k$ are the grid points discussed above.

$$
\begin{aligned}
& \frac{B_{x}^{n+1 / 2}(i, j+1 / 2, k+1 / 2)-B_{x}^{n-1 / 2}(i, j+1 / 2, k+1 / 2)}{\Delta t} \\
= & \frac{E_{y}^{n}(i, j+1 / 2, k+1)-E_{y}^{n}(i, j+1 / 2, k)}{\Delta z} \\
- & \frac{E_{z}^{n}(i, j+1, k+1 / 2)-E_{z}^{n}(i, j, k+1 / 2)}{\Delta y}
\end{aligned}
$$

Finite Difference

Analogous equations can be defined for each of the six scalar Maxwell's equations. Each calculation is performed at every grid point for both $\boldsymbol{E}$ and $\boldsymbol{B}$ in the simulation domain to find the field values [16]. This can be very time consuming since these equations have to be calculated at every point in the simulation region.

\subsubsection{Simulation Launch Options}

The two methods of input used in this work are RSoft's slab mode and multimode. Slab mode corresponds to the fundamental mode of the launch structure. A multimode input is created by first calculating all the supported modes of the launch structure. Each of these modes is given a random phase and then equally weighted and summed together.

\subsubsection{ZEMAX}

ZEMAX is used for optical system modeling and design. It does not perform the same types of calculations as RSoft and uses very different methods of calculation. Generally, ZEMAX is used for ray tracing, which is part of the geometrical approximation of optics, and is done by assuming an infinitely small wavelength. There is an exception to this in the Physical Optics Propagation (POP) module within ZEMAX. This is the portion of the software that will be used in this thesis. 


\subsubsection{Physical Optics Propagation}

The ZEMAX physical optics propagation has two methods of propagation; angular spectrum propagation and Fresnel propagation. Which method is used is determined by the Fresnel number, given in Eq. 2.44, where $A$ is the beam radius and $Z$ is the propagation distance [17], [18].

$$
F_{n}=\frac{A^{2}}{\lambda Z}
$$

Fresnel

Number

For $F_{n}<1$, the propagation is said to be in the far field, for which ZEMAX uses the Fresnel propagator and while $F_{n}>1$, near field angular spectrum propagation is used. ZEMAX allows the user to override this automatic selection and select the angular spectrum propagator as well. The regions where angular spectrum and Fresnel propagations are appropriate are shown in Figure 2.15.

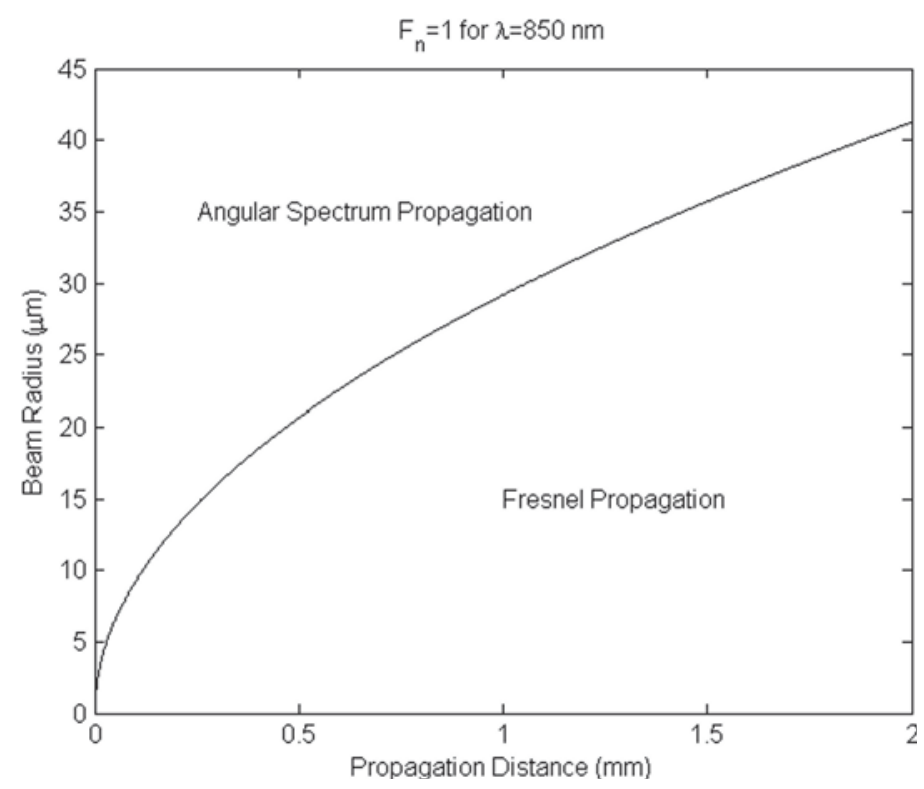

Figure 2.15: Propagation method selection based on Fresnel number

For this work, the Fresnel number will be larger than one, so the angular spectrum propagation will be used by ZEMAX in its calculations. The reasoning behind this assertion will be discussed in section 3.4.

\subsubsection{ZEMAX Programming Language}

ZEMAX has a built in macro language called ZEMAX Programming Language, or ZPL. This tool allows the user to automate different ZEMAX tasks such as changing parameters and executing simulations. It is similar to BASIC and can be quickly 
understood. The ZEMAX User's Manual contains a full description of available functions and syntax [17].

The macro files are written in a text editor outside of ZEMAX and saved to the ZEMAX macros directory. The macro is then run by selecting it from the macros menu in ZEMAX. The code used for this project is included in the appendix.

\subsubsection{MATLAB}

MATLAB is used as a general tool throughout this work due to the author's familiarity with it and its ease of use in many areas. Relevant code will be included in the appendix and the functions used for various tasks will be mentioned in context. This section includes background on mathematical concepts dealt with in the MATLAB portions of this work.

\subsubsection{I mage Cross Correlation}

The cross correlation of two images indicates how similar the images are. The maximum value indicates the position at which the second image best matches the first image [19]. Using the MATLAB function xcorr2, the cross correlation of two images can easily be computed. This concept will be used to find repeating mode structures in a waveguide. Two cross sections can be cross correlated and, from the resulting function, their similarity can be found. If the maximum occurs at the center of the matrix, the two images are most similar when overlaid on top of each other, indicating a repeating mode structure. 



\section{Chapter 3}

\section{Model Development}

\subsection{Beam propagation method (RSoft BeamPROP)}

The Beam Propagation Method (BPM) is a popular method for waveguide modeling based on its relative simplicity. Its ease of implementation for a particular problem paired with its relatively short run times make it an efficient choice for modeling waveguide systems [20].

The main problem in using BPM for modeling of a via system is the paraxiality requirements [21]. Paraxiality conditions are met when light travels in a direction with a small angle difference to the optical axis. In a via system, light is expected to travel at $90^{\circ}$ to the original optical axis. Some BPM methods have been developed to account for this [22]; however they are not available in RSoft's BeamPROP software.

By using Padé approximations, the BPM method can be improved for wide-angle propagations [15]. Due to the simplicity of use of the BeamPROP software, a quick analysis can be made using the different Padé approximants to see the effects of changing this approximation. Some parameters of these simulations are in Table 3.1 .

Table 3.1

Padé approximant simulation parameters

\begin{tabular}{|l|l|}
\hline Core index & 1.53 \\
\hline Cladding index & 1.51 \\
\hline Wavelength & $850 \mathrm{~nm}$ \\
\hline Core width & $50 \mu \mathrm{m}$ \\
\hline Reflector material & Gold \\
\hline
\end{tabular}

First, a straight waveguide of length $500 \mu \mathrm{m}$ was created which intersected another straight waveguide creating a $90^{\circ}$ angle. A gold reflector was placed at $45^{\circ}$ in the intersection using the built in RSoft gold profile. The simulation was run with each available Padé order. The beam propagated correctly through the initial waveguide, however did not reflect as desired at the gold interface for each Padé order. The results of the lowest and highest Padé orders can be seen in Figure 3.1 and Figure 3.2 , respectively. Next, the system was tilted $45^{\circ}$ to minimize the required change in direction with respect to the z-axis. The launch angle was tilted at $45^{\circ}$ as well. As 
can be seen in Figure 3.3 and Figure 3.4, the simulation was unable to guide the wave through the first section at all.

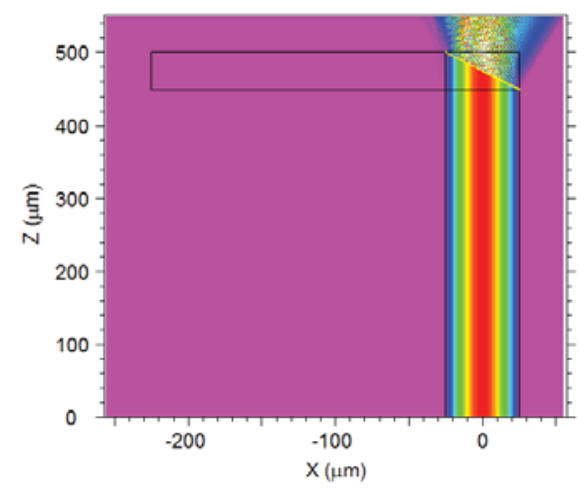

Figure 3.1: Straight $90^{\circ}$ Turn with Padé order $(1,0)$
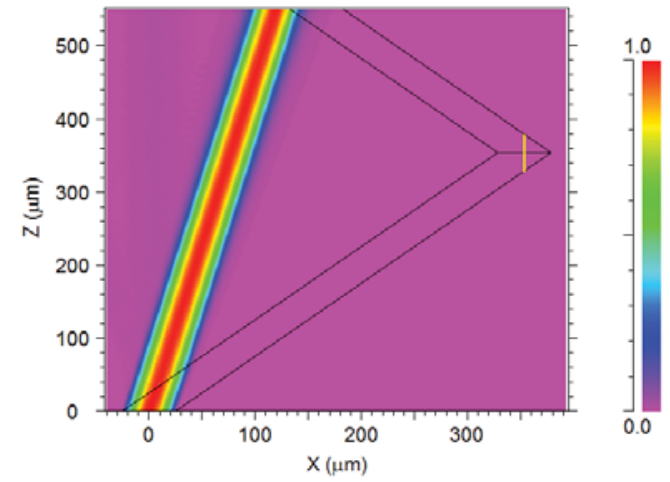

Figure 3.3: Tilted $90^{\circ}$ turn with Padé order $(1,0)$

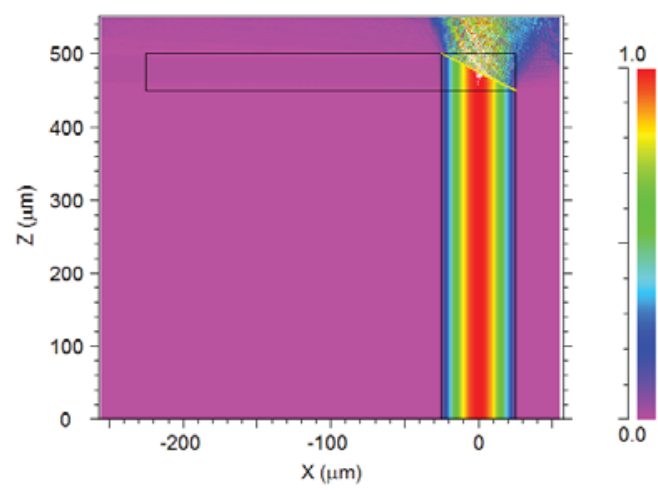

Figure 3.2: Straight $90^{\circ}$ turn with Padé order $(4,4)$

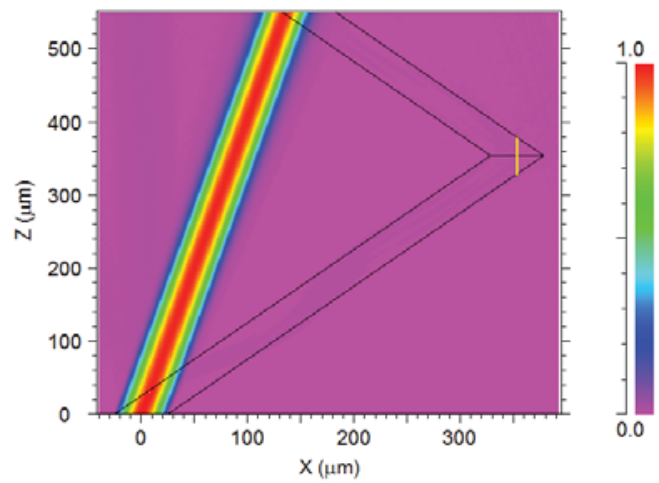

Figure 3.4: Tilted $90^{\circ}$ turn with Padé order $(4,4)$

These results quickly and clearly indicated that modeling a via system in BeamPROP would not provide any acceptable results. The BPM was not entirely discounted, however, and its application in this work will be further discussed in a later section.

\subsection{Finite difference time domain method (RSoft FullWAVE)}

The Finite difference time domain method (FDTD) implemented in RSoft's FullWAVE software offered the opportunity to model the same system described in the previous subsection using a full solution to Maxwell's equations. This would allow propagation to remain in the waveguide structure and correctly reflect off of the gold surface. This is demonstrated in a small scale simulation in Figure 3.5. 


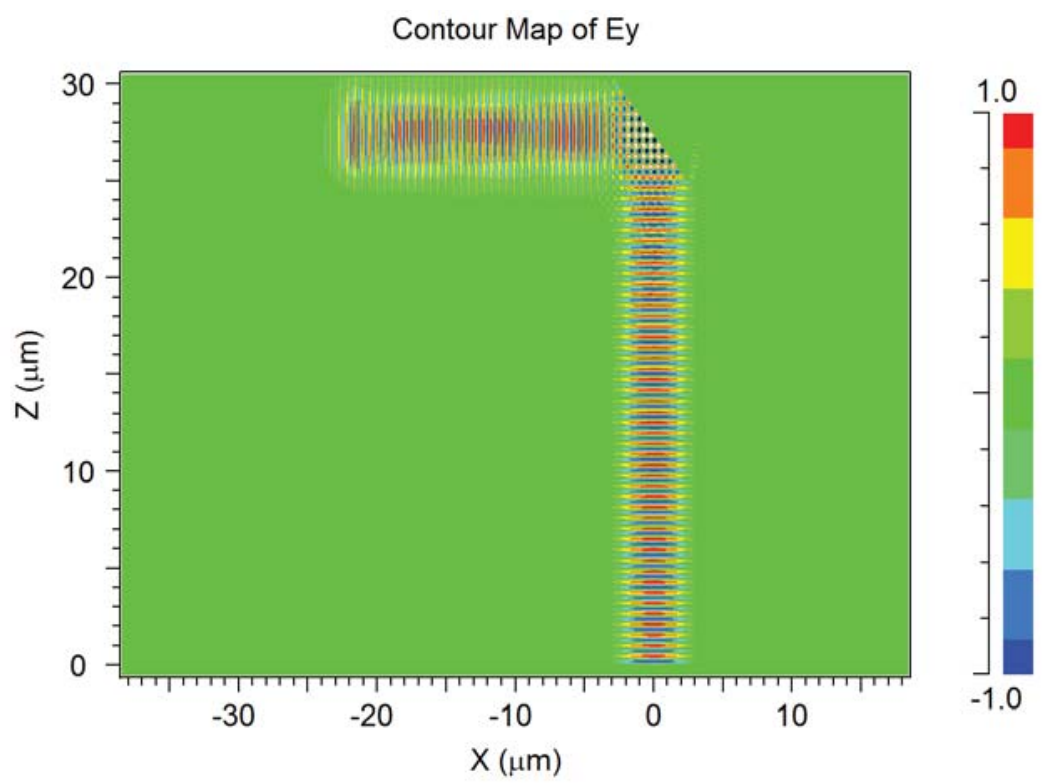

\section{Figure 3.5: FullWAVE simulation of straight $90^{\circ}$ turn}

Using the same CAD files from the previous subsection in the Padé order investigation, FullWAVE simulation requirements were determined based on simulation region grid size. For the untilted simulation, each time step of the simulation would require 1.74 seconds and over 2800 MB of memory, while the tilted simulation would require 2.46 seconds and over 4000 MB of memory, despite its only slightly larger simulation area. Each time step represents .025*cT (default value), which corresponds to about $12.5 \mathrm{~nm}$ of propagation length. To propagate the length of the first waveguide leg $(500 \mu \mathrm{m})$, this requires 40,000 time steps, or 19.3 hours. Bearing in mind that this is only half of an atypically small simulation, it was argued that this approach is also not acceptable.

The root of the problem in using FDTD for this sort of simulation is the requirement that every point on a rectangular grid be calculated for. For this reason, the method does not do well as the simulation size scales up [23]. As the length of each leg increases, the empty space in the lower left corner of Figure 3.5 increases quickly.

Attempts were made to use the non-uniform grid feature in FullWAVE, however the non-uniform grid was only allowed to be defined at interfaces (the edges of the guide). This leads to acceptable sampling along the edges and inadequate sampling in the middle of the waveguide (which is still of interest) along with the desired low sampling of the empty space. Use of parallel processing with GPUs, for example, was briefly discussed, but not pursued. 


\subsection{Ray Tracing (ZEMAX)}

A simple ZEMAX model was designed in sequential ray tracing mode. This model can be seen in Figure 3.6. This model uses ray tracing techniques, which is unable to describe the behavior of the system with different modal structures used as inputs. It provides a nice geometric visual of what's going on in the system, but is unable to provide any real simulation data.

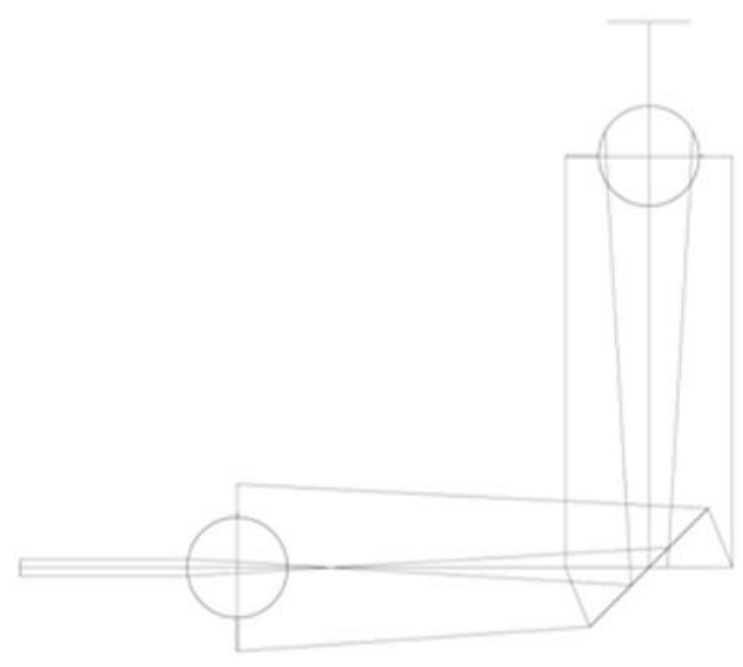

Figure 3.6: Simple ZEMAX ray tracing model

\subsection{Physical Optics Propagation (ZEMAX)}

The physical optics module in ZEMAX allows for the propagation of field data through a system using the angular spectrum or a Fresnel propagator. The same model that was developed using the ray tracing technique can be used with the POP module. ZEMAX has some field profiles packaged with the software; however it is unable to generate these varied fields itself.

In the ray diagram pictured in Figure 3.6, it can be seen that the beam is not incident on a surface with notably lower beam size than the original $50 \mu \mathrm{m} \times 50 \mu \mathrm{m}$. This fact, in combination with the propagation distances between surfaces all above $0.15 \mathrm{~mm}$ indicate that the angular spectrum is the correct choice for propagation technique based on the Fresnel number analysis shown in Figure 2.15.

\subsection{Multi-software Approach}

None of these single software approaches led to efficient results; some of them not even providing acceptable results. It was then decided to use the strengths of each piece of software and combine them to produce efficient results. RSoft is able to 
provide modal information from light propagating in a waveguide, but does not do well with direction of propagation changes. On the other hand, ZEMAX does not do electromagnetic mode solving, but is able to propagate definable inputs through complicated optical systems. It was decided that a good solution would be to use RSoft to generate modal structures which would then be input into ZEMAX for propagation through the via.

\subsubsection{RSoft mode generation}

RSoft's BeamPROP software is able to provide cross-sectional slices of the field within a waveguide. The spacing of these cross-sections is user definable in the simulation parameters. The parameters listed in Table 3.2 were used for the following simulations. Any variations will be noted in the specific sections.

Table 3.2

RSoft mode generating simulation parameters

\begin{tabular}{|l|l|}
\hline Core index & 1.53 \\
\hline Cladding index & 1.51 \\
\hline Core width & $50 \mu \mathrm{m}$ \\
\hline Core height & $50 \mu \mathrm{m}$ \\
\hline Wavelength & $850 \mathrm{~nm}$ \\
\hline Cross-section sampling & $10 \mu \mathrm{m}$ \\
\hline Launch profile & $\begin{array}{l}5.6 \mu \mathrm{m} \\
\text { SM fiber }\end{array}$ \\
\hline
\end{tabular}

The motivation for using different structures to generate modes is that the specific modes that will be present in an actual system implementation will be unknown. Each structure will provide a different insight into the performance of the via structure.

\subsubsection{1 $15 \mathrm{~mm}$ Straight - Single Mode Fiber I nput}

A square waveguide of length $15 \mathrm{~mm}$ was defined to investigate the repeating mode structures in this symmetric guide profile. This length was chosen to allow a full repetition of modes in the off center single mode fiber input. Two different inputs were used in order to generate different mode structures in the waveguide.

\section{Centered single mode fiber input}

This set of modes was generated by inputting a $5.6 \mu \mathrm{m}$ single mode fiber at the center of the waveguide as seen in Figure 3.7.

2. Off center single mode fiber input

This set of modes was generated with the $5.6 \mu \mathrm{m}$ single mode fiber input in the center of the upper right quadrant of the waveguide as seen in Figure 3.8. 


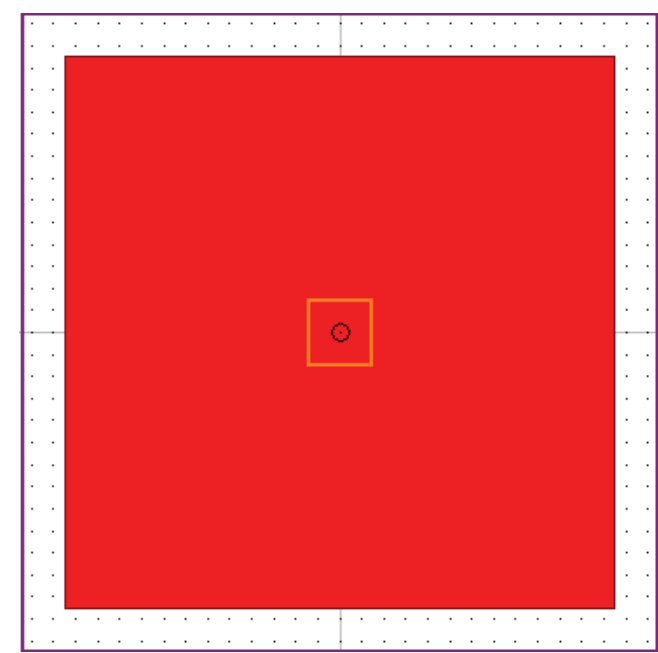

Figure 3.7: Centered single mode input

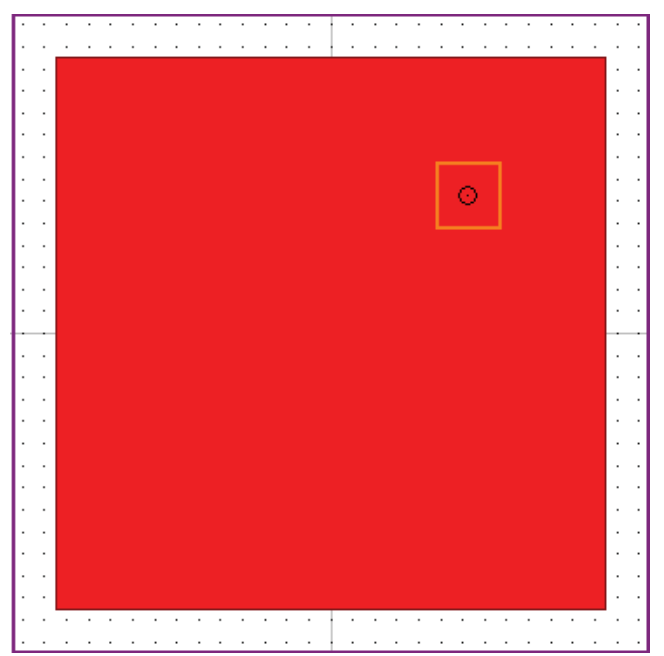

Figure 3.8: Off center single mode input

\subsubsection{2 $15 \mathrm{~mm}$ Straight - Multimode I nput}

This set of modes was generated using RSoft's built in multimode launch conditions. The launch profile filled the entire waveguide cross section so that all modes supported by the structure would be filled. The same file was used as in the other straights with only the launch conditions changed.

\subsubsection{ZEMAX Via Model}

The ZEMAX model for the via consists of a free space propagation distance, two 300 $\mu \mathrm{m}$ BK7 ball lenses, two volumes of BK6 (for core material) and a reflecting mirror. The light enters through one ball lens and exits through the other as seen in Figure 3.9.

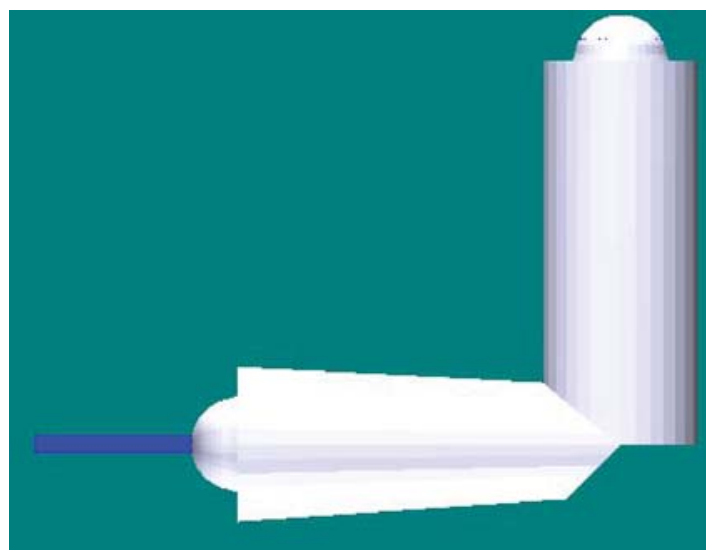

Figure 3.9: ZEMAX via model layout 
According to the ZEMAX Schott glass catalog, the index of refraction of BK6 is 1.5311 and transmission at $850 \mathrm{~nm}$ over $25 \mathrm{~mm}$ to be $99.5 \%$, which corresponds of a .0087 $\mathrm{dB} / \mathrm{cm}$ loss. Since the core material in use has an index of refraction of 1.53 and approximately $.04 \mathrm{~dB} / \mathrm{cm}$ loss, the fill material in the via is modeled using BK6. There will be higher absorption in the real system; however the propagation through this material is less than $2 \mathrm{~mm}$ total, so the difference in results is insignificant.

The object plane is designed to use a file input (taken from the RSoft mode generation) and the Physical Optics Propagation (POP) method is used to propagate that input to the image plane (on top in Figure 3.9). At this point, ZEMAX outputs a ZEMAX beam file (ZBF) that can be used for further analysis. 



\section{Chapter 4}

\section{Running the Simulation}

\subsection{RSoft parameters}

The cross sectional grid in the BeamPROP simulation was defined to cover the region from $-28.8 \mu \mathrm{m}$ to $28.8 \mu \mathrm{m}$ at $50 \mathrm{~nm}$ spacing between points. This covers the entire $50 \mu \mathrm{m} \times 50 \mu \mathrm{m}$ waveguide with some space around the edges. The simulation was run from 0 to $15 \mathrm{~mm}$ in the $z$ direction, saving a cross section slice every $10 \mu \mathrm{m}$. No parameters for the input power were set. Power normalization was taken care of in a different step of the process.

\subsection{RSoft output}

RSoft outputs files based on the prefix defined by the user and then sequentially numbers every file starting at 0 . For this reason, a multiple of ten was chosen for the cross-section sampling distance $(10 \mu \mathrm{m})$. For example, if the selected prefix is 15mm_sm_straight, 15mm_sm_straight. 000 corresponds to the slice at $z=0$ in the RSoft simulation. 15mm_sm_straight.001 would then correspond to the slice taken at $z=10 \mu \mathrm{m}$ and so on. This simple numbering scheme makes accessing the files a simple matter of counting (using a for loop). The values stored in these files are in ASCII format with standard delimiters, so they can be viewed easily with a text editor or spreadsheet tool; however they may be quite large.

\subsection{RSoft to ZEMAX conversion}

Built in to RSoft is a Raytracing File Converter utility. This utility allows the user to convert between RSoft and ZEMAX or Code $V$ file formats. There is a simple graphical user interface (GUI) for this utility; however there is no command line interface (CLI) command detailed in the user's guide. CLI options are listed for Code $V$ conversion, but not ZEMAX. It was suspected that, as with most RSoft processes, a CLI option was available, but for some reason not referenced in the user's guide. Through contact with an RSoft support engineer, it was initially determined that this functionality was not available, but after further discussion with a developer, it was available, but not documented. An addition to the user's manual will be added in future editions and this addition was made available for this project. It is included in the appendix as well.

The CLI functionality was used in MATLAB using the system function, where MATLAB issues system commands. Due to the convenient naming scheme for RSoft output 
files, a quick script can easily be written to loop through the appropriate numbers and issue a command to the RSoft Raytracing File Converter. This method was used on all RSoft output files. ZEMAX output files were 1024 x 1024 arrays of complex field values. The naming scheme for these files was modified slightly, but retained both the prefix and the numbering of the original RSoft files. Each file was named with the following format: [prefix][number].zbf.

One advantage of this processing step is the decrease in file size between RSoft and ZEMAX file formats. RSoft stores its data as ASCII (text) values, whereas ZEMAX stores all values in binary, allowing for a smaller file size and quicker read times for the same data. Any later manipulation of field data was done using the ZEMAX file format for this reason.

\subsection{Mode Profiles and Repeating Structures}

After the mode files were converted to the ZEMAX file format, the beams were read into MATLAB to create a cross sectional view of the length of the waveguide. The field inside the waveguide was summed over one axis to create a one dimensional cross section. Each one dimensional cross section was then used as a row in an image to create the mode profile with respect to the $z$ axis (direction of propagation).

During this process, the cross correlation of each slice with the first slice was computed. The maximum value of the cross correlation corresponds to the best possible match of the two images, as previously mentioned [19]. From the large resulting cross correlation matrix, two values were taken; the value at the center of the matrix and the maximum value. The value at the midpoint indicates the similarity when the two images are laid directly on top of each other. The maximum value should be the midpoint if the images are the same. It is also possible that the midpoint is not the maximum. The meaning of this will be discussed later. To save on computation time, the matrices that were cross correlated were shrunk by a factor of four, using the imresize function with box interpolation in MATLAB.

The goal of this step is to determine if the mode structure is repeating. This allows steps to be taken to ensure the inputs are not repeated, which would unequally weight the input structures and potentially skew the final output data. Additionally, images of the modal structure along the propagation length are easily generated within this code.

\subsection{ZEMAX macro}

The ZEMAX macro allows the same physical optics propagation to run iteratively, changing the beam input for each iteration. The macro is run from within ZEMAX by selecting it from the Macros menu. The user is then asked for a file prefix, start 
number, end number, and to which surface the input should be propagated. The file prefix corresponds to prefix used in the previously mentioned file naming format and the start and end numbers correspond to the range of numbers to loop through propagating each corresponding input file.

Using the MODIFYSETTINGS command in ZPL, the source file is set to the file corresponding to the current iteration and the total power of the input is set to one. This is meant to isolate the via effects from the waveguide structure by removing any power loss that may have occurred during the RSoft simulation. Additionally, normalizing the power to one makes it simple to translate the final output power to a percentage. The physical optics propagation is then executed and the output file is named with a new prefix that is related to the original prefix.

The macro was run manually for each set of mode inputs and each set was run twice, with the input was placed at a lead-in distance of $250 \mu \mathrm{m}$ and $500 \mu \mathrm{m}$ from the front of the first ball lens.

\subsection{MATLAB Summary of Results}

MATLAB was used to read in all the output ZEMAX files and calculate the total power present in the output. A square or circular region of interest can be defined to zero out any power outside of the desired region. If the desired region is larger than the available region in the file, a warning is given. This is useful for calculating the power present within the connector fiber radius. A circle of diameter $125 \mu \mathrm{m}$ was used in these simulations; however it did not impact the results, as effectively all of the power was within this region.

The ZEMAX ZBF data is stored so that $E_{x} * E_{x}+E_{y} * E_{y}$ is defined as watts [17]. For this reason, the matrix of values from the ZEMAX output is simply added up to calculate total power at the end of propagation. The number of inputs that provided output in a given $10 \mathrm{~mW}$ range were counted and graphed. 



\section{Chapter 5}

\section{Results}

\section{$5.115 \mathrm{~mm}$ Straight - Centered Single Mode Input}

A selection of mode cross sections using the $15 \mathrm{~mm}$ straight with centered single mode input is shown in Figure 5.1, chosen simply at $100 \mu \mathrm{m}$ spacing. It can be seen that the modal structures appear to have regular patterns, but vary greatly at different distances.

The distribution across $\mathrm{z}$ with the calculated cross correlation data is displayed in Figure 5.2. As is expected, the mode distribution can be seen to repeat at a regular interval. The presence of a repeated field distribution is verified by the cross correlation data peaks. Both the maximum and midpoint methods peak where the repetitions occur. 

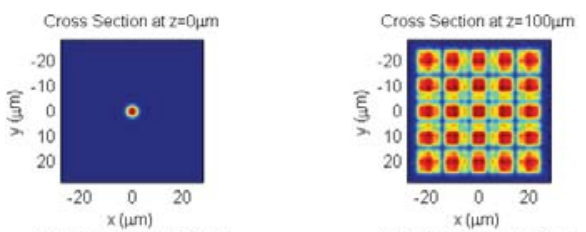

Cross Section at $z=400 \mu \mathrm{m}$
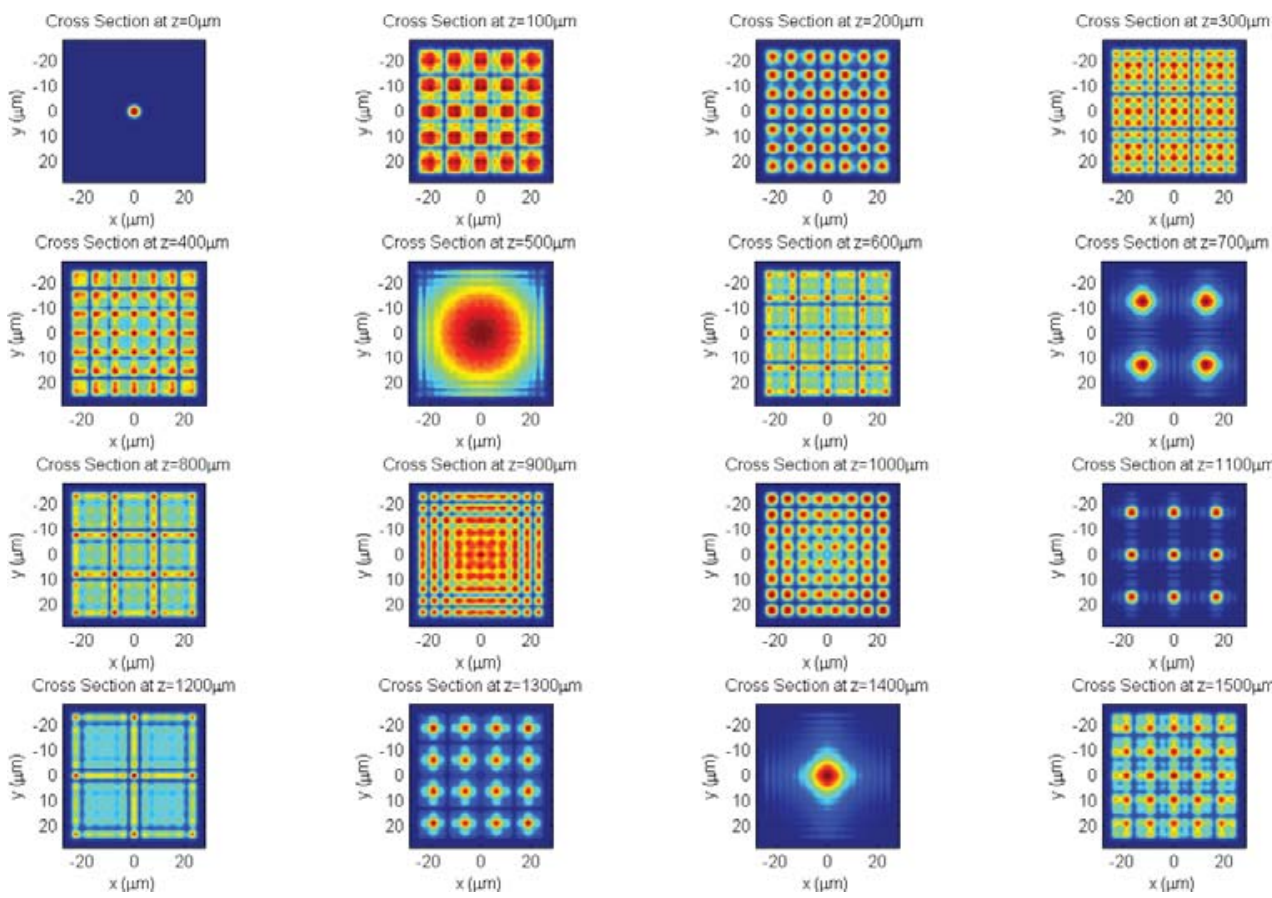

Cross Section at $z=1100$ ur

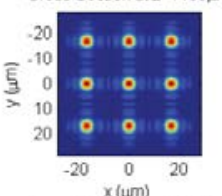

Cross Section at $z=1500$ ur

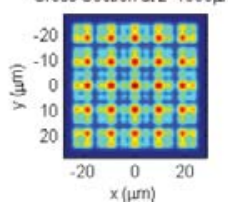

Figure 5.1: Selection of modes in $15 \mathrm{~mm}$ straight with centered single mode input
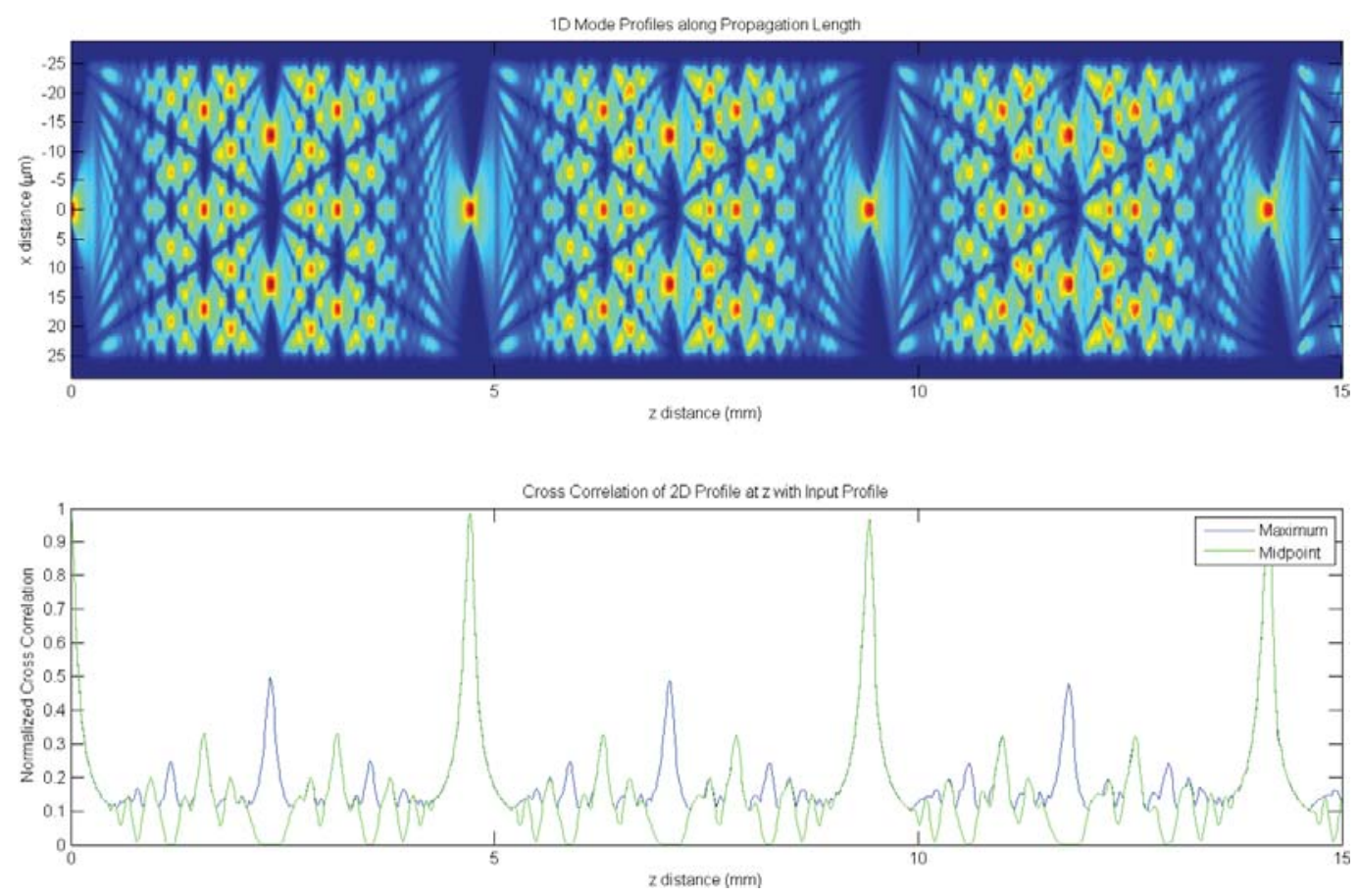

Figure 5.2: $15 \mathrm{~mm}$ straight with centered single mode input mode profile and cross correlation data 
These repetitions also illustrate the difference between the maximum and midpoint methods. Halfway between each repetition of the centered input distribution, there occurs a split in the distribution with two lobes equally spaced about the center near $z=2.5 \mathrm{~mm}$, for example. Here, the maximum cross correlation method peaks, because each of the two lobes is similarly distributed to the original central lobe; however the midpoint method shows that this maximum cross correlation does not occur when the two distributions are centered on each other.

Based on this data, the specific files to propagate through the via model can be determined. It is undesired to unequally weight the modal structures in the final results, so the inputs should be taken from an integer number of repetitions of the distributions. Since they are nearly exact replicas, it is practical to use only one cycle, corresponding to the slices between 0 and $4.70 \mathrm{~mm}$. This first section is the best choice because while the modal distribution repeats itself, there are some slight differences that develop as a result of propagation, which account for the slight decrease in cross correlation peaks as z increases.

The results from the via propagation for both lead-in distances are seen below in Figure 5.3 and Figure 5.4. The upper graph in each figure shows the power output for each mode propagation length, with the mean of these values graphed as well. The lower graph displays the number of inputs that provided a given output power, similar to an image histogram. The closer input had a higher mean power output at $47.0 \%(-3.28 \mathrm{~dB})$, while the input placed $500 \mu \mathrm{m}$ from the via had an average power output of $46.1 \%(-3.36 \mathrm{~dB})$. The peaks correspond to mode structures when the majority of the power was centrally concentrated or converging to the center. 

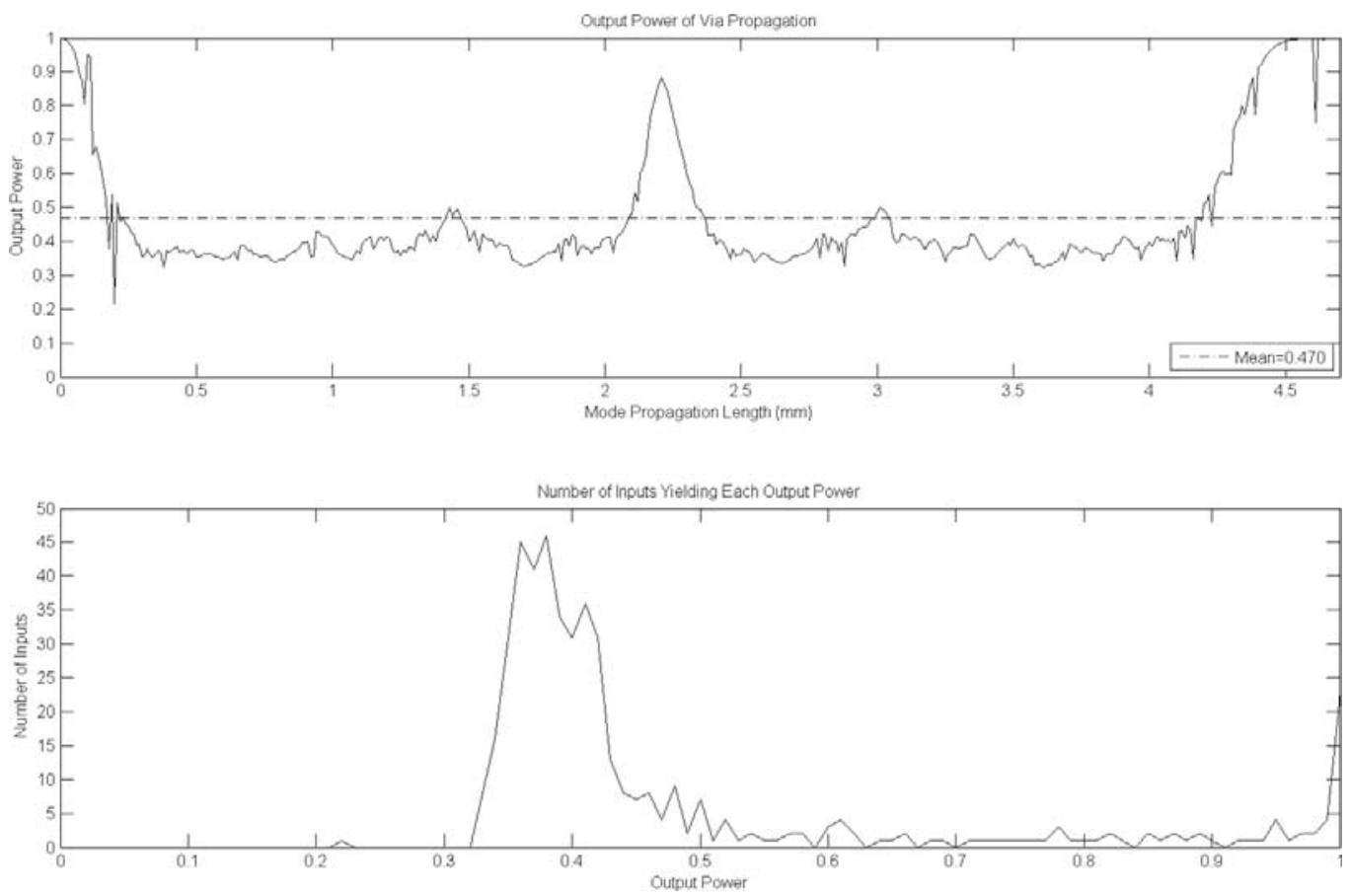

Figure 5.3: Power output after via propagation for $\mathbf{2 5 0} \boldsymbol{\mu m}$ lead-in (center SM input)
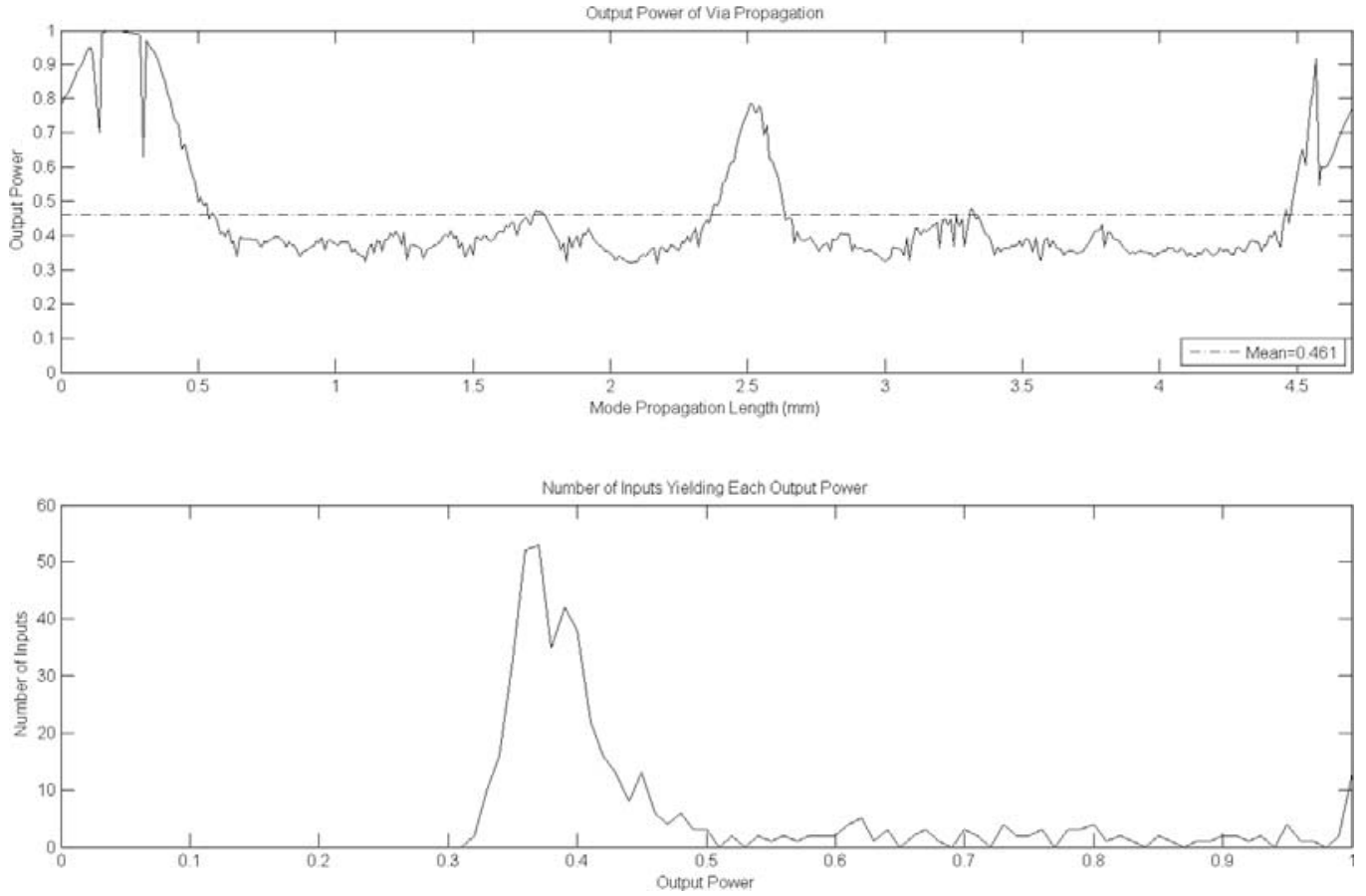

Figure 5.4: Power output after via propagation for $500 \mu \mathrm{m}$ lead-in (center SM input) 


\section{$5.215 \mathrm{~mm}$ Straight - Off Center Single Mode Input}

Again, a selection of modes at $100 \mu \mathrm{m}$ spacing, this time using the $15 \mathrm{~mm}$ straight with off center single mode input, is shown in Figure 5.5 and the corresponding mode profile and cross correlation data is shown in Figure 5.6.

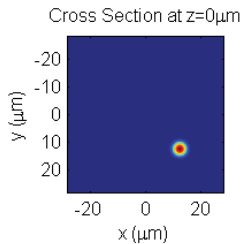

Cross Section at $z=400 \mu \mathrm{m}$

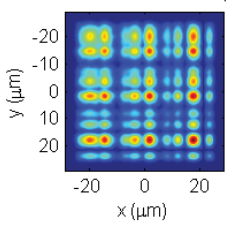

Cross Section at $z=800 \mu \mathrm{m}$

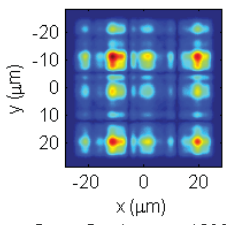

Cross Section at $z=1200 \mu \mathrm{m}$

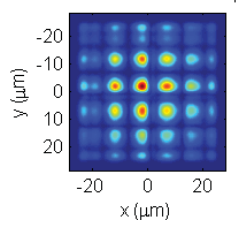

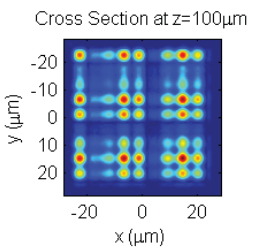

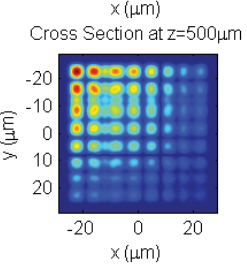

Cross Section at $z=900 \mu \mathrm{m}$

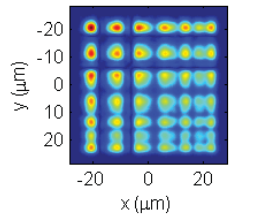

Cross Section at $z=1300 \mu m$

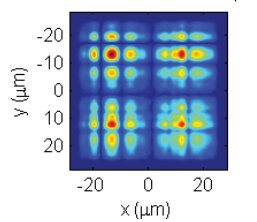

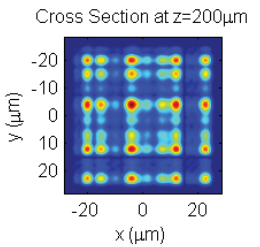

Cross Section at $z=600 \mu \mathrm{m}$

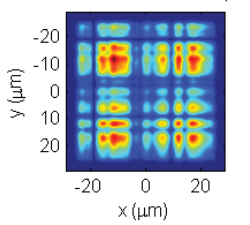

Cross Section at $z=1000 \mu \mathrm{m}$

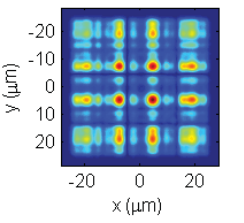

Cross Section at $z=1400 \mu \mathrm{m}$

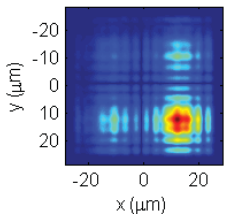

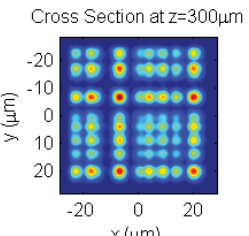

Cross Section at $z=700 \mu m$

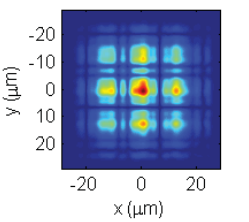

Cross Section at $z=1100 \mu m$

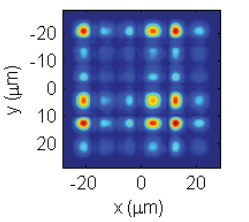

Cross Section at $\mathrm{z}=1500 \mu \mathrm{m}$

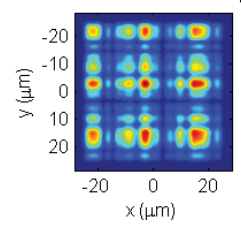

Figure 5.5: Selection of modes in $15 \mathrm{~mm}$ straight with off center single mode input 

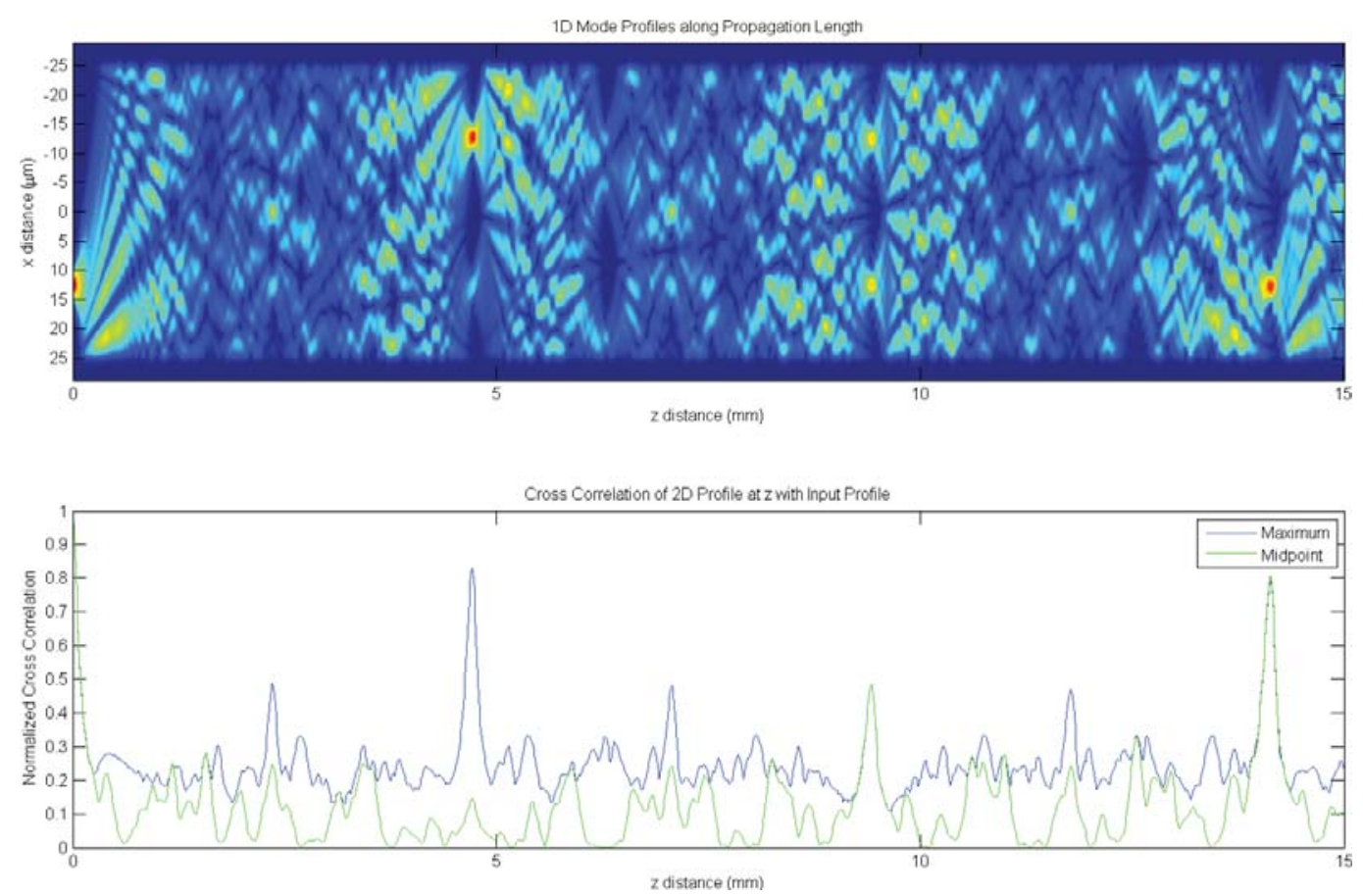

Figure 5.6: $15 \mathrm{~mm}$ straight with off center single mode input mode profile and cross correlation data
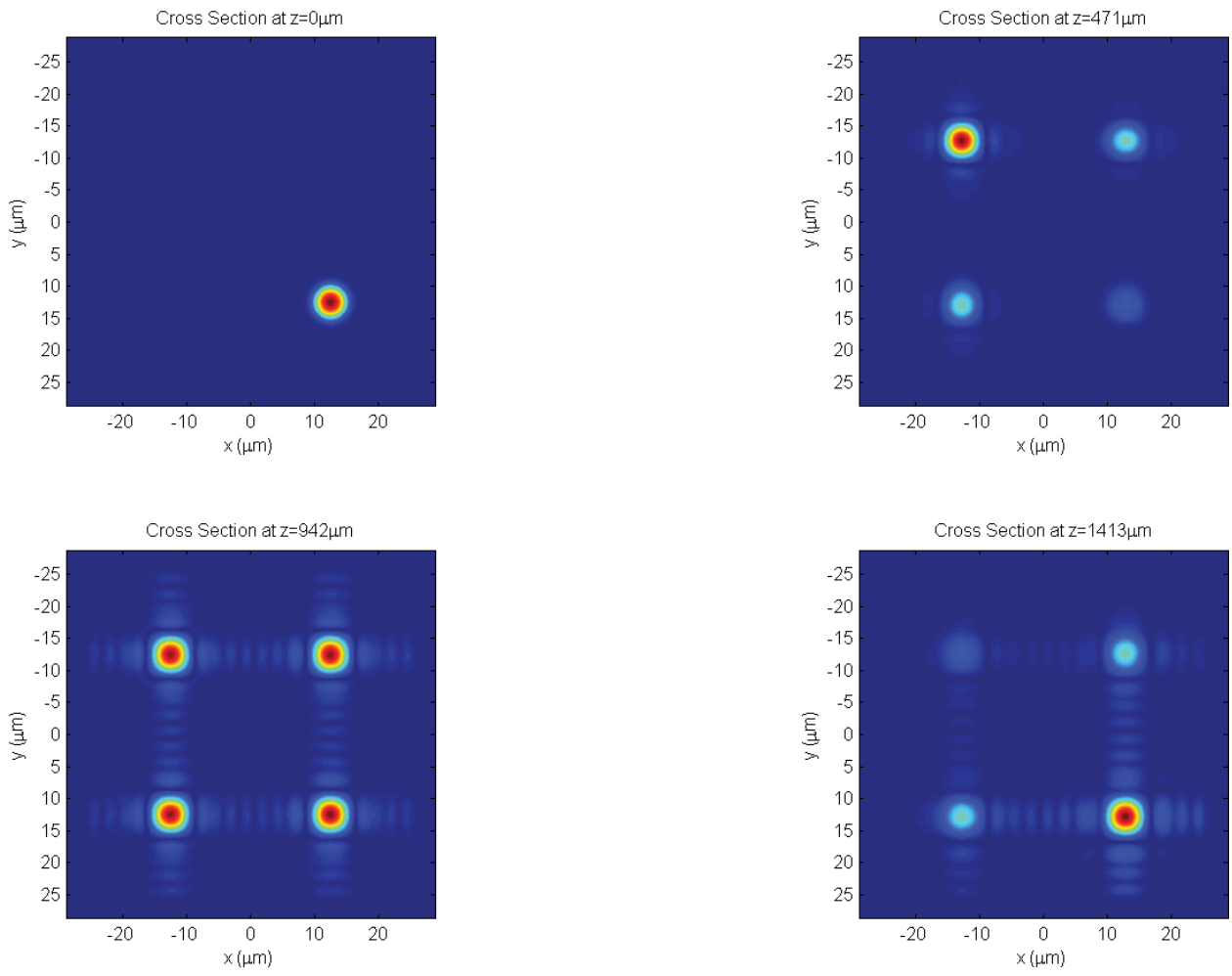

Figure 5.7: $15 \mathrm{~mm}$ straight with off center single mode input modal distributions corresponding to cross correlation peaks 
The large peak in the maximum cross correlation value occurs when the modal distribution is almost perfectly repeated, but in a different location. The small peak in both maximum and midpoint corresponds to a distribution where the input is repeated, but there are other significant components in the distribution. Finally, the major shared peak is a repetition of the original distribution; however energy has begun to be distributed away from the original point of input. Once again, this degradation in mode repetition corresponds to a lower cross correlation peak.

The power outputs of the via propagation can be seen plotted in Figure 5.8 and Figure 5.9. As in the centered input case, several peaks in the cross correlation data appear, indicating some sort of repetition in the mode structure. These major peaks occur at $4.71 \mathrm{~mm}$ and $14.13 \mathrm{~mm}$ and a secondary peak in both methods occurs at $9.42 \mathrm{~mm}$. The modal distributions at these particular positions are displayed in Figure 5.7 .

The average losses in both the $250 \mu \mathrm{m}$ and $500 \mu \mathrm{m}$ lead-in were equal at $45.3 \%$ throughput or $-3.44 \mathrm{~dB}$. Again, the peaks corresponded to the mode structures where the power is concentrated in a small area. The wider peaks corresponded with the locations that this concentrated power was in the center of the waveguide. The skinnier peaks correspond to the modal structures where the power was concentrated in a small region which is not at the center. 

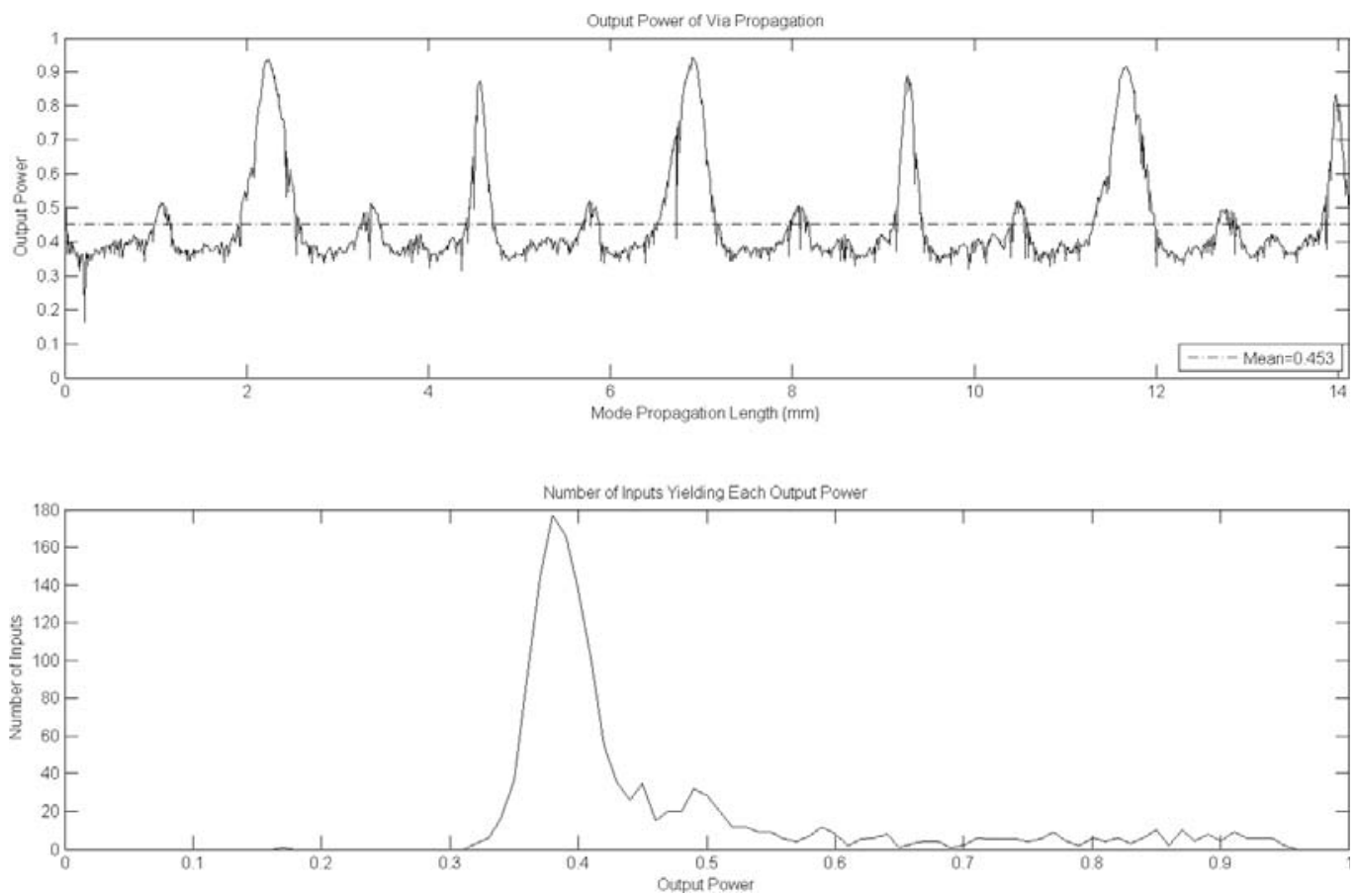

Figure 5.8: Power output after via propagation for $250 \mu \mathrm{m}$ lead-in (off center SM input)
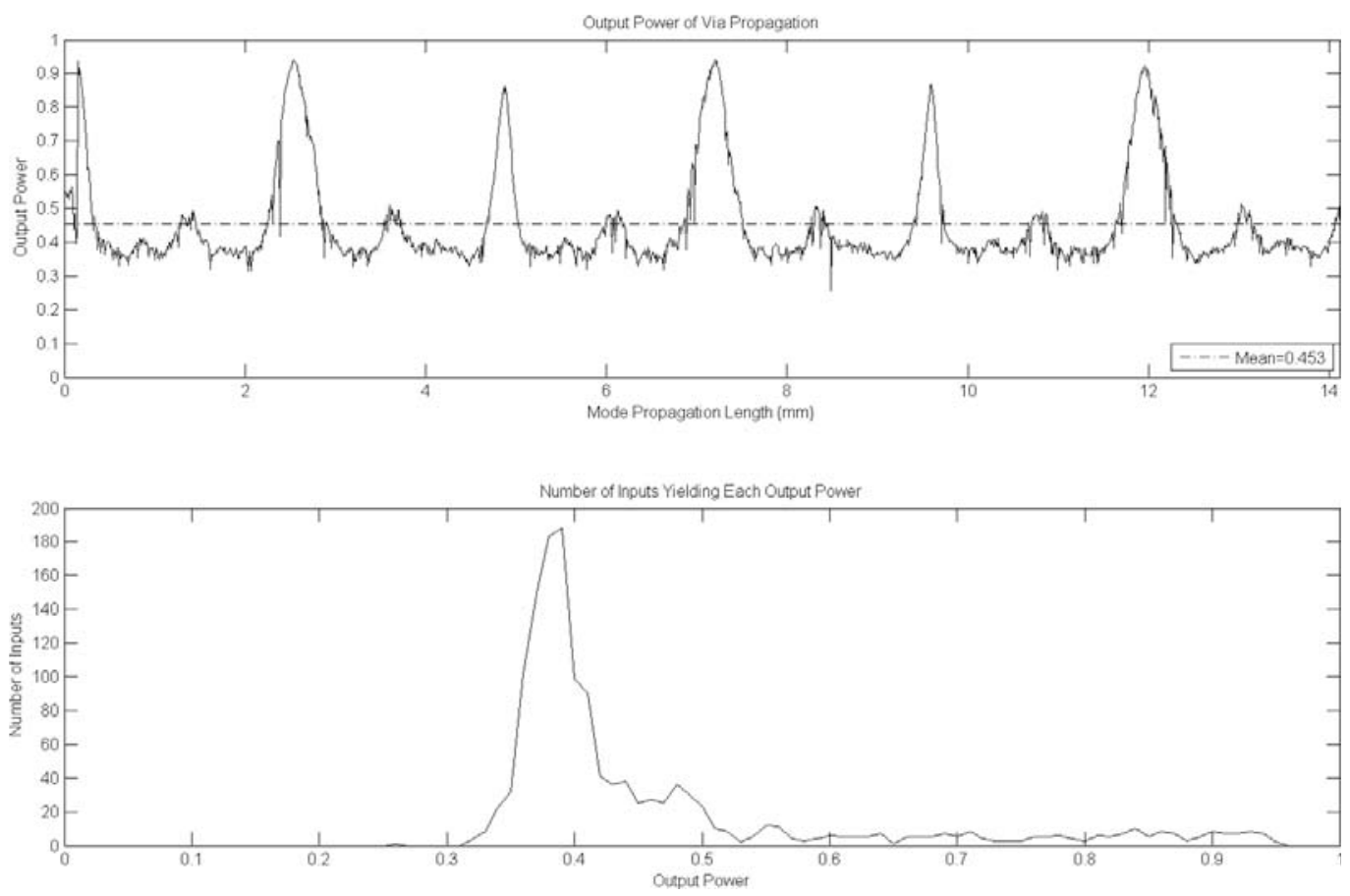

Figure 5.9: Power output after via propagation for $500 \mu \mathrm{m}$ lead-in (off center SM input) 


\subsection{5 mm Straight - Multimode Input}

The multimode input generating function in RSoft fills all supported modes equally and assigns a random phase to each. Some cross sections from the $15 \mathrm{~mm}$ propagation are seen in Figure 5.10 .

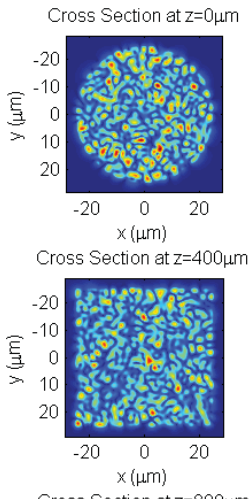

Cross Section at $z=800 \mu \mathrm{m}$

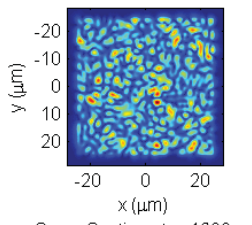

Cross Section at $z=1200 \mu \mathrm{m}$

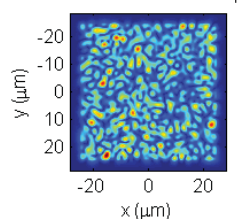

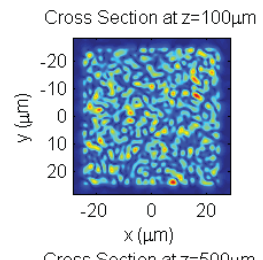

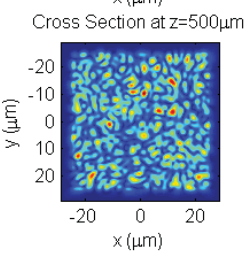

Cross Section at $z=900 \mu \mathrm{m}$
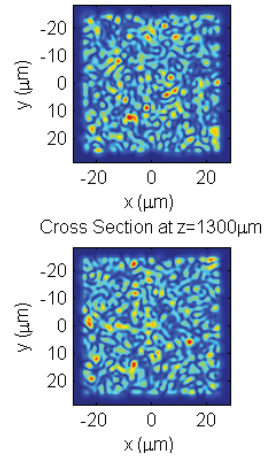
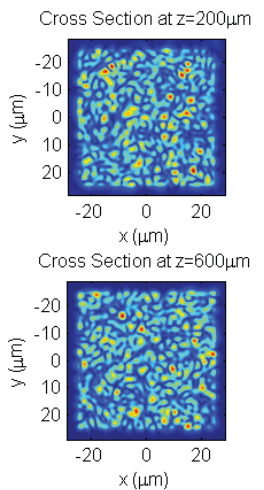

Cross Section at $z=1000 \mu m$

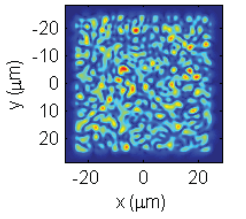

Cross Section at $z=1400 \mu m$

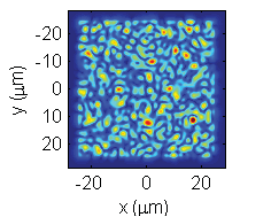

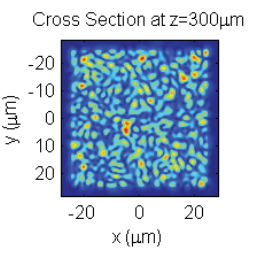

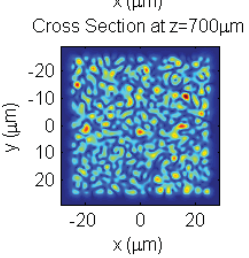

Cross Section at $z=1100 \mu m$

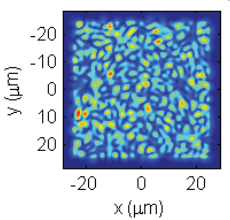

Cross Section at $z=1500 \mu \mathrm{m}$

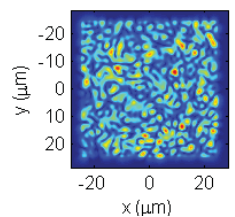

Figure 5.10: Selection of modes for $15 \mathrm{~mm}$ straight with $\mathbf{5 0} \mu \mathrm{m}$ multimode input

Since the multimode input is formed using a random phase assigned to each of the possible modes, it is not expected that the field should repeat itself exactly. As can be seen in Figure 5.11, this is indeed the case. The cross correlation of the fields with the input distribution do not peak to a significant level. Figure 5.12 shows the same information for a different random phase assignment. MM input 1 refers to one particular set of random phase assignments and MM input 2 refers to a different set.

The output data for each set of multimode inputs is seen in Figure 5.13, Figure 5.14, Figure 5.15, and Figure 5.16. Loss levels are at $-4.71,-4.76,-4.67$, and $-4.72 \mathrm{~dB}$, respectively. 
1D Mode Profiles along Propegation Length
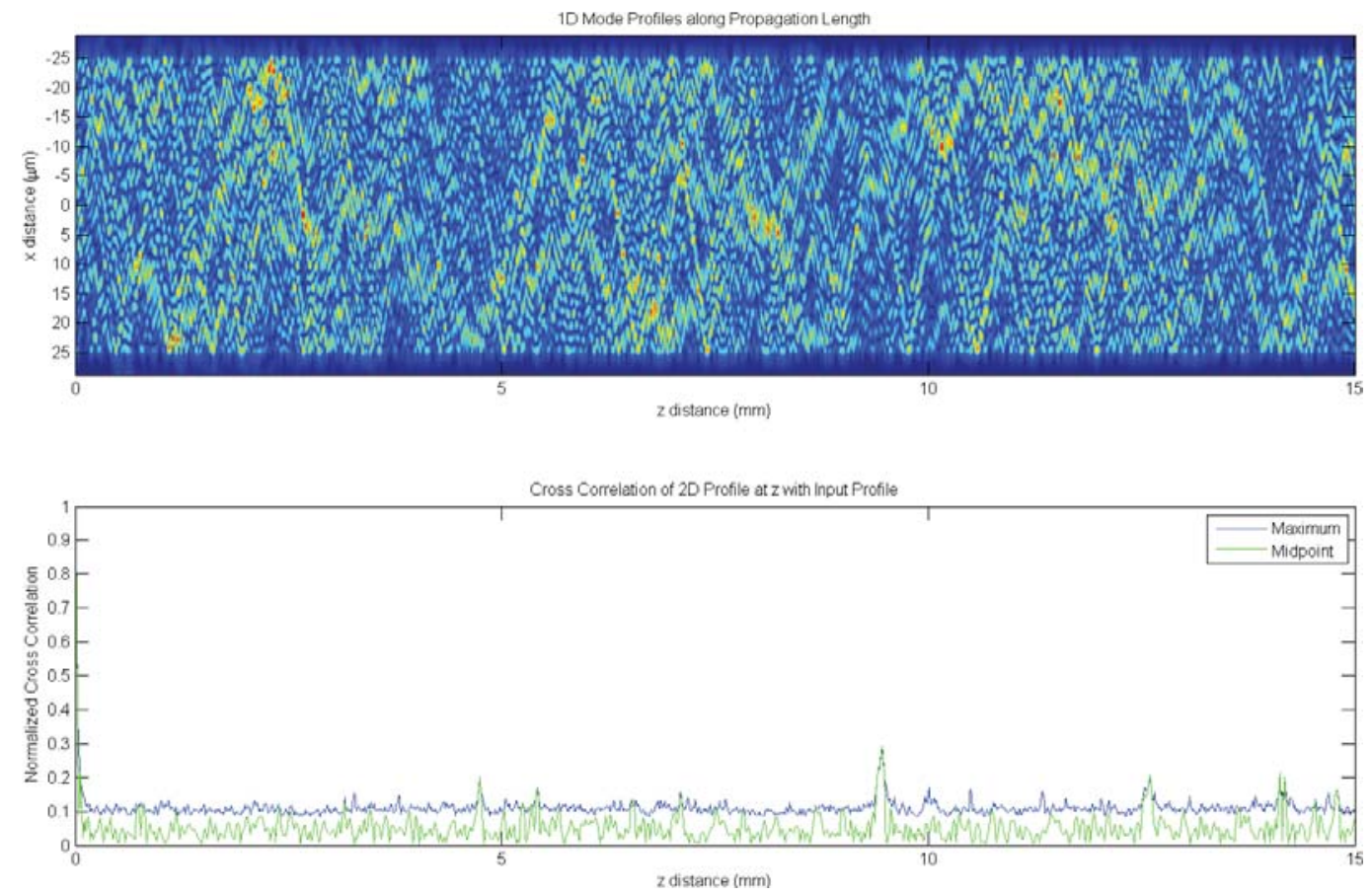

Figure 5.11: $15 \mathrm{~mm}$ straight with multimode input mode profile and cross correlation data (MM input 1)

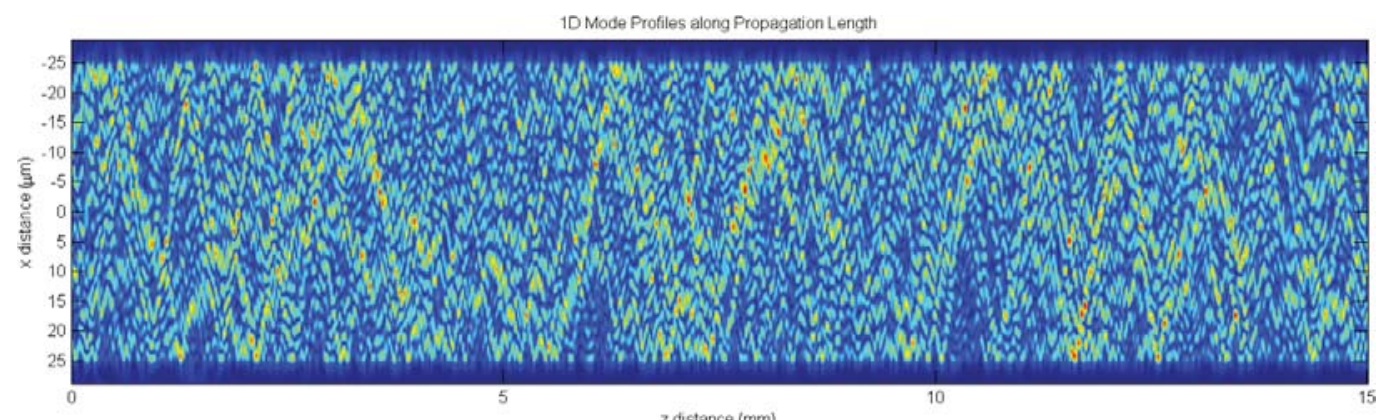

z distance $(\mathrm{mm})$

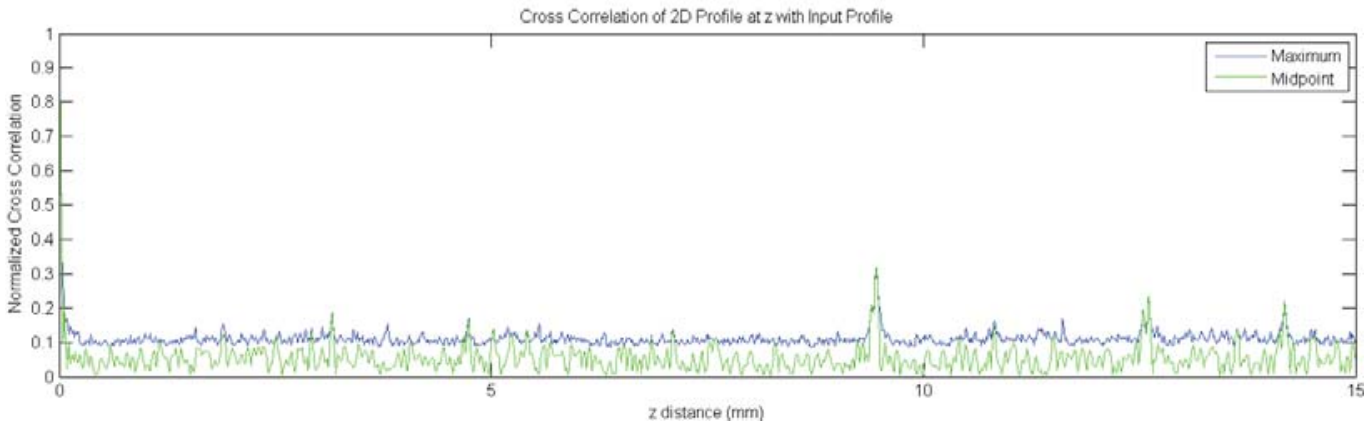

Figure 5.12: $15 \mathrm{~mm}$ straight with multimode input mode profile and cross correlation data (MM input 2) 

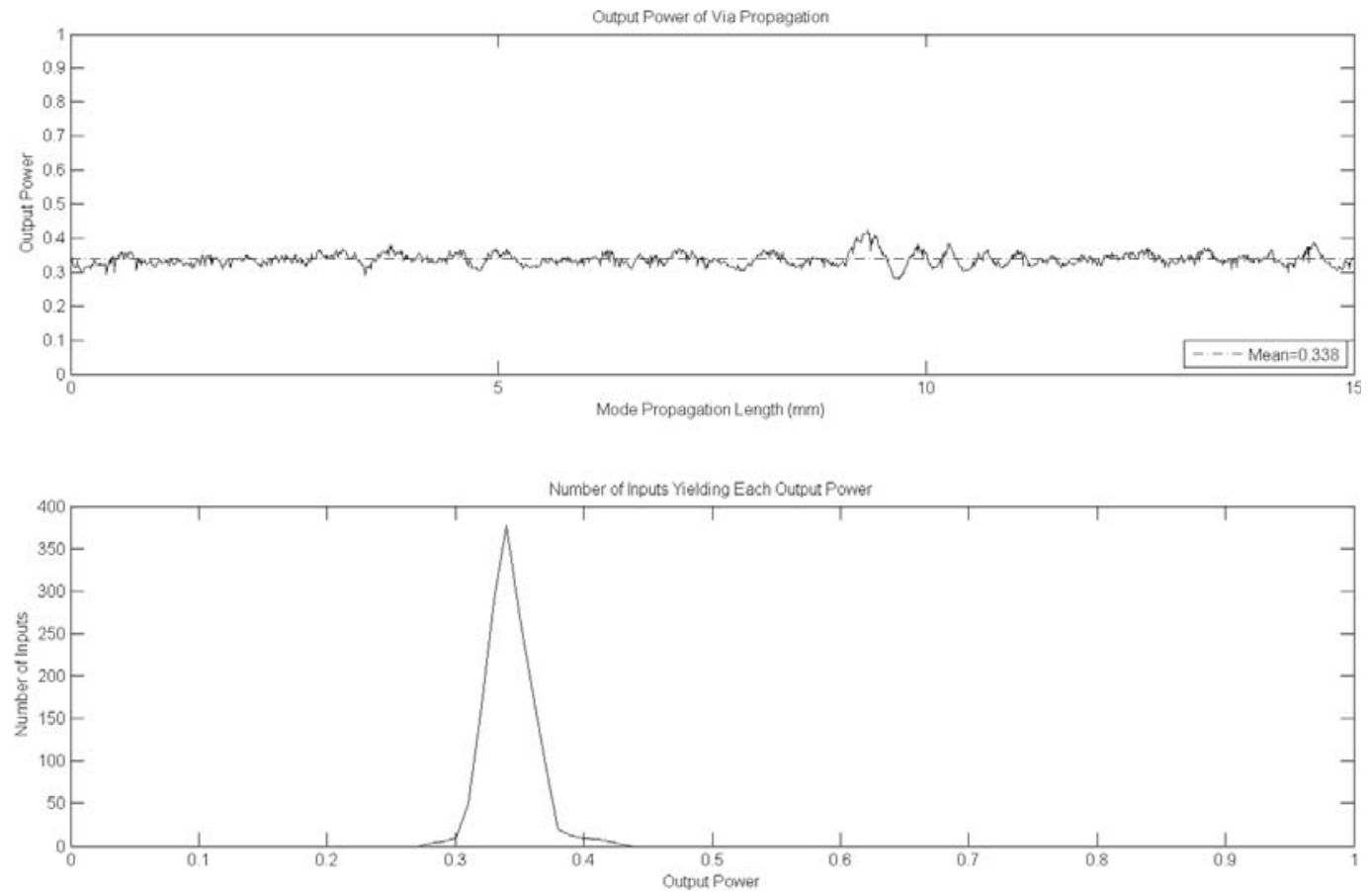

Figure 5.13: Power output after via propagation for $250 \mu \mathrm{m}$ lead-in (MM input 1)
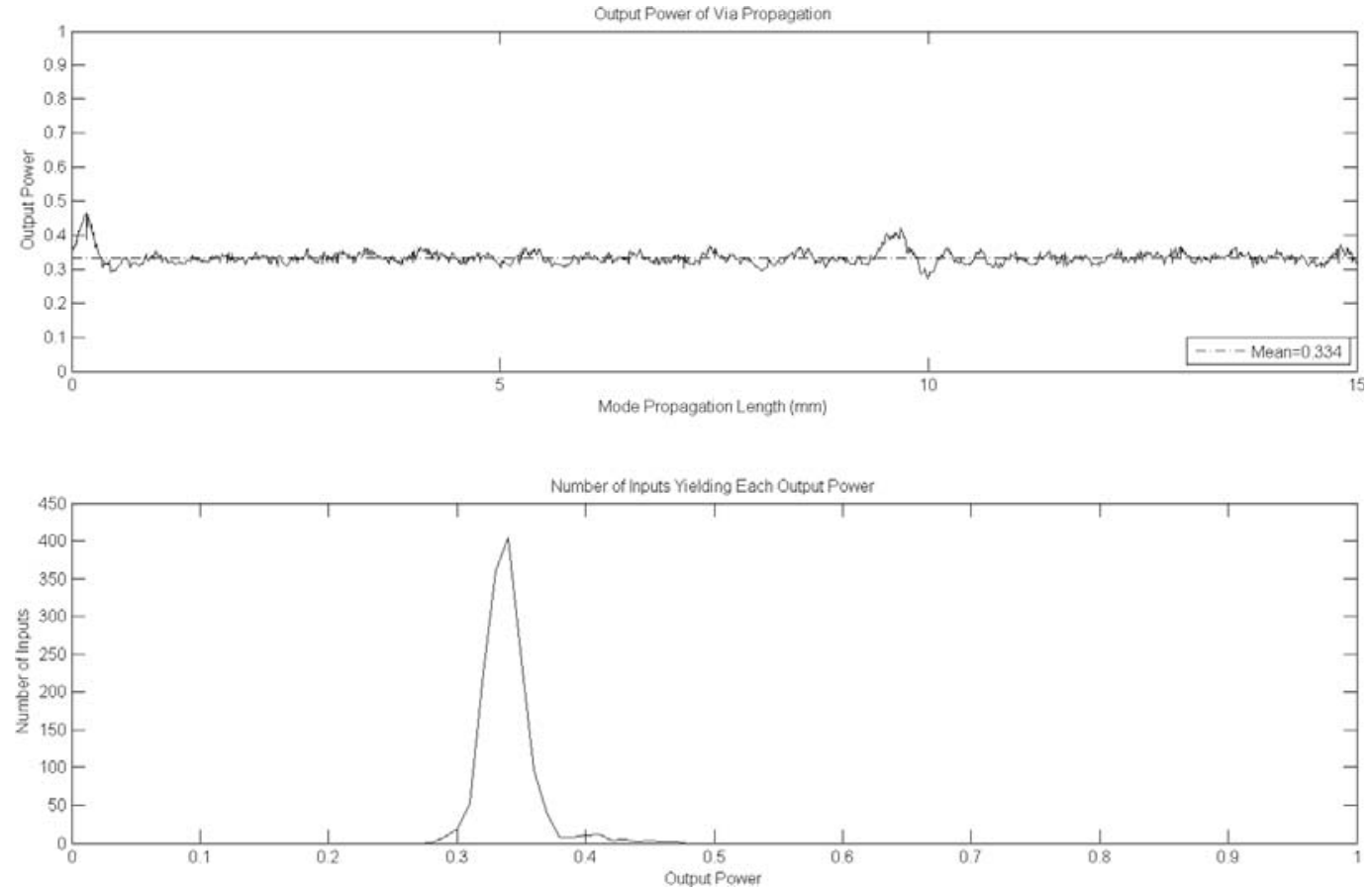

Figure 5.14: Power output after via propagation for $500 \mu \mathrm{m}$ lead-in (MM input 1) 

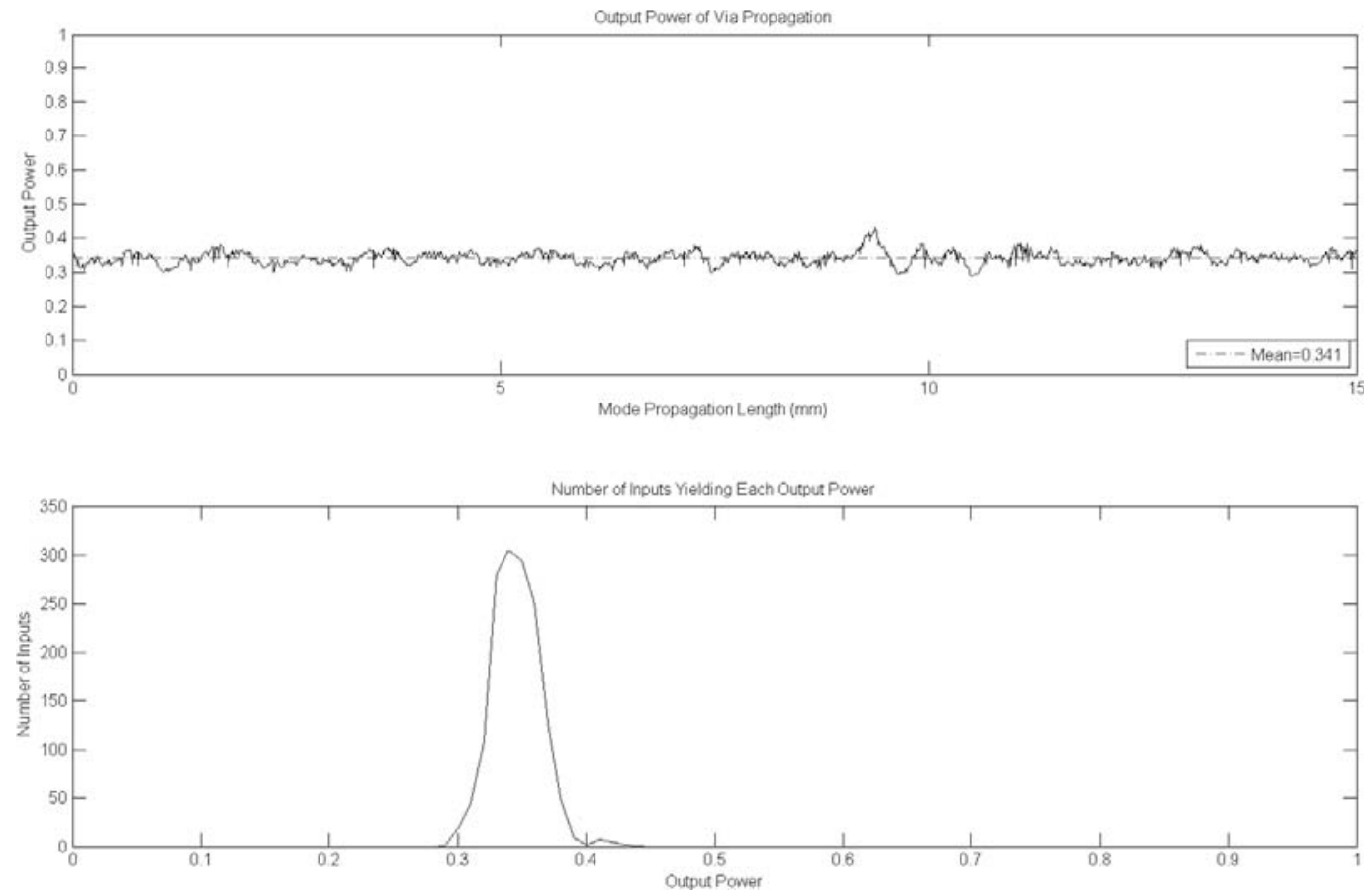

Figure 5.15: Power output after via propagation for $250 \mu \mathrm{m}$ lead-in (MM input 2)
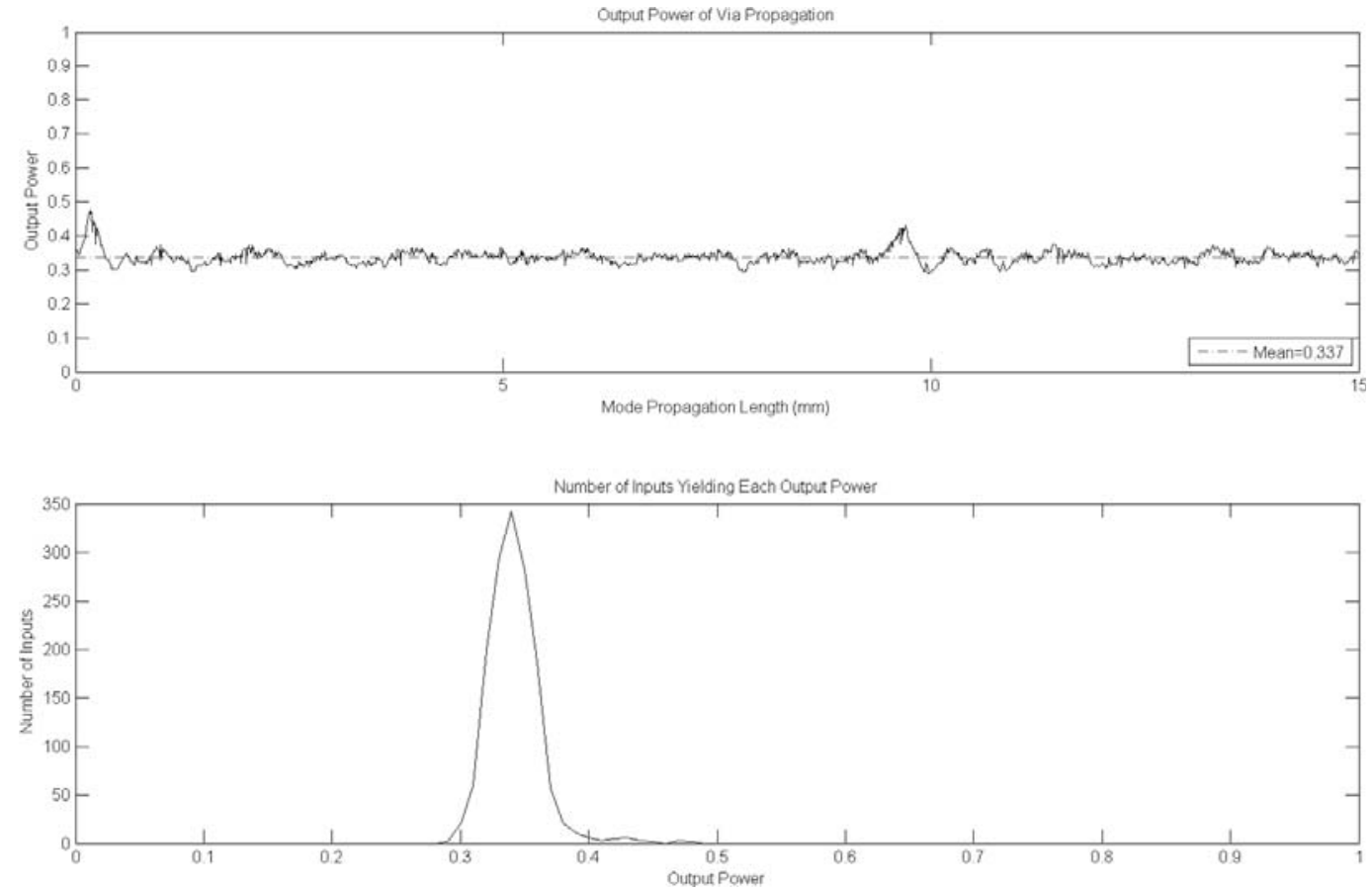

Figure 5.16: Power output after via propagation for $500 \mu \mathrm{m}$ lead-in (MM input 2) 
The propagation simulation was also performed for a lead-in distance of 0 . The results from this simulation are seen in Figure 5.17. Losses at this lead-in distance were $4.88 \mathrm{~dB}$.
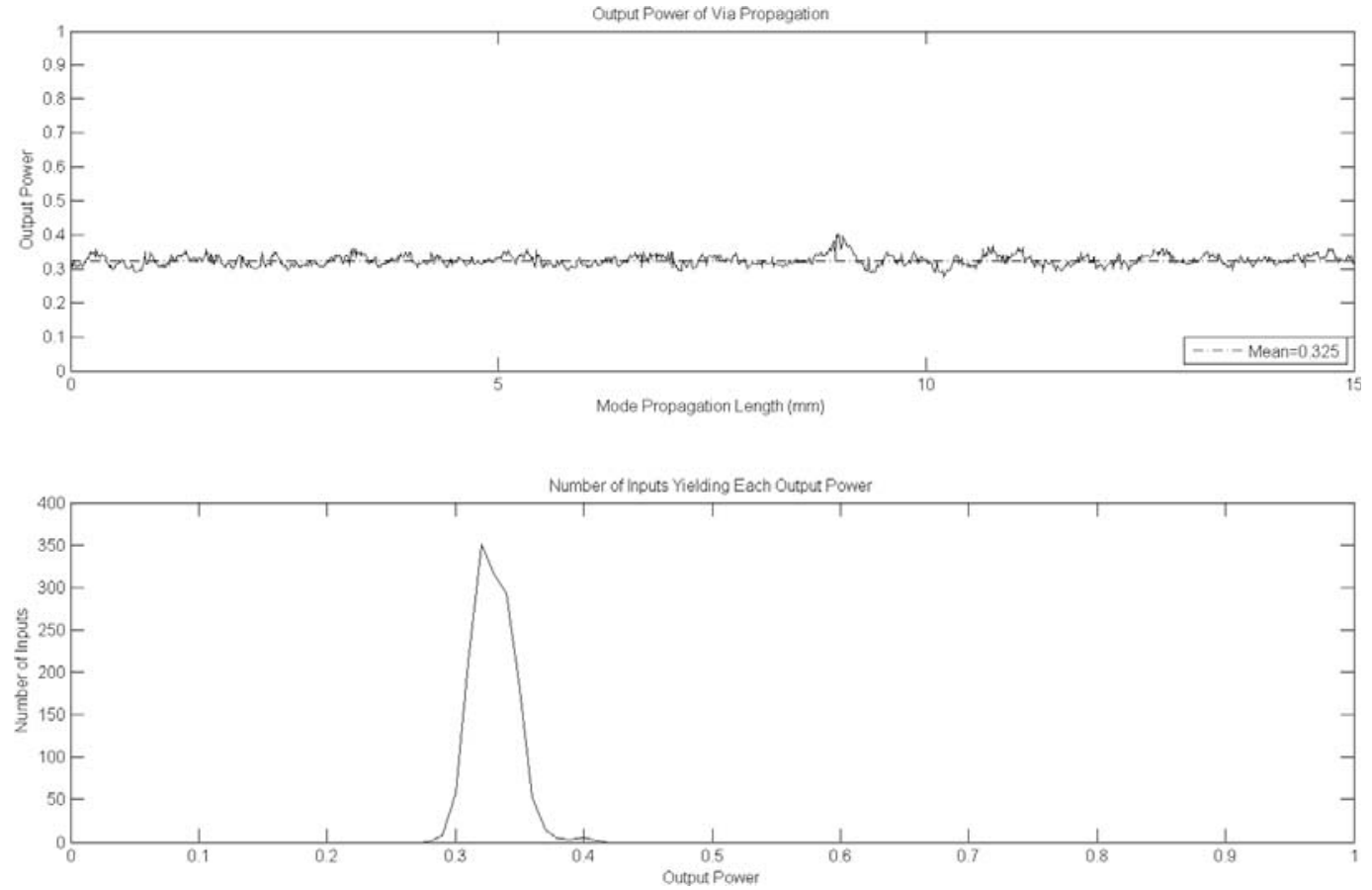

Figure 5.17: Power output after via propagation for $0 \mu \mathrm{m}$ lead-in (MM input 2) 


\subsection{Experimental Data}

These via structures were fabricated and tested by other members of the research group. A $50 \mu \mathrm{m}$ core diameter fiber input was used to the via and output coupling was made using a $62.5 \mu \mathrm{m}$ core diameter fiber. An input power of $1.68 \mathrm{~mW}$ was used. The distance between the end face of the input fiber and the front of the input ball lens was not able to be measured; however this distance was believed to be less than $1 \mathrm{~mm}$.

Table 5.1

Experimental via data at $1.68 \mathrm{~mW}$ input

\begin{tabular}{|c|c|c|c|}
\hline Via & $\begin{array}{c}\text { Output Power } \\
(\mu \mathrm{W})\end{array}$ & $\begin{array}{c}\text { Through Power } \\
(\%)\end{array}$ & $\begin{array}{c}\text { Losses } \\
(\mathrm{dB})\end{array}$ \\
\hline 1 & 180 & 10.71 & 9.70 \\
\hline 2 & 750 & 44.64 & 3.50 \\
\hline 3 & 820 & 48.81 & 3.11 \\
\hline 4 & 550 & 32.74 & 4.85 \\
\hline 5 & 835 & 49.70 & 3.04 \\
\hline 6 & 870 & 51.79 & 2.86 \\
\hline
\end{tabular}

With the exception of via \#1 and \#4, these values fall within the expected range indicated by the simulations. It is possible that a defect was present in the manufacturing of via \#1 or that it was not aligned properly to achieve maximum power throughput of the system. This misalignment is likely the cause of via \#4's low performance. 


\section{Chapter 6}

\section{Conclusion}

\subsection{Discussion of Results}

A multi-software model was proposed for the simulation of light through an optical vertical interconnect assembly. This model took advantage of the integrated optics strengths of RSoft's BeamPROP software and the optical system propagation strength of the ZEMAX optical design software. Waveguide mode structures were generated with the beam propagation method and propagated an optical system using the angular spectrum propagation technique. MATLAB was used to evaluate output data from each step of the process. This included checking for repeating mode structures using the cross correlation of the distributions with the input field so that each field distribution was only used once.

A single mode fiber of core diameter $5.6 \mu \mathrm{m}$ was used as in input to a $15 \mathrm{~mm}$ waveguide to generate these field structures. This type of fiber is what is utilized for the experimental setup in the lab, so it was a natural choice for a simulation input. This single mode input was input to a $50 \mu \mathrm{m}$ square multimode structure. The fiber was placed at the center of the $50 \mu \mathrm{m}$ square as well as in an off center position in the upper right quadrant of the waveguide.

With the centered single mode input, the mode structure was seen to repeat itself at regular intervals. Therefore only the field distributions through the first repetition were propagated through the via model. The highly regular field distributions through this region of the guide showed less than $3 \mathrm{~dB}$ loss through the via. The best performance occurred when most of the power was present in the center of the waveguide.

For the off center single mode input, the mode structure was again seen to repeat itself, but over a longer interval. Again, the field distributions through one repetition of the input were input into the via model. These distributions also showed losses of less than $3 \mathrm{~dB}$. As in the centered single mode input, the mode structures were highly regular with best performance when the majority of the power was present in the middle of the waveguide.

A multimode input was generated in RSoft by uniformly weighting all modes and assigning a random phase to each one. This input method is the most realistic, since boards fabricated with this technology will contain waveguide geometries that will induce mode stirring and many modes will be present. Two different iterations of 
these types of distributions were completed to show the similarity of performance with these two different inputs.

For the multimode inputs, average losses of 3.5 to $4 \mathrm{~dB}$ were seen. These numbers are in agreement with the experimental data that has been gathered on the vias that have been fabricated in the lab.

Higher losses when the input was placed directly on the first surface indicate that there is an optimum distance between 0 and $500 \mu \mathrm{m}$ where the average losses through the via can be minimized.

These results are positive indicators that the link budget of less than $3 \mathrm{~dB}$ per via is achievable. While this particular design does not consistently show this efficiency, it is reasonable to expect that subsequent modifications to the design can improve the overall efficiency within the required range.

The single mode input configurations can be seen to represent a physical configuration where a single mode source is placed above the via and used to insert light into the waveguide network, since the via is a symmetric device and will work the same in both a forward and reverse direction. These simulations as a whole showed lower average losses, but it is even more promising that the mode structures when the field is centered and diverging (similar to the input a VCSEL would provide), losses were lower than the average indicates. While the average indicated just under 3 dB loss, Figure 5.3 and Figure 5.4 show peaks of $100 \%$ transmission ( 0 dB loss).

While input configurations show promising losses of only 0 to $3 \mathrm{~dB}$, output configurations showed greater than $3 \mathrm{~dB}$ losses in most cases. This means that it may be possible that average losses between an input/output pair of vias could be below the $3 \mathrm{~dB}$ target. 


\subsection{Future Work}

The next step for this work would be to develop a model in ZEMAX using the nonsequential mode. In non-sequential mode, the user is allowed to define shapes and volumes of material instead of a sequence of surfaces. This would allow the simulation to account for any guiding effects taking place within the via channels. Another area of interest would be to add an additional step at the end of this process to calculate the coupling efficiency if a fiber were placed at the output of the via. This could be done by using the ZEMAX output as an input into another RSoft step.

Through running this process with different parameters, optimum parameters for the distance between the input waveguide and the first ball lens could be found. This same concept could be applied to the distance between the output ball lens and the surface where power is calculated. Also, as the design of the via changes, these simulations would need to be run again; however the methods are in place to do so. 



\section{References}

[1] N. Bamiedakis, J. Beals IV, R. V. Penty, I. H. White, J. V. DeGroot, T. V. Clapp, "Cost-Effective Multimode Polymer Waveguides for HighSpeed On-Board Optical Interconnects," IEEE Journal of Quantum Electronics, vol. 45, pp. 415-424, 2009.

[2] T. Kurokawa, N. Takato, Y. Katayama, "Polymer optical circuits for multimode optical fiber systems," Applied Optics, vol. 19, pp. 31243129, 1980.

[3] F. Liu, F. Wang, J. Yu, D. Guidotti, A Adibi, G.K. Chang, R. Tummala, M. Moynihan, B. Sicard, "Scaleable Board-Level High Performance Optical Polymer Waveguide Technologies," presented at the 11th International Symposium on Advanced Packaging Materials: Processes, Properties and Interface, Atlanta, Georgia, 2006.

[4] R. M. Gagliardi, Sherman Karp, Optical Communications, 2nd ed. Hoboken, New Jersey: John Wiley \& Sons, Inc., 1995.

[5] D. A. B. Miller, H.M. Ozaktas, "Limit to the Bit-Rate Capacity of Electrical Interconnects from the Aspect Ratio of the System Architecture," Journal of Parallel and Distributed Computing, vol. 41, pp. 42-52, 1997.

[6] B. E. A. Saleh, M. C. Teich, Fundamentals of Photonics, 2nd ed. Hoboken, New Jersey: John Wiley \& Sons, Inc., 2007.

[7] S. Sinzinger, J. Jahns, Microoptics, 2nd ed. Weinheim, Germany: Wiley-VCH GmbH \& Co. KGaA, 2003.

[8] E. Hecht, Optics, 4th ed. San Francisco, California: Addison Wesley, 2002.

[9] M. A. Brook, Silicon in Organic, Organometallic and Polymer Chemistry, 1st ed. New York, New York: John Wiley \& Sons, Inc., 2000.

[10] T. Daunais, K. Walczak, C. Middlebrook, P. Bergstrom, "Characterization of irradiance effects on curing of siloxane for embedded waveguide applications," in Integrated Optics: Devices, Materials, and Technologies XV, 2010.

[11] B. W. Swatowski, C. T. Middlebrook, K. Walczak, M. C. Roggemann, "Optical loss characterization of polymer waveguides on halogen and 
halogen-free FR-4 substrates," presented at the Optoelectronic Interconnects and Component Integration XI, 2011.

[12] P. N. Butcher, D. Cotter, The Elements of Nonlinear Optics, Digital ed. Cambridge, UK: Cambridge University Press, 2003.

[13] R. Scarmozzino, A. Gopinath, R. Pregla, "Numerical Techniques for Modeling Guided-Wave Photonic Devices," IEEE Journal of Selected Topics in Quantum Electronics, vol. 6, pp. 150-162, 2000.

[14] E. W. Weisstein. Padé Approximant. MathWorld - A Wolfram Web Resource. Available: http://mathworld.wolfram.com/PadeApproximant.html

[15] G. R. Hadley, "Wide-angle beam propagation using Padé approximant operators," Optics Letters, vol. 17, pp. 1426-1428, October 15, 1992 1992.

[16] K. S. Yee, "Numerical Solution of Initial Boundary Value Problems Involving Maxwell's Equations in Isotropic Media," IEEE Transactions on Antennas and Propagation, vol. AP-14, pp. 302-307, 1966.

[17] ZEMAX Optical Design Program User's Guide: ZEMAX Development Corporation, 2008.

[18] J. W. Goodman, Introduction to Fourier Optics, 3rd ed. Greenwood Village, Colorado: Roberts \& Company Publishers, 2005.

[19] R. C. Gonzalez, R. E. Woods, Digital Image Processing, 3rd ed. Upper Saddle River, New Jersey: Pearson Education, Inc., 2008.

[20] BeamPROP User Guide, Revision 3 ed.: RSoft Design Group, Inc., 2010.

[21] J. Van Roey, J. van der Donk, P. E. Lagasse, "Beam-propagation method: analysis and assessment," Journal of the Optical Society of America, vol. 71, pp. 803-810, 1981.

[22] P.-L. Fan, M.L. Wu, C.T. Lee, "Analysis of Abrupt Bent Waveguides by the Beam Propagation Method and Conformal Mapping Method," Journal of Lightwave Technology, vol. 15, pp. 1026-1031, 1997.

[23] W. Buller, C. Middlebrook, N. Riegel, M. Roggemann, "Methods for Modeling Multimode Waveguides with Abrupt Changes in Propagation Axis," presented at the Avionics, Fiber-Optics and Photonics 2010, Denver, Colorado, 2010. 


\section{Appendix}

\section{MATLAB Code READZEMAX Function}

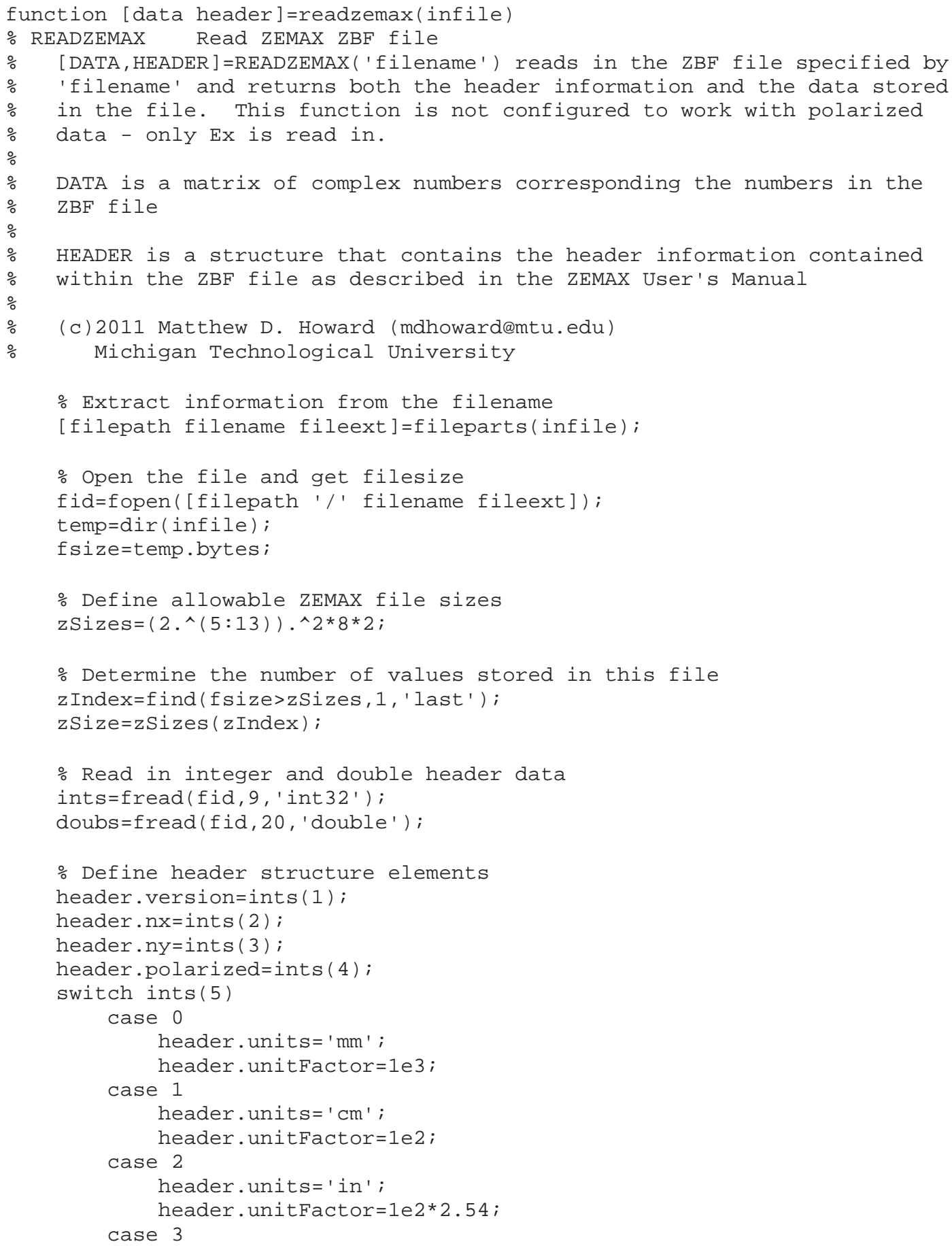




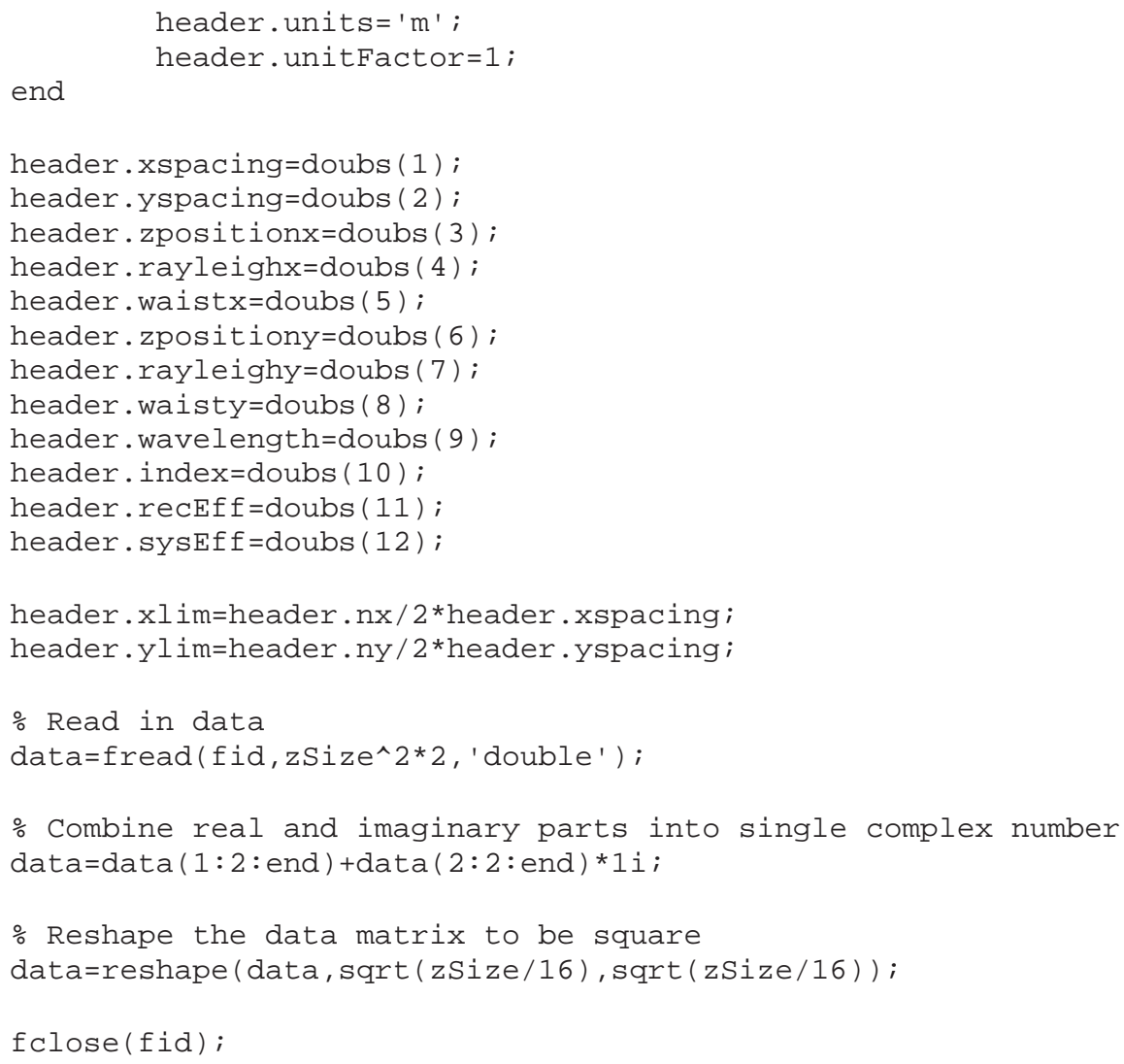

\section{Input Mode Analysis Script}

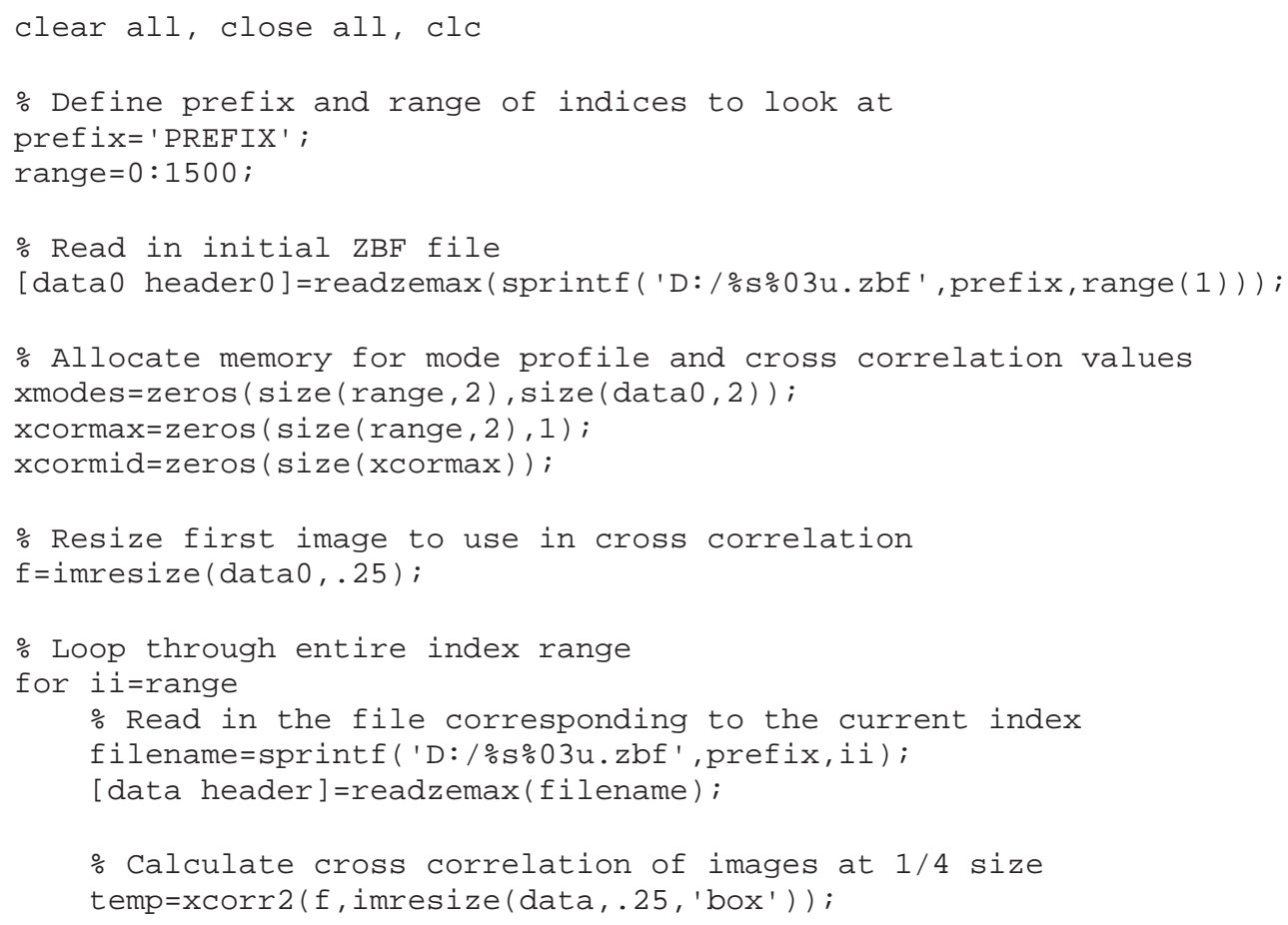




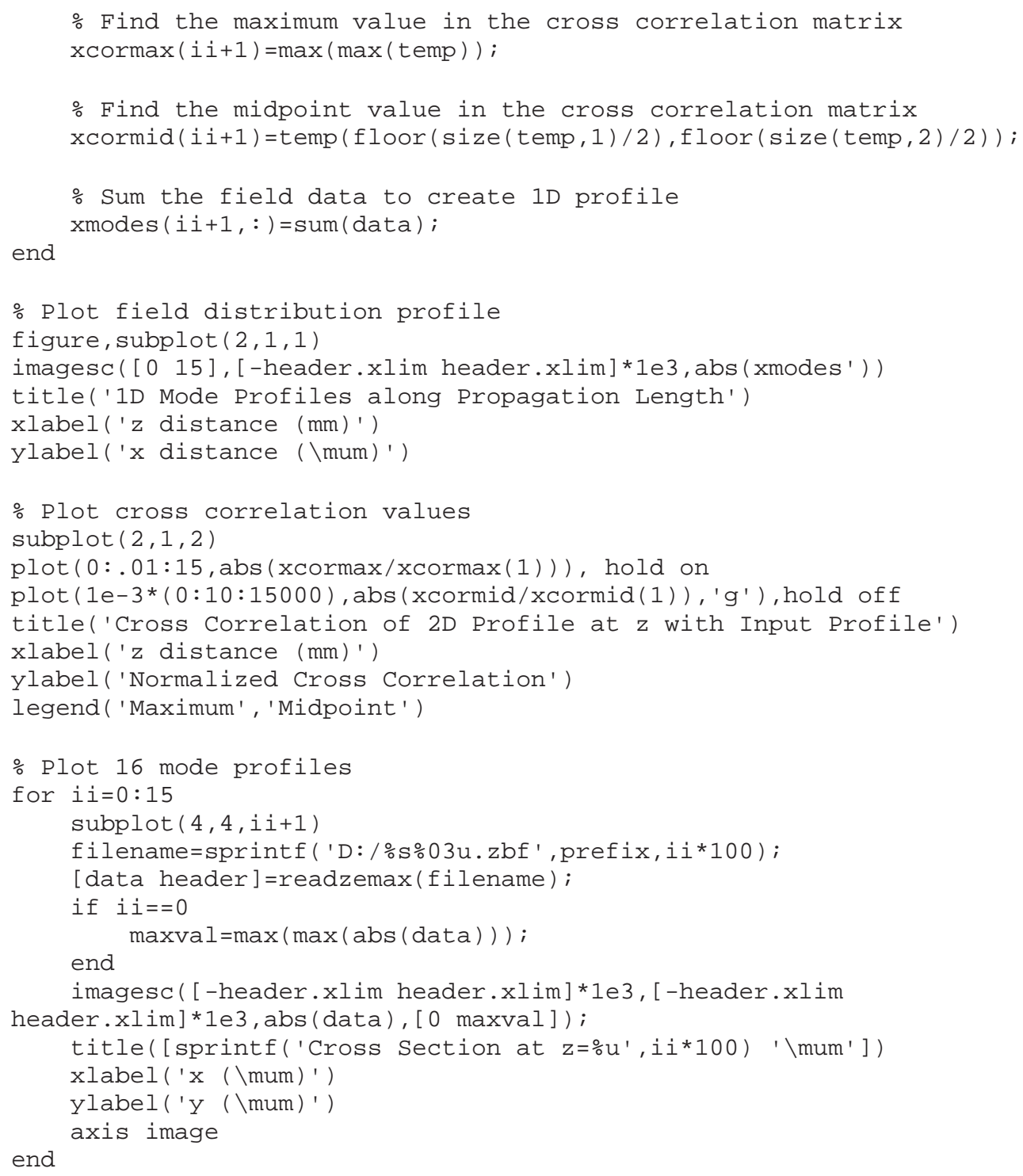

\section{Output Analysis Script}

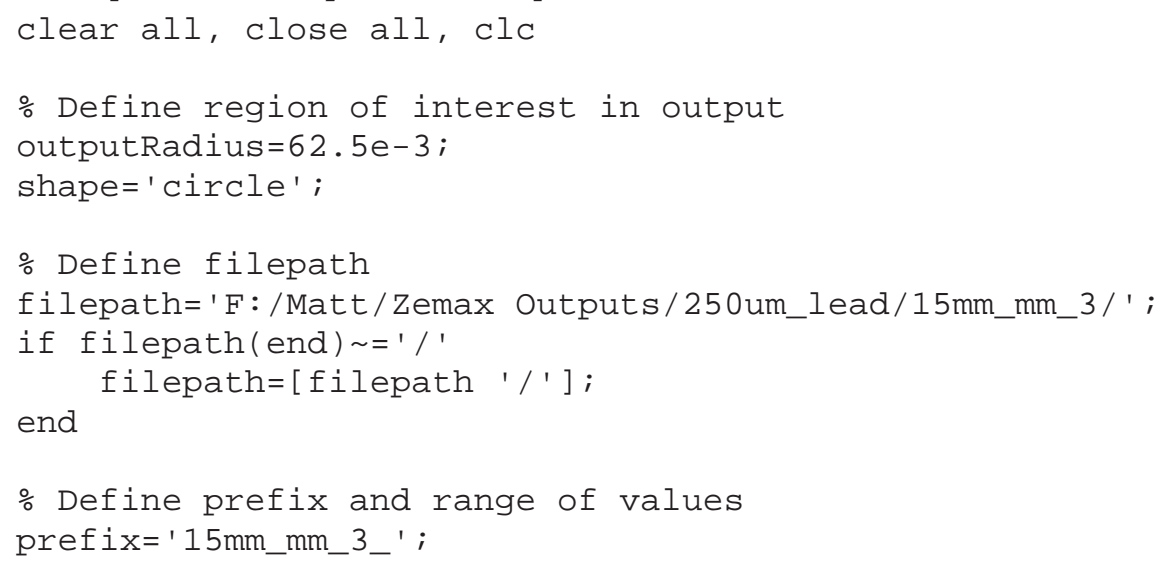




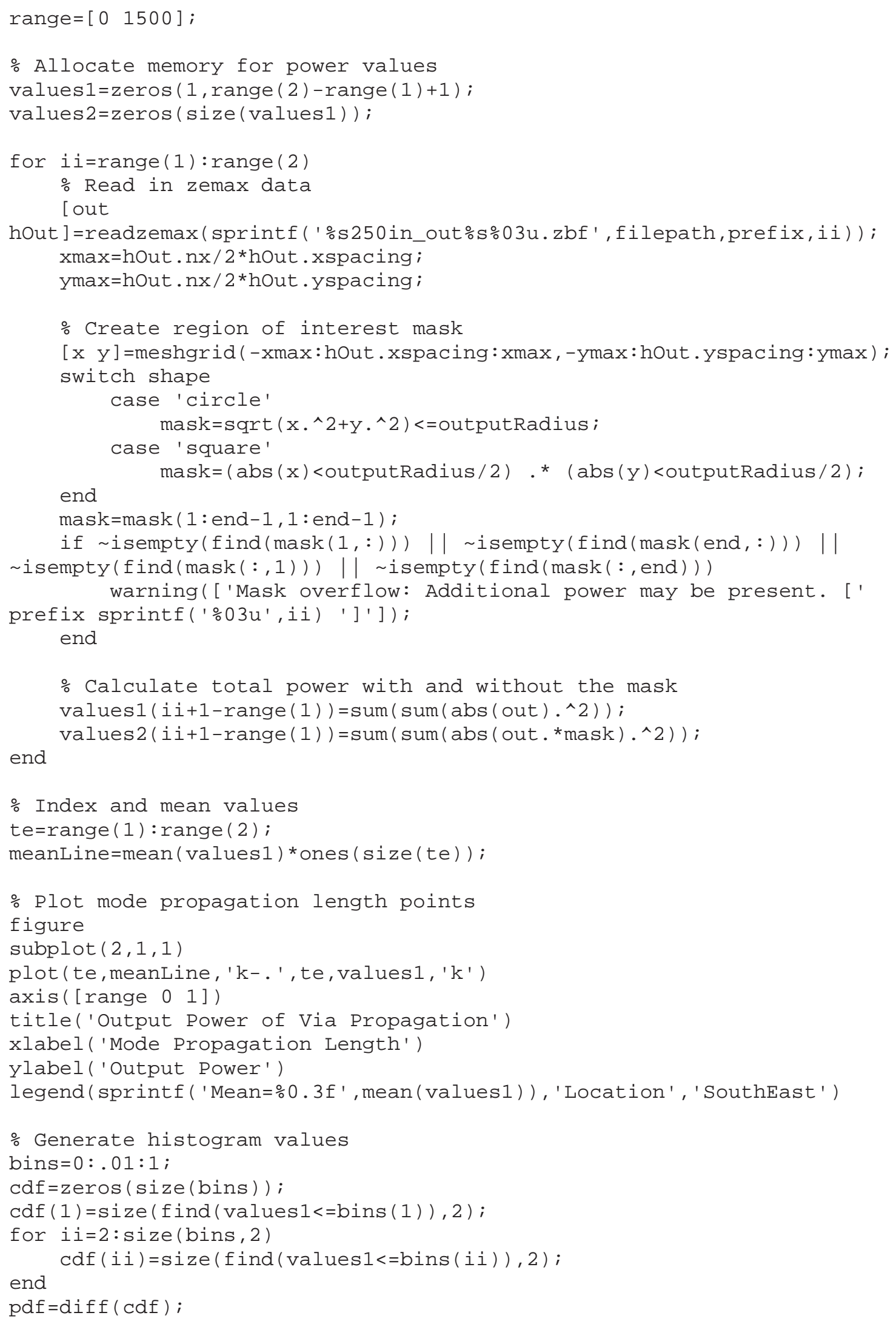




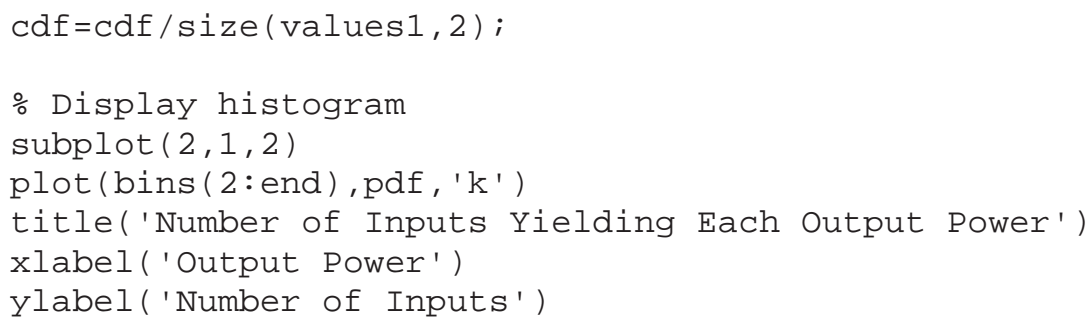

\section{ZEMAX ZPL Code}

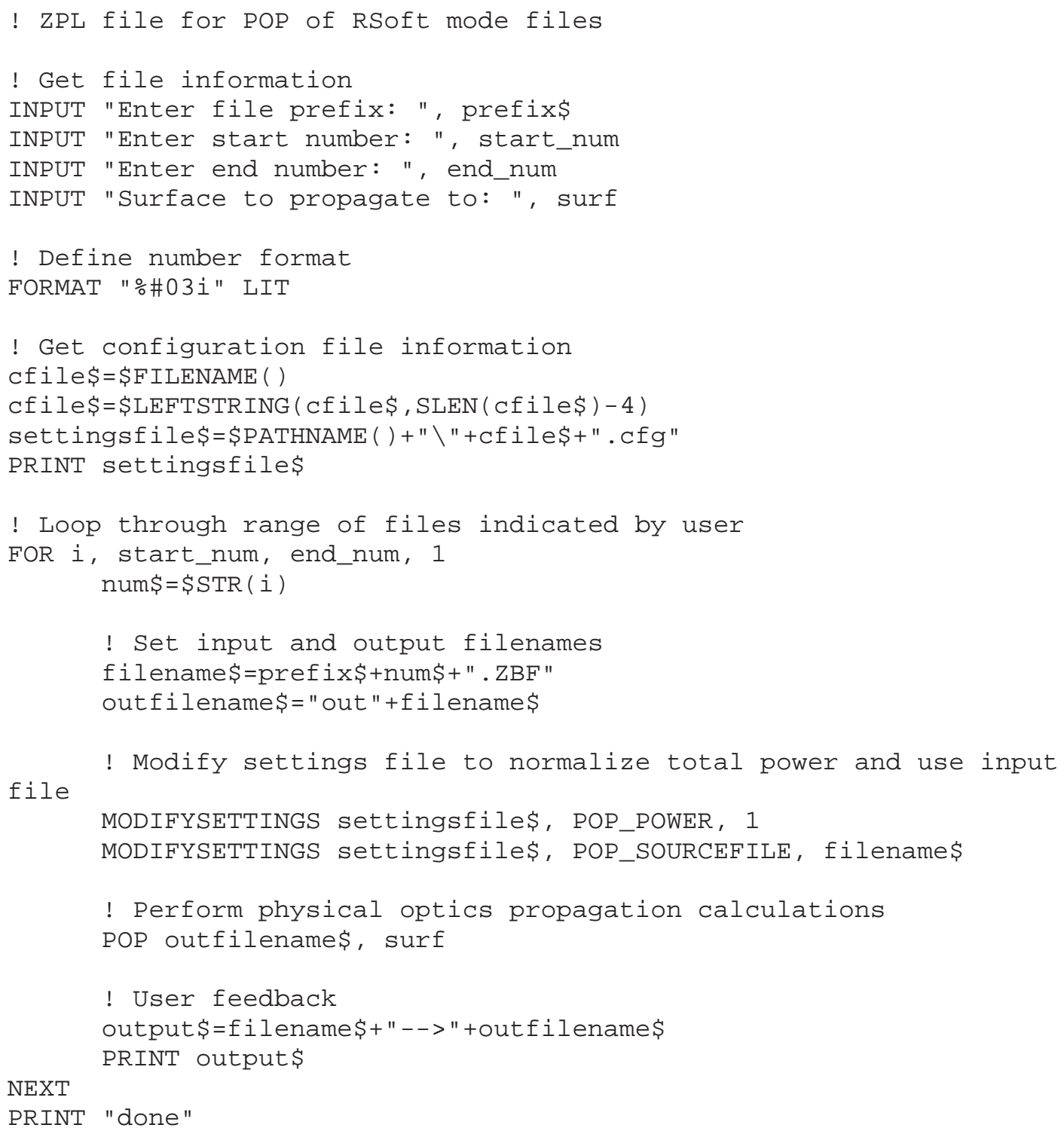




\section{RSoft FLD2ZBF Instructions}

RSoft (".fld" field file) $\leftrightarrow$ Zemax (".zbf" beamfile) Converter

This converter consists of 2 command-line executables:

"fld2zbf.exe" which converts ".fld" files to ".zbf" files (RSoft to Zemax)

"zbf2fld.exe" which converts ".zbf" files to ".fld" files (Zemax to RSoft)

fld2zbf.exe:

The command-line syntax for this executable is:

$>$ fld2zbf [-options] <Ex file> [<Ey file> $]<$ output prefix $>$

options:

Z\# $\quad$ Z position relative to the pilot beam waist $(\mathrm{mm})$

R\# Rayleigh distance for the pilot beam $(\mathrm{mm})$

L\# Wavelength $(\mathrm{mm})$

W\# Waist $(\mathrm{mm})$ of the pilot beam

E\# Receiver efficiency

S\# System efficiency

P\# Set power (Watts)

? $\quad$ prints help

Ex file:

RSoft 3D file (for $x$ polarization of the field) (any suffix will do, ".fld" is an example)

Ey file (optional):

RSoft 3D file (for y polarization of the field) (any suffix will do, ".fld" is an example)

output prefix:

prefix for resulting ".zbf"

Comments:

The $Z, R, L$, \& W options should be provided by the user, since BeamPROP does not calculate these. Some calculation in Zemax will be undefined if these are left as default.

The $Z, R, L, W, E, \& S$ options are just passed through the converter to Zemax The Ey input file is optional, if present, the Beam File will be polarized

The user can set the beam power with the -P option (this essentially renormalized the field)

fld2zbf.exe:

The command-line syntax for this executable is:

> zbf2fld [-options] <input-file-name> <output-file-prefix> 
options:

? $\quad$ prints help

input-file-name:

Zemax ".zbf" file

output-file-prefix:

prefix for resulting ".fld" file(s)

Comments:

If the beamfile is polarized, then ".Ex" \& ".Ey" files will be produced, otherwise, just an ".Ex" file will result (same format as typical ".fld" file)

A plot script ".pcs" is also produced 